Aus der Klinik für Anästhesiologie

(Prof. Dr. med. K. Meissner)

der Medizinischen Fakultät der Universität Göttingen

\title{
Der Einfluss chronischer Schmerzen als Komorbidität auf den klinischen Verlauf bei Patienten mit akuter Herpes-Zoster-Infektion
}

\author{
INAUGURAL - DISSERTATION \\ zur Erlangung des Doktorgrades \\ der Medizinischen Fakultät der \\ Georg-August-Universität zu Göttingen
}

vorgelegt von

Felix Hermann Brandebusemeyer

aus

Mettingen

Göttingen 2019 
Dekan:

Referent:

Ko-Referent/in:

Drittreferent/in:

Datum der mündlichen Prüfung:
Prof. Dr. med. W. Brück

Prof. Dr. med. F. Petzke

Prof. Dr. med. J. Frank

Prof. Dr. med. M. Oppermann

29.06.2021 
Hiermit erkläre ich, die Dissertation mit dem Titel „Der Einfluss chronischer Schmerzen als Komorbidität auf den klinischen Verlauf bei Patienten mit akuter Herpes-Zoster-Infektion“ eigenständig angefertigt und keine anderen als die von mir angegebenen Quellen und Hilfsmittel verwendet zu haben.

Ort, Datum

Unterschrift 
Die Daten, auf denen die vorliegende Arbeit basiert, wurden teilweise publiziert:

Erlenwein J, Thoms K-M, Brandebusemeyer F, Pfingsten M, Mansur A, Quintel M, Schön MP, Petzke F (2016): Pre-Existing Chronic Pain Influences the Severity of Acute Herpes Zoster Pain-A Prospective Observational Cohort Study. Pain Med 17, 1317-1328 


\section{Inhaltsverzeichnis}

Abbildungsverzeichnis.....................................................II

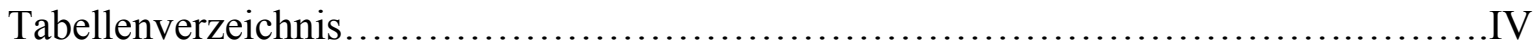

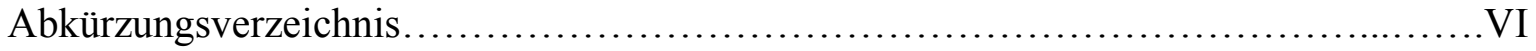

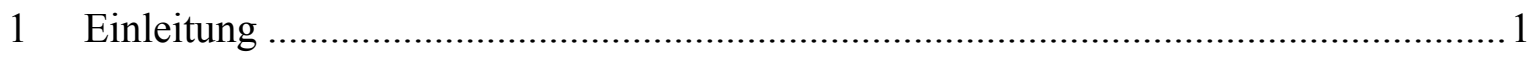

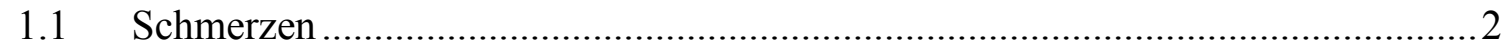

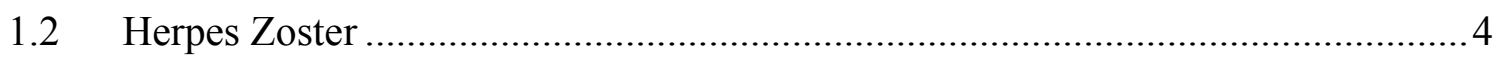

1.3 Schmerzen bei Herpes-Zoster-Infektionen ....................................................... 5

1.4 Therapieoptionen des akuten Herpes Zoster ....................................................... 7

1.5 Prävention und Therapie der Post-Zoster-Neuralgie........................................ 8

1.6 Prädiktoren für akute und chronische postoperative Schmerzen ......................... 9

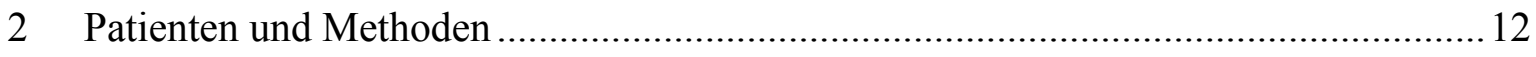

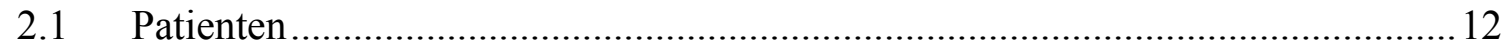

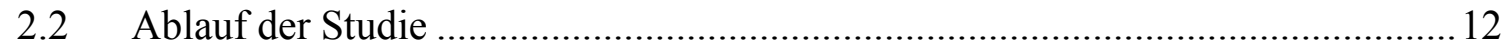

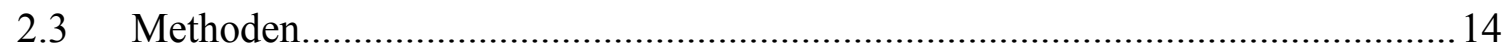

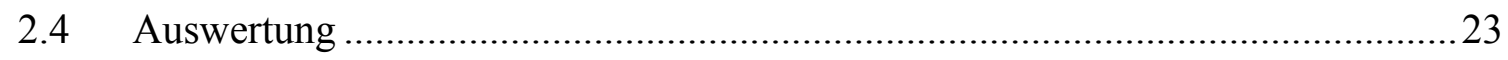

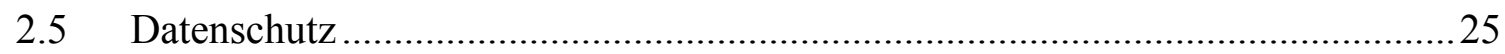

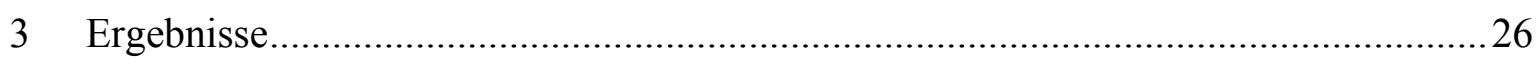

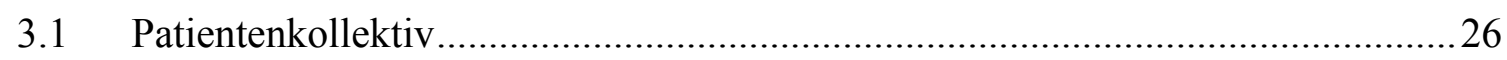

3.2 Vergleich der Patienten mit und ohne vorbestehende chronische Schmerzen...... 32

3.3 Einflussfaktoren auf die Intensität des akuten Zosterschmerzes.........................41

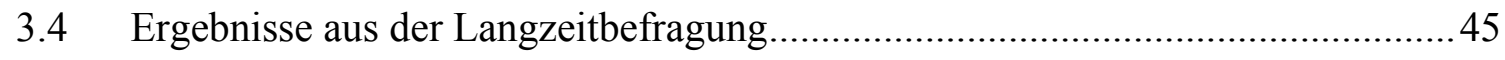

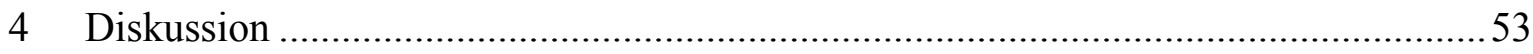

4.1 Patienten mit vorbestehenden chronischen Schmerzen ....................................53

4.2 Risikoindikatoren für ungünstige Krankheitsverläufe …....................................56

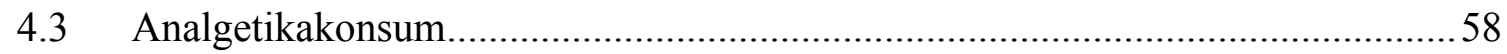




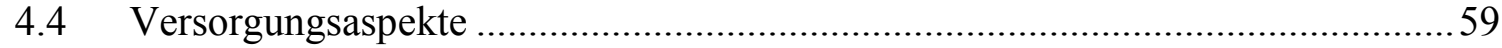

4.5 Definition der chronischen Schmerzen und der Post-Zoster-Neuralgie ...............60

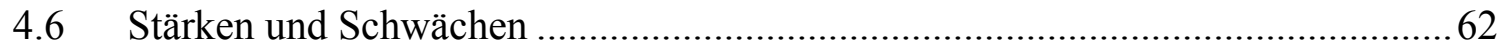

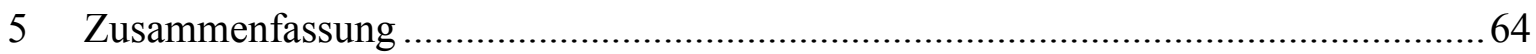

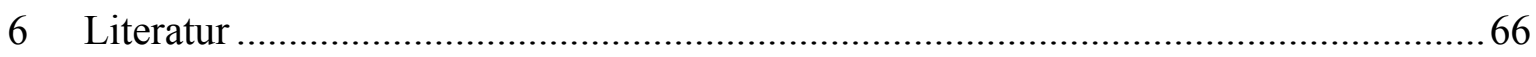




\section{Abbildungsverzeichnis}

Abbildung 1 Flow Chart des Studienablaufs

Abbildung 2 Absolute Anzahl der Schmerzlokalisationen bei Patienten mit chronischen

Schmerzen

Abbildung 3 Verteilung der Chronic Pain Grade nach von Korff bei den Patienten mit

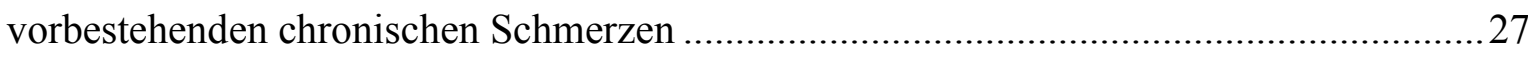

Abbildung 4 Betroffene Dermatome der Herpes-Zoster-Infektion ................................28

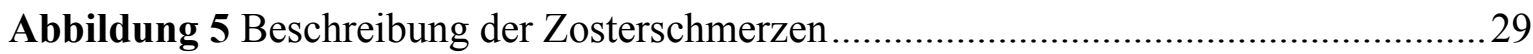

Abbildung 6 Maximale Intensität der Zosterschmerzen im Verlauf der stationären

Behandlung 30

Abbildung 7 Schmerzmedikation (MQS) im Verlauf der stationären Behandlung 30

Abbildung 8 Somatisierungstendenz (PHQ)

Abbildung 9 Verteilung der Geschlechter in den Gruppen nCP und CP 32

Abbildung 10 Körperliche und psychische Lebensqualität (SF 12) 33

Abbildung 11 Depression, Angst und Stress während der stationären Behandlung 34

Abbildung 12 Schmerzverarbeitungsmuster (KPI)

Abbildung 13 Somatisierungstendenz (PHQ) am Aufnahme- und Entlassungstag 35

Abbildung 14 Intensität der Zosterschmerzen im Verlauf. 37

Abbildung 15 Analgetikakonsum (MQS Gesamtscore)

Abbildung 16 Mittelwert und Standardabweichung der Druckschmerzschwelle bei den Patienten mit und ohne vorbestehende chronische Schmerzen

Abbildung 17 Betroffene Dermatome der PZN.

Abbildung 18 Lebensqualität (SF 12), 6 Monate nach der Entlassung 47 


\section{Tabellenverzeichnis}

Tabelle 1 Definitionen der Post-Zoster-Neuralgie ...................................................... 6

Tabelle 2 Medikamentöse Therapieoptionen der Post-Zoster-Neuralgie ............................ 9

Tabelle 3 Prädiktoren für starke postoperative Akutschmerzen .................................... 10

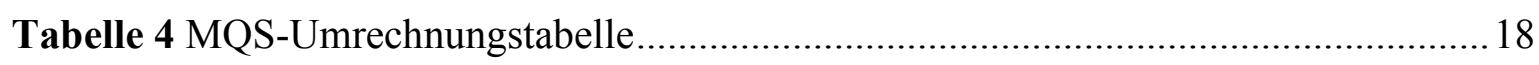

Tabelle 5 Übersicht der erhobenen Parameter während der Studie .................................22

Tabelle 6 Zusammenhang der gemessenen Schmerzintensitäten zu verschiedenen

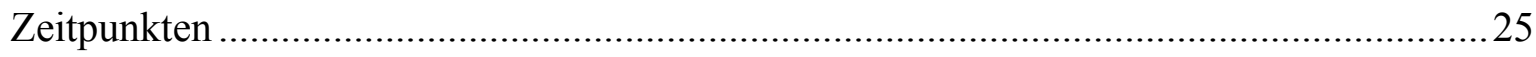

Tabelle 7 Schulbildung, Ausbildung und aktuelle Tätigkeit der Patienten.......................26

Tabelle 8 Psychologische Charakteristika für den Aufnahmetag ..................................... 32

Tabelle 9 Depression, Angst und Stress zum Aufnahmezeitpunkt .................................. 34

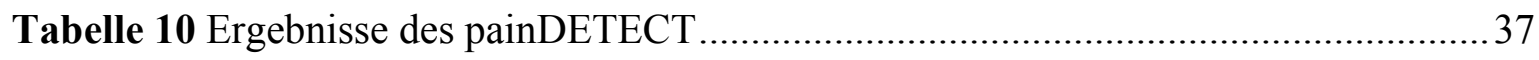

Tabelle 11 Beeinträchtigungen während des stationären Aufenthalts ............................... 38

Tabelle 12 Analgetikakonsum während der stationären Behandlung ............................. 40

Tabelle 13 Zufriedenheit mit der Schmerztherapie (NRS 0-15) .................................... 40

Tabelle 14 Univariate Analyse: Korrelation zwischen den Parametern und der maximalen

Schmerzintensität bei Aufnahme, Tag 4 und Tag 7 / Entlassung .................................... 42

Tabelle 15 Unabhängige Prädiktoren für die maximale Schmerzintensität bei Aufnahme,

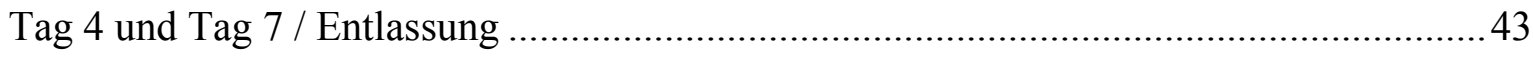

Tabelle 16 Ergebnisse der univariaten Analyse ......................................................... 44

Tabelle 17 Ergebnisse der multivariaten Analyse................................................... 45

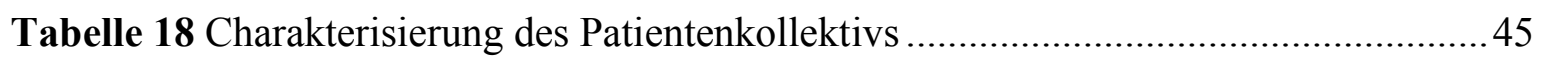

Tabelle 19 Stammdaten der Patienten mit und ohne einer Post-Zoster-Neuralgie .............46 
Tabelle 20 Analgetikakonsum der PZN- und nPZN-Patienten, 6 Monate nach der Entlassung...... 48

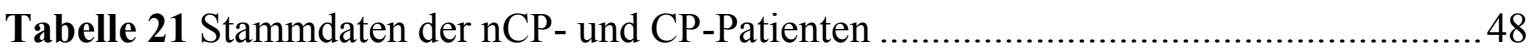

Tabelle 22 Vergleich der Zoster Schmerzintensität zwischen nCP- und CP-Patienten......49

Tabelle 23 Verteilung der Patienten mit und ohne vorstehende chronische Schmerzen in

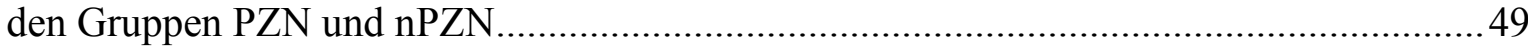

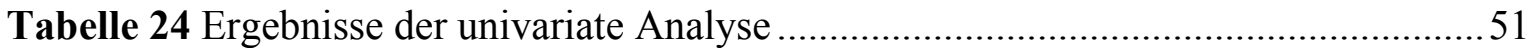

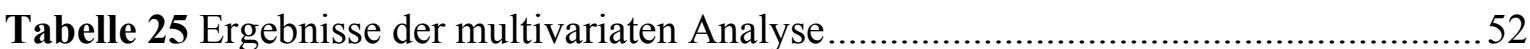

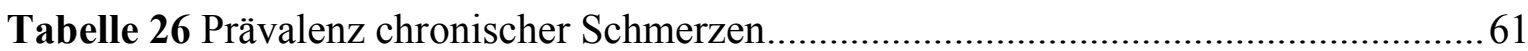




\section{Abkürzungsverzeichnis}

\begin{tabular}{|c|c|}
\hline $3 \mathrm{M}$ & 3 Monate \\
\hline $6 \mathrm{M}$ & 6 Monate \\
\hline BMI & Body Mass Index \\
\hline $\mathrm{CP}$ & Chronic Pain \\
\hline $\mathrm{CPG}$ & Chronic Pain Grade \\
\hline DASS & Depression, Anxiety and Stress Scale \\
\hline DSF & Deutscher Schmerzfragebogen \\
\hline IASP & International Association for the Study of Pain \\
\hline KG & Körpergewicht \\
\hline KPI & Kieler Pain Inventory \\
\hline KSK & Körperliche Summenskala (SF 12) \\
\hline $\mathrm{m}$ & Männlich \\
\hline $\mathrm{ME}$ & Morphinäquivalent \\
\hline MPSS & Mainz Pain Staging System \\
\hline MQS & Medication Quantificaton Score \\
\hline $\mathrm{nCP}$ & Non-Chronic Pain \\
\hline$n P Z N$ & Gruppe der Patienten ohne Post-Zoster-Neuralgie \\
\hline NRS & Numerische Rating Skala \\
\hline n.s. & Nicht signifikant \\
\hline NSAR & Nichtsteroidales Antirheumatikum \\
\hline p.o. & Per os \\
\hline PHQ & Patient Health Questionnaire \\
\hline PSK & Physische Summenskala (SF 12) \\
\hline $\mathrm{PZN}$ & Post-Zoster-Neuralgie / Gruppe der Patienten mit PZN \\
\hline QUIPS & Qualitätsverbesserung in der postoperativen Schmerztherapie \\
\hline SF 12 & Short-Form 12 \\
\hline STIKO & Ständige Impfkommission \\
\hline TSK & Tampa Skale of Kinesiophobia \\
\hline $\mathrm{W}$ & Weiblich \\
\hline
\end{tabular}




\section{Einleitung}

Für die operative Medizin konnte in mehreren Untersuchungen gezeigt werden, dass vorbestehende chronische Schmerzen beziehungsweise schmerzrelevante Komorbiditäten wie Depressivität und Ängstlichkeit Risikofaktoren für eine hohe postoperative Schmerzintensität sind (Ip et al. 2009; Gerbershagen et al. 2014).

Eine chronische Schmerzerkrankung geht für die Patienten mit viel Leid und einer erheblichen Einschränkung der Lebensqualität einher. Dieses spiegelt sich unter anderem in einer eingeschränkten Mobilität und einer schlechten Schlafqualität wider. Außerdem sind die psychische Gesundheit und das soziale Leben beeinträchtigt (Poobalan et al. 2001; Courtney et al. 2002).

Ungenügend behandelte starke Schmerzen können dazu führen, dass der stationäre Aufenthalt der Patienten unnötig verlängert wird (Kehlet 2004; Capdevila et al. 1999). Des Weiteren entstehen weitere erhebliche Kosten für das Gesundheitssystem in der Versorgung von chronischen Schmerzen in der Folge akuter Erkrankungen. Dies wird deutlich am häufigen Auftreten von chronischem postoperativem Schmerz (Frettlöh et al. 2009) oder der Chronifizierung von Rückenschmerzen (Geurts et al. 2018).

Ob vorbestehender chronischer Schmerz, dessen Prävalenz in der Bevölkerung bis zu 28 \% beträgt, auch bei Patienten mit akuten nicht-operativen Schmerzen einen Einfluss auf den klinischen Verlauf hat, ist nicht umfassend untersucht (Häuser et al. 2015; Häuser et al. 2014; Nickel und Raspe 2001; Kurita et al. 2012). Dieses ist wichtig, um gegebenenfalls Optimierungen im Management dieser Patienten daraus abzuleiten, da Verbesserungen in der Versorgung von Schmerzen für Patienten in der konservativen Medizin dringend notwendig sind. Maier et al. 2010 zeigten anhand von Zahlen aus dem Projekt „Schmerzfreies Krankenhaus", dass die Versorgung der Schmerzen in den konservativen Fächern im Vergleich zur Chirurgie besonders problematisch ist.

Ziel dieser prospektiven, monozentrischen Beobachtungsstudie war, den Einfluss vorbestehender chronischer Schmerzen auf den klinischen Verlauf bei Patienten mit akuten Schmerzen darzustellen, die nicht durch eine Operation verursacht wurden, sondern im Kontext einer akuten Erkrankung stehen. Dabei wurden neben der Schmerzintensität auch schmerzbezogene funktionelle Aspekte und psychologische Faktoren berücksichtigt. Als Patientenkollektiv wurden Patienten mit akuter Herpes-Zoster-Infektion ausgewählt, die aufgrund des 
Schweregrades der Infektion stationär behandelt wurden. Die Untersuchung der Fragestellung an Patienten mit akuter Herpes-Zoster-Infektion versprach einen gut abzugrenzenden nicht-operativen Akutschmerz, der in der Regel durch eine hohe Schmerzintensität mit typischer umschreibender Lokalisation gekennzeichnet ist und mit längeren schmerzhaften Verläufen einhergehen kann (Benbernou et al. 2011). Die Verlaufsdaten der Patienten wurden während der stationären Behandlung und im Rahmen einer telefonischen Nachbefragung drei und sechs Monate nach der Entlassung aus der stationären Behandlung erfasst.

Ein weiteres Ziel dieser Studie war es, Risikoindikatoren für starke Akutschmerzen zu identifizierten. Außerdem sollten Risikoindikatoren für ungünstige Langzeitverläufe und die Entwicklung chronischer Zosterschmerzen hinsichtlich ihrer klinischen und funktionellen Bedeutung erfasst werden.

\subsection{Schmerzen}

\subsubsection{Definition}

Die International Association for the Study of Pain (IASP) definiert Schmerz als eine unangenehme sensorische und emotionale Erfahrung, die mit tatsächlichen oder potenziellen Gewebeschädigungen assoziiert ist oder mit Begriffen solcher Schädigungen beschrieben wird (,Pain is an unpleasant sensory and emotional experience associated with actual or potential tissue damage or described in terms of such damage“) (Merskey et al. 1979).

\subsubsection{Nozizeptive und neuropathische Schmerzen}

Schmerzen lassen sich zum einen nach dem Ursprung und zum anderen nach der Dauer einteilen. Bei der Einteilung nach dem Ursprung wird zwischen nozizeptiven und neuropathischen Schmerzen unterschieden (Schaible 2007). Nozizeptive Schmerzen sind somatisch oder viszeral bedingt und entstehen durch eine Verletzung oder Entzündung mit nachfolgender Erregung nozizeptiver Rezeptoren (Pergolizzi et al. 2013).

Neuropathische Schmerzen sind definiert als Schmerzen, die durch eine Läsion oder Erkrankung des somatosensorischen Systems verursacht sind (Colloca et al. 2017). Nach den Kriterien der International Association for the Study of Pain (IASP) (Treede et al. 2008) liegen „sichere" neuropathische Schmerzen vor, wenn die Schmerzen

1. ,eine plausible neuroanatomische Verteilung zeigen (entsprechend dem peripheren / zentralen Innervations-/ Repräsentationsterritorium)“", 
2. „anamnestisch Hinweise auf eine Läsion oder zugrunde liegende Erkrankung bestehen, die das somatosensorische System schädigen kann“ und 3. „,bei denen ein klinischer bzw. apparativer Nachweis von 1. und 2. erfolgt ist.“

Neuropathische Schmerzen entstehen durch eine Irritation oder Schädigung zentraler bzw. peripherer nozizeptiver Systeme durch mechanische, metabolische, toxische oder entzündliche Reaktionen (Baron 2006). Die dadurch resultierenden plastischen Veränderungen im peripheren und zentralen Nervensystem können mit der Zeit irreversibel werden. Die Schmerzen bestehen trotz Gewebeheilung fort (Baron 2000).

Klinisch lassen sich bei neuropathischen Schmerzen negative und positive somatosensorische Symptome unterscheiden. $\mathrm{Zu}$ den negativen somatosensorischen Komponenten zählt man die Verminderung oder den Ausfall einer entsprechenden somatosensorischen Qualität, was sich durch Hypästhesie, Hypalgesie, Thermhypästhesie, Pallhypästhesie, Lagesinnstörung oder Analgesie und Anästhesie äußert. Zu den positiv somatosensorischen Symptomen zählen einerseits Kribbelparästhesien und Dysästhesien und andererseits Allodynie und Hyperalgesie als evozierte Schmerzen (Pergolizzi et al. 2013). Spontan auftretende Schmerzen werden meist als Dauerschmerzen von brennender Qualität und als einschießende, elektrisierende und stechende Schmerzattacken (neuralgiformer Schmerz) beschrieben (Baron 2006).

\subsubsection{Akute und chronische Schmerzen}

Chronische Schmerzen werden einerseits über die Dauer ihres Bestehens definiert. Manche Autoren definieren dabei den chronischen Schmerz als Schmerz, der länger als drei Monate anhält (Wong und Fielding 2011) bzw. länger als sechs Monate anhält (Kurita et al. 2012; Gerbershagen et al. 2009).

Von akuten Schmerzen unterscheiden sich die chronischen Schmerzen zum einen durch die Dauer der Symptome. Doch auch hinsichtlich ihrer Pathophysiologie unterscheiden sich diese beiden Schmerzformen. Charakteristisch für den akuten Schmerz ist, dass dieser meist im Zusammenhang mit dem Auftreten von Gewebeschädigung/-reizung hervorgerufen wird und somit eine Warnfunktion hat. Diesen Zweck der Warnfunktion erfüllt der chronische Schmerz nicht (Zimmermann 2004). 
Chronische, nicht tumorbedingte Schmerzen haben in Deutschland, definiert über die Zeitachse von drei Monaten, eine Prävalenz von 26,9 \%. Für chronische neuropathische Schmerzen konnte eine Prävalenz von 6,9-10 \% der Gesamtbevölkerung gezeigt werden (van Hecke et al. 2014). Bezieht man bei der Erfassung der Prävalenz chronischer Schmerzen starke funktionelle Beeinträchtigungen durch den Schmerz mit ein (entsprechend von Korff Chronic Pain Grade III und IV), beträgt diese 7,4 \% in der Bevölkerung (Häuser et al. 2014). Treten auch noch psychische Symptome hinzu, beträgt die Prävalenz 2,3 \% der Bevölkerung. Die Kombination von Schmerzen, funktioneller Beeinträchtigung und psychischen Faktoren bestimmt das Krankheitsleben der Betroffenen und wird auch als Schmerzkrankheit beschrieben (Häuser et al. 2015).

Neben den funktionellen Beeinträchtigungen leiden Patienten mit chronischen Schmerzen auch unter psychischen Beeinträchtigungen. So konnte beispielsweise für bis zu $21 \%$ der Patienten mit chronischen Schmerzen eine Depression diagnostiziert werden. Mehr als die Hälfte der Patienten hatten durch die chronischen Schmerzen Einschränkungen im Beruf widerfahren (Breivik et al. 2006). Des Weiteren konnte gezeigt werden, dass mehr als die Hälfte der Patienten mit chronischen Schmerzen unter Schlaflosigkeit leiden (Tang et al. 2007). Oft neigen die Patienten auch zur Somatisierung (Birket-Smith 2001).

Am häufigsten werden chronische Schmerzen im Bereich des Rückens angegeben (13,9\%). Weitere häufige Lokalisationen sind die Schulter und Arme (13,2 \%) sowie die Beine (12,2 \%) (Nickel und Raspe 2001; Kurita et al. 2012).

\subsection{Herpes Zoster}

Die jährliche Inzidenz von akuten Herpes-Zoster-Erkrankungen wird mit 4,5/1000 angegeben. Dabei beträgt das mittlere Erkrankungsalter 59,4 Jahre (Yawn und Gilden 2013). Die Inzidenz ist stark altersabhängig und steigt bei Personen zwischen 50 und 54 Jahren von 6,21/1000 auf eine Inzidenz auf 13,2/1000 bei über 90-Jährigen. Frauen sind häufiger betroffen als Männer (11,1/1000 vs. 7,8/1000) (Ultsch et al. 2011).

Einer Zoster Erkrankung voraus geht eine Infektion mit Varizella-Zoster-Viren, die in der Regel in der Kindheit das Krankheitsbild der Windpocken auslöst. Nach der Infektion persistiert das Virus oft jahrzehntelang in den Ganglien der Hirnnerven, den dorsalen Wurzelganglien des gesamten Rückenmarks und den autonomen Ganglien (Yawn und Gilden 2013). 
Das Virus kann sich reaktivieren, wenn die Virus-spezifische zelluläre Immunität mit steigendem Alter herabgesetzt ist oder wenn eine Immunsuppression des Patienten vorliegt (Werner et al. 2017a). Es kommt zu einer Replikation der Varizella-Zoster-Viren in den Ganglien, anschließend gelangen diese entlang der Nerven zur Haut, wo sie dann die auf das Dermatom bezogene Herpes-Zoster-Erkrankung auslösen. Diese ist gekennzeichnet durch Erytheme mit papulo-vesikulären und pustulösen Effloreszenzen (Goßrau 2014).

Da das aktivierte Virus entlang der sensorischen Nerven wandert, entstehen durch die Reinfektion Veränderungen an den betroffenen Nerven. Dies führt zu Nervenzellverlust, Demyelisierung, Sklerose und Wallerschen Degenerationen (Kost und Straus 1996). Hieraus resultieren die neuropathischen Schmerzen (Gilden et al. 2000).

Herpes Zoster tritt häufiger bei immunsupprimierten Patienten auf. Hierzu zählen beispielsweise Patienten, die eine Chemotherapie, Immunsuppressiva oder Cortico-Steroide erhalten und HIV-positive Patienten (Wareham und Breuer 2007). Für das Auftreten einer akuten Herpes-Zoster-Infektion lässt sich keine jahreszeitliche Häufung feststellen (Gilden et al. 2000).

\subsection{Schmerzen bei Herpes-Zoster-Infektionen}

\subsubsection{Akute Schmerzen}

Die Schmerzen, die von einer akuten Herpes-Zoster-Infektion ausgehen, sind in der Regel ein Mischbild aus neuropathischen und nozizeptiven Schmerzen (Allegri et al. 2014; Marchettini et al. 2006; Johnson 2007). Prodromalschmerzen können bereits einige Tage bestehen bevor Hauteffloreszenzen zu sehen sind. Zwischen 68,2 \% und 75\% der Patienten über 50 Jahren mit Herpes Zoster beschreiben Prodromalschmerzen. Im Mittel treten diese Schmerzen fünf Tage vor den ersten Hauteffloreszenzen auf (Song et al. 2014).

Bei über $50 \%$ der Patienten mit Herpes Zoster sind thorakale Dermatome befallen (Benbernou et al. 2011). In absteigender Häufigkeit betroffen sind der Nervus trigeminus (20\%), die cervikalen (11\%), lumbalen (13\%) und sakralen Dermatome (2\%) (Meister et al. 1998b). 
Einfluss auf die Intensität von akuten Zosterschmerzen hat zum einen das Auftreten von Prodromalschmerzen (Benbernou et al. 2011), zum anderen steigt mit dem Alter der Patienten die Schmerzintensität. Auch die Größe des Hautausschlages korreliert positiv mit der akuten Schmerzintensität (Dworkin et al. 2001).

\subsubsection{Post-Zoster-Neuralgie}

Die durch den Herpes Zoster verursachten Schmerzen können chronifizieren und die häufigste Komplikation der Herpes-Zoster-Infektion auslösen: die Post-Zoster-Neuralgie (Gilden et al. 2000). Die Definition der Post-Zoster-Neuralgie ist nicht einheitlich, in Tabelle 1 sind verschiede Definitionen dargestellt.

Tabelle 1 Definitionen der Post-Zoster-Neuralgie

\section{Autor Definition}

Oxmann et al. Schmerzen, die 90 Tage nach der Abheilung des Zosters persistieren 2005 oder wieder auftreten. Als zusätzliches Kriterium kann eine Mindeststärke des Schmerzes von 3 auf einer 0 - 10 NRS verwendet werden

Gilden et al. Schmerzen, die noch sechs Wochen nach dem Erscheinen der Efflores$2000 \quad$ zenzen anhalten

Wareham und Schmerzen, die länger als vier Monate nach der Abheilung der EffloresBreuer 2007 zenzen bestehen

Ein Risikofaktor für das Auftreten einer Post-Zoster-Neuralgie ist unter anderem das Alter der Patienten. 18 \% der Patienten nach dem 50. Lebensjahr und $33 \%$ der Patienten nach dem 80. Lebensjahr entwickeln nach einer Herpes-Zoster-Infektion eine Post-Zoster-Neuralgie (Yawn und Gilden 2013). Weibliches Geschlecht, Prodromalschmerzen, schwerer oder disseminierter Hautausschlag und starker akuter Zosterschmerz sind weitere bekannte Faktoren (Jung et al. 2004; Scott et al. 2003; Whitley et al. 1998; Dworkin et al. 2001). Die Ergebnisse eines umfangreichen Review untermauern die gennannten Risikofaktoren, kommen aber zu dem Ergebnis, dass weibliches Geschlecht kein eindeutiger Risikofaktor zu sein scheint. Das Review erweitert die Risikofaktoren aber um das Vorliegen eines Zoster ophthalmicus (Forbes et al. 2016a). Ferner wird die Lokalisation des Herpes Zoster im kranialen oder sakralen Bereich als ein Risikofaktor betrachtet (Meister et al. 1998a). 


\subsection{Therapieoptionen des akuten Herpes Zoster}

\subsubsection{Antivirale Therapie}

Entsprechend der aktuellen S2k Leitlinie „Guideline on the Management of Herpes Zoster” des European Dermatology Forum (EDF) (Werner et al. 2017b) sollen alle an Herpes Zoster erkrankten Patienten ab dem 50. Lebensjahr antiviral behandelt werden. Eine antivirale Therapie ist ebenfalls indiziert bei Herpes Zoster im Kopf-Halsbereich, bei moderaten bis starken Schmerzen, hämorrhagischen oder nekrotisierenden Läsionen, bei Befall von mehr als einem Dermatom und Schleimhautbeteiligung. Des Weiteren soll entsprechend der Leitlinie Herpes Zoster bei immungeschwächten Patienten, bei Patienten mit schwerer Dermatitis atopica und bei Kindern, die Salizylate oder Kortikosteroide als Dauertherapie erhalten, antiviral therapiert werden. Eine relative Indikation zur antiviralen Therapie liegt bei Patienten jünger als 50 Jahre mit Herpes Zoster am Stamm oder an den Extremitäten vor. Als antivirale Mittel eignen sich die hierfür zugelassenen Medikamente Aciclovir, Brivudin, Famciclovir und Valaciclovir.

\subsubsection{Therapie mit Analgetika}

Die aktuelle S2k Leitlinie „Guideline on the Management of Herpes Zoster” (Werner et al. 2017b) empfiehlt eine Schmerztherapie, die sich an dem WHO Stufenschema orientiert. Dabei werden in der ersten Stufe nichtsteroidale Analgetika bzw. Nicht-Opioide verabreicht. In der zweiten Stufe werden zusätzlich schwach wirksame Opioide eingesetzt. Stufe drei beinhaltet die Applikation von stark wirksamen zentralen Opioiden in der Kombination mit einem Nicht-Opioid.

\subsubsection{Therapie mit Co-Analgetika}

Aufgrund der neuropathischen Schmerzkomponente empfiehlt die aktuelle S2k Leitlinie „Guideline on the Management of Herpes Zoster" (Werner et al. 2017b) eine Therapie mit Trizyklischen Antidepressiva (z. B. Amitriptylin) oder Antiepileptika (z. B. Gabapentin, Pregabalin) zusätzlich zu den in Abschnitt 1.4.2 genannten Analgetika. Antidepressiva und Antiepileptika sind die first-line Therapie neuropathischer Schmerzen (Colloca et al. 2017). 


\subsection{Prävention und Therapie der Post-Zoster-Neuralgie}

\subsubsection{Prävention der Post-Zoster-Neuralgie}

Die Kombination aus antiviraler Therapie und Gabapentin scheint das Risiko, eine PostZoster-Neuralgie zu entwickeln, zu reduzieren (Lapolla 2011). Ob und welchen Effekt eine analgetische Therapie des akuten Herpes Zoster in Hinblick auf die Prävention der PostZoster-Neuralgie hat, ist bisher ungeklärt (Goßrau 2014). Die letztendlich einzige gut beschriebene Prävention der Post-Zoster-Neuralgie ist die Vorbeugung der Herpes-Zoster-Infektion durch Zostervakzine (Solomon et al. 2014). In der Mitteilung „Wissenschaftliche Begründung zur Empfehlung einer Impfung mit dem Herpes-zoster-subunit-Totimpfstoff“ der Ständigen Impfkommission (STIKO) beim Robert Koch Institut (Siedler et al. 2019) wird eine Impfung mit adjuvantierten Herpes-zoster-subunit-(HZ/su-) Totimpfstoff für die Prävention von Herpes Zoster und der Post-Zoster-Neuralgie für alle Personen älter als 60 Jahre empfohlen. Des Weiteren empfiehlt die STIKO eine Impfung von Personen älter als 50 Jahre, die eine erhöhte gesundheitliche Gefährdung infolge einer Grundkrankheit haben (z. B. angeborene oder erworbene Immundefizienz oder Immunsuppression, HIV-Infektion, Rheumatoider Arthritis, Systemischem Lupus erythematodes, Chronisch entzündliche Darmerkrankungen, Chronisch obstruktive Lungenerkrankungen oder Asthma bronchiale, Chronische Niereninsuffizienz, Diabetes mellitus).

\subsubsection{Therapieoptionen der Post-Zoster-Neuralgie}

Die Post-Zoster-Neuralgie zählt zu den chronischen neuropathischen Schmerzen (Goßrau 2014). Daher ist, analog zu anderen chronischen Schmerzen, oft ein multimodaler Therapieansatz erforderlich. Zur Therapie der Post-Zoster-Neuralgie eignen sich systemische und topische Medikamente. Wie bei anderen Schmerzerkrankungen hängt die Wahl des eingesetzten Medikaments vom individuellen Schmerzbild, den vorhandenen Kontraindikationen und den Nebenwirkungen ab (Goßrau 2014).

Tabelle 2 gibt einen Überblick über die Medikamente, die sich in randomisiert kontrollierten Studien als wirksam in der Therapie der Post-Zoster-Neuralgie gezeigt haben (Edelsberg et al. 2011). 
Tabelle 2 Medikamentöse Therapieoptionen der Post-Zoster-Neuralgie

\begin{tabular}{l|l} 
Klasse & Wirkstoff \\
\hline \hline trizyklische Antidepressiva & Amitriptylin, Nortriptylin, Desipramin \\
Antikonvulsiva & Gabapentin, Pregabalin \\
Opioidanalgetika & Tramadol, Morphin retardiert, Oxycodon
\end{tabular}

Die Wirksamkeit der topisch anwendbaren Medikamente Lidocain (als Pflaster) und Capsaicin (als Pflaster und Salbe) wurde in klinischen Studien nachgewiesen (Goßrau 2014). Eine weitere Therapieoption in der Behandlung der Post-Zoster-Neuralgie besteht in der Anwendung von transkutaner elektrischer Nervenstimulation, welche als lokales Verfahren ohne systemische Nebenwirkungen gut eingesetzt werden kann. Auch die Psychotherapie wird im Rahmen eines multimodalen Therapiekonzeptes als sinnvoll erachtet (Goßrau 2014).

\subsection{Prädiktoren für akute und chronische postoperative Schmerzen}

Für den operativen Bereich ist sehr umfangreich untersucht worden, welche Prädiktoren es für akute und chronische postoperative Schmerzen gibt. Der folgende Abschnitt gibt hierüber einen Überblick. Dieses ist wichtig, da in dieser Studie auch Risikoindikatoren überprüft wurden, die als etablierte Prädiktoren für akute beziehungsweise chronische Schmerzen aus dem operativen Bereich bekannt sind.

\subsubsection{Akuter postoperativer Schmerz}

Vorbestehende, das heißt präoperativ bereits bestehende Schmerzen sind ein etablierter Prädiktor für starke postoperative Schmerzen (Gerbershagen et al. 2009; Ip et al. 2009; Melotti et al. 2005). Weitere Prädiktoren für starke postoperative Akutschmerzen sind in Tabelle 3 aufgeführt. 
Tabelle 3 Prädiktoren für starke postoperative Akutschmerzen

\begin{tabular}{l||ll} 
Autor & Gerbershagen et al. 2014 & Ip et al. 2009 \\
\hline Prädiktor & - Präoperativer chronischer Schmerz & - Präoperative Schmerzen \\
& - weibliches Geschlecht & - geringeres Alter \\
& - geringeres Alter & - Angst \\
& & - Operationsverfahren
\end{tabular}

Starke postoperative Schmerzen gehen einher mit einem schlechteren postoperativen Outcome, möglicherweise der Entwicklung von starken chronischen Schmerzen, einer verzögerten Rehabilitation und einer Verminderung der Lebensqualität (Peters et al. 2007).

\subsubsection{Chronischer postoperativer Schmerz}

Die Entwicklung von chronischen Schmerzen nach Operationen ist zum einen abhängig von der Art und Technik des operativen Eingriffs (Schnabel und Pogatzki-Zahn 2010). Auf Operationen, bei denen es zu einer iatrogenen Schädigung von Nerven kommt, liegt ein besonderer Augenmerk, denn es ist davon auszugehen, dass ein Großteil der Patienten mit chronischen Schmerzen nach Operationen eine neuropathische Schmerzkomponente entwickelt (Mikkelsen et al. 2004; Jung et al. 2003). So konnten beispielsweise bei $66 \%$ der Patienten, die nach einer Thorax-Operation persistierende postoperative Schmerzen hatten, eine neuropathische Schmerzkomponente nachgewiesen werden (Haroutiunian et al. 2013). Weitere Prädiktoren sind im Wesentlichen die gleichen wie die Prädiktoren für einen starken postoperativen Schmerz. Hierzu zählen vorbestehende Schmerzen, junges Alter, weibliches Geschlecht und psychosoziale Faktoren (Schnabel und Pogatzki-Zahn 2010). Ein weiterer etablierter Prädiktor für das Auftreten von chronischen postoperativen Schmerzen ist das Vorhandensein von starken akuten Schmerzen nach der Operation (Schnabel und Pogatzki-Zahn 2010; Althaus et al. 2012).

$\mathrm{Zu}$ den psychosozialen Faktoren, die mit dem Vorhandensein von chronischen Schmerzen einher gehen, zählen das Vorhandensein von Depression und Stress, eine psychologische Vulnerabilität sowie ein spätes Zurückkehren an den Arbeitsplatz (Hinrichs-Rocker et al. 2009). Außerdem hat sich Katastrophisieren als ein robuster Faktor in der Prädiktion von 
chronischen Schmerzen nach Operationen erwiesen (Katz und Seltzer 2009). Auch genetische Prädispositionen scheinen eine wichtige Rolle zu spielen. Als ein Beispiel hierfür sei das unterschiedliche Ansprechen von Patienten auf Analgetika erwähnt (Kehlet et al. 2006). Zusammenfassend lässt sich festhalten, dass bei der Chronifizierung von Schmerzen vielschichtige somatische, psychische und soziale Mechanismen zusammenwirken (Zimmermann 2004; Hasenbring et al. 2001). 


\section{Patienten und Methoden}

\subsection{Patienten}

Für diese prospektive, monozentrisch beobachtende Kohortenstudie wurden 78 Patienten im Zeitraum von April 2012 bis Juni 2014 zur Teilnahme aufgefordert.

In die Studie wurden alle weiblichen und männlichen Patienten eingeschlossen, die in der Dermatologie der Universitätsmedizin Göttingen aufgrund einer akuten Herpes-Zoster-Erkrankung stationär aufgenommen wurden. Die Patienten mussten mindestens 18 Jahre alt sein sowie geistig und rechtlich in der Lage sein, zur Teilnahme an der Studie einzuwilligen. Außerdem mussten die Patienten ausreichend Deutschkenntnisse besitzen, um die Patienteninformation und die Fragebögen zu verstehen bzw. beantworten zu können.

Die Diagnose der akuten Herpes-Zoster-Infektion wurde anhand klinischer Beurteilung der Patienten durch die Ärzte der Klinik für Dermatologie gestellt. Die Einbeziehung von Laborparametern zur Verifikation der Diagnose erfolgte nicht.

Ausgeschlossen wurden alle Patienten, die die oben genannten Kriterien nicht erfüllten. Ebenfalls nicht in die Studie eingeschlossen wurden Patienten, die zum Zeitpunkt der Aufnahme eine vorbekannte Tumorerkrankung aufwiesen, eine immunsupprimierende Grunderkrankung (einschließlich HIV-positive Patienten) hatten und Patienten mit aktiven Substanzabusus.

Das Studienprotokoll wurde der Ethikkommission der medizinischen Fakultät der Universität Göttingen (29/5/12) vorgelegt und beraten. Die Studie erfolgte nach den aktuellen Richtlinien der Deklaration von Helsinki.

\subsection{Ablauf der Studie}

Nach der Aufnahme der Patienten auf die dermatologischen Stationen der Universitätsmedizin Göttingen erfolgte möglichst zeitnah, je nach Aufnahmezeitpunkt jedoch spätestens innerhalb von 24 Stunden, der Einschluss und die Aufnahme der Patienten in die Studie. In Abbildung 1 ist der Flow Chart des Studienablaufs dargestellt. 
Patientenrekrutierung, Information, Aufklärung, Einwilligung gescreent $n=78$, Einschluss $n=63$

$\sqrt{2}$

Schmerzanamnese, Erfassung von schmerzrelevanten Risikofaktoren, Funktionen, Analgetikabedarf n=59 (n=3 Rückzug Teilnahme, $n=1$ andere Schmerzdiagnose)

$\sqrt{2}$

Messung der individuellen Schmerzschwelle

(Druckschmerzschwelle)

ת

Verlaufsparameter an Tag 4 und 7

$\sqrt{3}$

Abschlussuntersuchung sowie Reevaluation schmerzrelevanter Risikofaktoren bei Entlassung

\

1. Nachbefragung 3 Monate nach der stationären Behandlung zu Schmerz, Funktion und Lebensqualität, Analgetikabedarf $n=50$ ( $n=8$ lost to $F U)$

\

2. Nachbefragung 6 Monate nach der stationären Behandlung zu Schmerz, Funktion und Lebensqualität, Analgetikabedarf $n=49$ (n=1 lost to $F U)$

$\sqrt{2}$

\section{Abschließende Datenanalyse}

Abbildung 1 Flow Chart des Studienablaufs 


\subsection{Methoden}

\subsubsection{Schmerzanamnese}

Anhand einer modifizierten Form des Deutschen Schmerzfragebogens erfolgte eine standardisierte Schmerzanamnese (Nagel et al. 2002). Dabei wurde unterschieden zwischen akuten Schmerzen, die von der Herpes-Zoster-Infektion ausgehen, und chronischen Schmerzen, die unabhängig vom Herpes Zoster vorbestanden. Die von der Herpes-Zoster-Infektion ausgehenden Schmerzen wurden definiert als Schmerzen, die im Zuge der Infektion neu aufgetreten waren und sich auf das vom Herpes Zoster infizierte Hautareal bzw. Dermatom bezogen. Als vorbestehende, vom Zoster unabhängige chronische Schmerzen wurden alle Schmerzen definiert, die mindestens über sechs Monate vor der akuten Herpes-Zoster-Infektion bestanden.

$\mathrm{Zu}$ jeder der angegebenen Schmerzlokalisation wurden analog zum Deutschen Schmerzfragebogen (DSF) der Schmerzcharakter (z. B. brennend, stechend, furchtbar) und die Dauer der Schmerzen (weniger als 1 Monat; 1 Monat bis $1 / 2$ Jahr; $1 / 2$ Jahr bis $1 \mathrm{Jahr} ; 1$ bis 2 Jahre; 2 bis 5 Jahre; mehr als 5 Jahre) erfasst und soweit möglich eine klinische Diagnose der Schmerzursache angegeben. Außerdem wurden erfasst die Häufigkeit der Schmerzen, der Schmerzverlauf, bisherige Therapieversuche, die Sozialanamnese und die Schmerzmedikation.

\subsubsection{Graduierung des Schweregrades und des Chronifizierungsstadiums der chronischen Schmerzerkrankung}

Mit Hilfe der deutschen Version des Chronic Pain Grade Questionnaire nach von Korff wurde bei vorliegenden chronischen Schmerzen der Schweregrad der chronischen Schmerzerkrankung des Patienten in vier Kategorien eingeteilt (von Korff et al. 1992). Grundlage dieser Einteilung ist die Berücksichtigung der Schmerzintensität, die Beeinträchtigung in den täglichen Aktivitäten des Patienten durch die Schmerzen und die Anzahl der Tage, an denen der Patient aufgrund der vorbestehenden Schmerzen in seiner Arbeitsfähigkeit (inklusive Hausarbeit) beeinträchtigt war.

Grad 1 entspricht einer geringen Beeinträchtigung mit einer geringen Schmerzintensität. Grad 2 entspricht einer geringen Beeinträchtigung mit einer hohen Schmerzintensität. Diese beiden Grade entsprechen klinisch einem funktionalen chronischen Schmerz. In Grad 3 liegt eine mäßige, in Grad 4 eine starke Beeinträchtigung des Patienten durch die Schmerzen vor. 
Die Einordnung in diese Grade erfolgte unabhängig von der Schmerzintensität, klinisch entsprechen die Grade 3 und 4 einem dysfunktionalen chronischen Schmerz. Grad 0 wurden diejenigen Patienten zugeteilt, die weder Schmerzen noch eine Beeinträchtigung angegeben haben.

Durch die in der Schmerzanamnese ermittelten Informationen wurden die chronischen Schmerzen der Patienten anhand des Chronifizierungsgrades nach Gerbershagen (Mainz Pain Staging System, MPSS) in drei Stadien eingeteilt. Die Stadien errechnen sich aus der Beurteilung von insgesamt vier Achsen: Achse 1 charakterisiert zeitliche Aspekte des Schmerzes (Auftretenshäufigkeit, Dauer und Intensitätswechsel des Schmerzes), Achse 2 die räumlichen Aspekte (monolokulär, bilokulär oder multilokulär auftretende Schmerzen), Achse 3 das Medikamenteneinnahmeverhalten (Analgetikabedarf und Anzahl der Entzugsbehandlungen) und Achse 4 die Patientenkarriere (Anzahl der Arztwechsel, schmerzbezogene Krankenhausaufenthalte, schmerzbedingte Operationen) (Schmitt und Gerbershagen 1990).

\subsubsection{Neuropathische Schmerzcharakteristika}

Der Fragebogen painDETECT wurde zunächst im Rahmen eines epidemiologischen Erfassungsprojektes an Patienten mit postherpetischer Neuralgie und diabetischer Polyneuropathie entwickelt und gilt inzwischen als etabliertes Screening-Instrument zur Erfassung neuropathischer Schmerzkomponenten. Für diese Studie wurde eine modifizierte Version mit einem Bezugsrahmen von drei Monaten statt vier Wochen verwendet. Der Fragebogen bezieht sich auf die durch Herpes Zoster verursachten Schmerzen und liefert differenzierte Angaben zur durchschnittlichen und maximalen Intensität der Zosterschmerzen innerhalb der letzten drei Monate sowie der aktuellen Schmerzintensität. Außerdem werden Lokalisation, Art und Verlauf der Schmerzen sowie etwaige Ausstrahlung der Schmerzen erfasst. Ferner werden sieben Fragen zur Schmerzqualität gestellt, die mit einer Skala von „nie“ bis „sehr“ beantwortet werden. Für jede Frage des Tests werden je nach Ausprägung des Parameters Punkte vergeben und addiert. Die maximal erreichbare Punktsumme ist 38. Durch die Auswertung des Fragebogens werden die Schmerzen des Patienten in drei Stufen eingeteilt: Ab 19 Punkten ist ein Vorliegen einer neuropathischen Schmerzkomponente wahrscheinlich. Werte zwischen 13 und 18 Punkten lassen keine Aussage zu, ob eine neuropathische Schmerzkomponente vorliegt. Bei zwölf oder weniger Punkten ist eine neuropathische Schmerzkomponente unwahrscheinlich (Freynhagen et al. 2006; Bennett et al. 2007). 


\subsubsection{Aktuelle Schmerzintensität und schmerzbedingte Einschränkungen}

Das validierte Fragemodul QUIPS (Qualitätsverbesserung in der postoperativen Schmerztherapie) entstand im Rahmen eines durch das Bundesministerium für Gesundheit geförderten Projektes zur Qualitätssteigerung im Gesundheitswesen. Es umfasst Fragen zur Schmerzintensität (stärkste und geringste Schmerzen am Tag der Erhebung, Schmerzen bei Belastung), die mittels einer Numerischen Rating Skala (NRS) von 0 (,,kein Schmerz“) bis 10 (,stärkster vorstellbarer Schmerz") beantwortet werden sowie Fragen zur Zufriedenheit (NRS 0-15) der Patienten mit der Schmerzbehandlung. Neben dem Verlangen nach weiteren Schmerzmitteln (ja/nein) wird die Beeinträchtigungen des Patienten durch die Schmerzen (Beeinträchtigung in der Mobilität und der Stimmung, Schmerzen beim Husten, Aufwachen durch Schmerzen) und durch Schmerzmittelnebenwirkungen (Vorhandensein von Müdigkeit, Übelkeit und Erbrechen) erfragt.

Im Rahmen der postoperativen Schmerztherapie ist der QUIPS inzwischen als Standardfragebogen etabliert (Meissner et al. 2008). Der Fragebogen ist validiert für den ersten postoperativen Tag. Er wurde in einer für die dermatologischen Patienten modifizierten Version genutzt, sodass die akuten Zosterschmerzen für die jeweiligen Tage erfasst werden konnten.

\subsubsection{Quantifizierung der Schmerzmedikation}

Die Erfassung der Schmerzmedikation erfolgte zum einen für den Aufnahmetag, Tag 4 und 7 der stationären Behandlung sowie den Entlassungstag und bezieht sich auf alle Schmerzmedikamente, die die Patienten in der Klinik erhielten. Sie wurden aus der Patientenkurve übernommen. Zum anderen erfolgte die Erfassung der analgetischen Vormedikation. Hierunter fallen alle Schmerzmedikamente, die die Patienten bereits vor der Herpes-Zoster-Infektion erhielten. Die Schmerzmedikation wurde durch den Medication Quantification Score (MQS) quantifiziert. Diese Score ermöglicht die statistische Vergleichbarkeit des Schmerzmittelkonsums von Patienten. Der MQS errechnet sich aus dem für jedes Analgetikum spezifischen Schädigungsgrad (SG) multipliziert mit dem Dosislevel, welches mit zunehmender Dosis steigt. Die Analgetika wurden unterteilt in Opioide, Nicht-Opioide und Co-Analgetika (Antidepressiva und Antikonvulsiva). Für diese Gruppen wurde der MQS-Wert berechnet, in dem alle der Gruppe zugehörigen MQS-Werte der Analgetika addiert wurden. Den Gesamt-MQS der von den Patienten konsumierten Schmerzmittel erhält man durch die Summierung der Gruppen MQS-Werte (Stormo et al. 1998). Für die Errechnung des MQS wurden die in Tabelle 4 dargestellten Werte verwendet. 
Für die Opioide wurde zusätzlich zur besseren Vergleichbarkeit das orale Morphinäquivalent errechnet. Hierfür wurden folgende Umrechnungsfaktoren benutzt: Umrechnungsfaktoren für Morphin per os: Tramadol 10, Tilidin 10, Dihydrocodein 4, Oxycodon 0,75, Hydromorphon 0,13, Buprenorphin 0,03, Fentanyl TTS ( $\mu \mathrm{g} / \mathrm{h})$ 0,01. 
Tabelle 4 MQS Umrechnungstabelle

\begin{tabular}{|c|c|c|c|c|c|}
\hline Level & S G & 1 & 2 & 3 & 4 \\
\hline \multicolumn{6}{|l|}{ Opioide } \\
\hline $\begin{array}{l}\text { Tramal / Tilidin (mg, } \\
\text { MÄ) }\end{array}$ & 2,3 & $\begin{array}{l}<=100 \\
(10)\end{array}$ & $\begin{array}{c}101-299(10,1- \\
29,9)\end{array}$ & $\begin{array}{l}300-600(30- \\
60)\end{array}$ & $\begin{array}{l}>600 \\
(>60)\end{array}$ \\
\hline starke Opioide (mg, MÄ) & 3,4 & $<=10$ & $10,1-59,9$ & $60-120$ & $>120$ \\
\hline \multicolumn{6}{|l|}{ Nicht-Opioide } \\
\hline Paracetamol (mg) & 2,2 & $<=500$ & $<=1500$ & $1501-3000$ & $>3000$ \\
\hline Metamizol (mg) & 2,3 & $<=500$ & $<=1500$ & $<=5000$ & $>5000$ \\
\hline Diclofenac (mg) & 3,4 & 0 & $<=50$ & $75-100$ & $>150$ \\
\hline Ibuprofen (mg) & 3,4 & $600-800$ & 1000 & 1200 & 2400 \\
\hline ASS (mg) & 3,4 & 500 & $501-2000$ & $2001-4000$ & $>4000$ \\
\hline Entoricoxib (mg) & 2,3 & NA & 60 & 90 & 120 \\
\hline Parecoxib (mg) & 2,3 & NA & 40 & 80 & $>80$ \\
\hline \multicolumn{6}{|l|}{ Co-Analgetika } \\
\hline Pregabalin (mg) & 1,9 & $<=75$ & $<=300$ & $<=600$ & $>600$ \\
\hline Gabapentin (mg) & 1,9 & $<=300$ & $<=1200$ & $1200-3600$ & $>3600$ \\
\hline Amitryptilin (mg) & 2,3 & $<=10$ & $10-50$ & $50-75$ & $>75$ \\
\hline Citalopram (mg) & 1,7 & 0 & $<=20$ & $20-40$ & $>40$ \\
\hline Lorazepam (mg) & 3,9 & 0,5 & 1 & 2,5 & $>2,5$ \\
\hline Trimipramin (mg) & 2,3 & 5 & $10-50$ & $50-75$ & $>75$ \\
\hline Mirtazapin (mg) & 1,9 & $<=7,5$ & 15 & 30 & $>30$ \\
\hline Duloxetin (mg) & 1,9 & NA & $<60$ & $60-120$ & $>120$ \\
\hline Sertralin (mg) & 1,7 & NA & $<100$ & $100-200$ & $>200$ \\
\hline Fluoxetin (mg) & 1,7 & NA & $<=30$ & $31-60$ & $>60$ \\
\hline Venlafaxin (mg) & 1,9 & NA & 37,5 & $75-150$ & $>150$ \\
\hline Tetrazepam (mg) & 3,9 & 50 & $51-100$ & $101-150$ & $>150$ \\
\hline
\end{tabular}




\subsubsection{Gesundheitsbezogene Lebensqualität}

Der SF 12 ist die Kurzform des Health Survey Questionnaire SF 36 und setzt sich aus zwölf Fragen zur Selbsteinschätzung der subjektiven Gesundheit zusammen. In Studien wurde eine sehr gute Reliabilität und Validität bescheinigt, sodass der Health Survey Questionnaire als Standard zur Erfassung der subjektiv erlebten Lebensqualität gilt (Jenkinson et al. 1997). Die Auswertung liefert Summenwerte für eine körperliche und eine psychische Summenskala.

\subsubsection{Depression, Angst und Stress}

Die Depression Anxiety Stress Scale (DASS) wurde 1995 entwickelt und ermöglicht anhand von 21 Fragen die Messung und Unterscheidung von den Merkmalen Angst, Depression und Stressbelastung (je 7 Items) (Lovibond und Lovibond 1995; Brown et al. 1997). Das Verfahren ist auch in einer deutschsprachigen Version validiert. Aufgrund der aktuellen Datenlage werden für den DASS folgende Grenzwerte vorgeschlagen: Der problematische Wert (Cut-off) für eine Depression (erhöhte Wahrscheinlichkeit für das Vorliegen einer depressiven Störung) beträgt 10 Punkte. Der Cut-off für eine Angst-Störung liegt bei 6 Punkten und bei 10 Punkten für Stress (Nilges und Essau 2015).

\subsubsection{Bewegungsbezogene Angst}

Die deutsche Version der Tampa Scale of Kinesiophobia (TSK) erfasst Angst vor Bewegungen. Diese Bewegungsangst ist bekannt als ein wichtiger Chronifizierungsfaktor bei muskulo-skelettalen Schmerzen. Es können 17 Aussagen zum Zusammenhang zwischen Bewegung und Angst in vier Antwortstufen (von „trifft überhaupt nicht zu“ bis ,trifft völlig zu“) bewertet werden. Jede Antwortmöglichkeit ist einem bestimmten Zahlenwert zugeordnet. Diese werden zur Auswertung addiert und bilden den Summenscore, der Werte von 17 bis 68 umfasst (Houben et al. 2005). Hohe Summenscores korrelieren positiv mit dem Vorhandensein einer Kinesiophobie (Roelofs et al. 2004). Kinesiophobie ist definiert als "an irrational and debilitating fear of physical movement and activity resulting from a feeling of vulnerability to painful injury or re-injury" (zu Deutsch: irrational und belastende Angst vor Bewegung und körperlicher Aktivität aufgrund von einem Gefühl der Verwundbarkeit in Hinblick auf schmerzvolle Verletzungen oder erneute Verletzungen) (Hapidou et al. 2012). 


\subsubsection{Somatisierung}

Der Gesundheitsfragebogen für Patienten (PHQ-D) ist die deutsche Version des Patient Health Questionnaire (PHQ). Der PHQ-D wird als Screening Instrument für insgesamt acht psychische Störungen verwendet und ist im Sinne eines Modulsystems aufgebaut. Die Module können für die einzelnen Störungsbereiche flexibel entsprechend der jeweiligen Fragestellung zusammengestellt werden. In der Studie wurde die 15 Items umfassende Somatisierungsskala genutzt, um die Somatisierungstendenz der untersuchten Patienten zu erfassen. Pro Item werden entweder 0 (,nicht beeinträchtigt" $\left.{ }^{\circ}\right), 1$ (,wenig beeinträchtigt" ${ }^{6)}$ oder 2 Punkte (,stark beeinträchtigt“) vergeben. Die Punkte werden addiert, sodass die maximale Punktzahl 30 ist. Werte $<5$ entsprechen keiner Somatisierungstendenz, Werte $\geq 5$ entsprechen einer geringen, Werte $\geq 10$ einer mittleren und Werte $\geq 15$ entsprechen einer starken Somatisierungstendenz (Kroenke et al. 2002).

\subsubsection{Katastrophisieren und aktive Bewältigung}

Zur Erfassung der Faktoren schmerzbezogene „aktive Bewältigung“ und Katastrophisieren wurden diese beiden Subskalen aus dem Kieler Schmerzinventar (Kieler Pain Inventory, KPI) als unabhängige Faktoren entnommen. Die individuelle Schmerzverarbeitung auf kognitiver Ebene wird mit insgesamt 18 Items erfasst und gliedert sich in die Kategorien Hilfsund Hoffnungslosigkeit (9 Items), Katastrophisieren (5 Items) und Durchhalteappell (4 Items) (Hasenbring 1994).

Jede Aussage spiegelt Gedanken und Erfahrungen zum Thema Schmerzen in den letzten 14 Tagen wider und kann mit 0 (,nie“) bis 6 (,jedes Mal“) bewertet werden. Diese Werte werden addiert. Hohe Gesamtwerte sprechen für ein häufiges Auftreten von oben genannten Schmerzverarbeitungsmustern.

\subsubsection{Bestimmung der Schmerzschwelle}

Für die Schmerzschwellenmessung wurde mit einem Druckalgometer (Fläche $1 \mathrm{~cm}^{2}$, Hersteller: Somedic Algometer, Stockholm, Schweden, CE-Zertifizierung vorhanden, Einsatz in der Studie gemäß der vorgesehenen und zertifizierten Anwendung) an folgenden Stellen Druck appliziert: lateraler Epicondylus, M. trapezius, M. quadriceps femoris, M. tibialis anterior und auf den Daumennagel. Von Zoster befallende Areale wurden ausgenommen. Die Messungen erfolgten auf beiden Körperseiten. Dabei wurde der Druck senkrecht zu Haut 
ausgeübt und so lange linear mit $50 \mathrm{kPa} / \mathrm{Sek}$. gesteigert, bis der Patient den Reiz als schmerzhaft beschrieb. Der maximal applizierte Druck betrug $1000 \mathrm{kPa}$. Aus den gemessenen Werten wurde der Mittelwert als individuelle Schmerzschwelle errechnet. 


\subsubsection{2 Übersicht der erhobenen Parameter während der Studie}

Tabelle 5 Übersicht der erhobenen Parameter während der Studie

\begin{tabular}{|c|c|}
\hline Zei & arameter \\
\hline $\begin{array}{l}\text { Aufnahme- } \\
\text { tag }\end{array}$ & $\begin{array}{l}\text { - } \text { Schmerzanamnese (Deutscher Schmerzfragebogen) } \\
\text { - } \quad \text { Graduierung der chronischen Schmerzerkrankung (Chronic } \\
\text { - } \quad \text { Neuin Grade) und des Chronifizierungsstadiums (MPSS) } \\
\text { - } \quad \text { Schmerzintensität und Funktionalität (modifizierter QUIPS) } \\
\text { - } \quad \text { Gesundheitsbezogene Lebensqualität (SF 12) } \\
\text { - } \quad \text { Depression, Angst und Stress (DASS) } \\
\text { - Somatisierung (PHQ) } \\
\text { - Bewegungsbezogene Angst (Tampa Scale for Kinesiophobia) } \\
\text { - Katastrophisieren und aktive Bewältigung (KPI) } \\
\text { - Druckschmerzschwelle } \\
\text { - Schmerzmedikamente (MQS) } \\
\text { Therapie und Komplikationen }\end{array}$ \\
\hline $\begin{array}{l}\text { Tag } 4 \text { und } \\
7\end{array}$ & $\begin{array}{ll}\text { - } & \text { Schmerzintensität und Funktionalität (modifizierter QUIPS) } \\
\text { - } & \text { Schmerzmedikamente (MQS) } \\
\text { - } & \text { Therapie und Komplikationen }\end{array}$ \\
\hline $\begin{array}{l}\text { Entlas- } \\
\text { sungstag }\end{array}$ & $\begin{array}{ll}\text { - } & \text { Schmerzintensität und Funktionalität (modifizierter QUIPS) } \\
\text { - } & \text { Neuropathische Schmerzcharakteristika (painDETECT) } \\
\text { - } & \text { Depression, Angst und Stress (DASS) } \\
\text { - } & \text { Bewegungsbezogene Angst (Tampa Scale for Kinesiophobia) } \\
\text { - } & \text { Somatisierung (PHQ) } \\
\text { - } & \text { Schmerzmedikamente (MQS) } \\
\text { - } & \text { Therapie und Komplikationen }\end{array}$ \\
\hline $\begin{array}{l}\text { Nach } 3 \text { und } \\
6 \text { Monaten }\end{array}$ & $\begin{array}{l}\text { - } \text { Schmerzanamnese (Deutscher Schmerzfragebogen) } \\
\text { - } \quad \text { Neuropathische Schmerzcharakteristika (painDETECT) } \\
\text { - Graduierung der chronischen Schmerzerkrankung (Chronic } \\
\text { Pain Grade) } \\
\text { - } \text { - Sesundheitsbezogene Lebensqualität (SF 12) } \\
\text { - Therapie und Komplikationen }\end{array}$ \\
\hline
\end{tabular}




\subsection{Auswertung}

Die statistische Auswertung der vorliegenden Arbeit erfolgte nach Beratung durch das Institut für Medizinische Statistik der Universitätsmedizin Göttingen.

Prozente sind auf ganze Zahlen gerundet dargestellt. Der Mittelwert wurde für kontinuierliche Variablen benutzt, der Median für kategorisierte Variablen. Für den Mittelwert ist die Standardabweichung und für den Median die Quartile als Streumaß angegeben. Häufigkeiten im Gruppenvergleich wurden mit dem Chi-Quadrat-Test nach Pearson verglichen. Der Vergleich zwischen zwei Gruppen wurde bei kategorisierten Variablen mit dem Mann-Whitney-U-Test durchgeführt. Der t-Test wurde für den Vergleich zweier unabhängigen Stichproben bei kontinuierlichen Werten verwendet. Die Varianzhomogenität wurde durch den Levene-Test geprüft und der t-Test entsprechend angepasst. Das Signifikanz Level wurde auf $\mathrm{p}=<0,05$ festgesetzt. Aufgrund des explorativen Charakters der Studie wurden keine weiteren Korrekturen für multiple Vergleiche vorgenommen.

Univariate Analysen wurden mit dem Kendall Tau Test berechnet. Für multivariate Analysen wurde die schrittweise multiple Regression (Rückwärtselemination) verwendet.

Für n=7 Patienten, die vor dem 7. Tag entlassen wurden, entfällt die Berechnung der Verlaufsparameter (modifizierter QUIPS, Schmerzmedikamente MQS) für Tag 7 und es wurde der Wert des Entlassungstags genutzt. Dieses ist nötig, um die Verlaufsparameter bestmöglich chronologisch darzustellen.

\subsubsection{Berechnung der Einflussfaktoren auf die Intensität des akuten Zoster- schmerzes, bezogen auf das gesamte Patientenkollektiv}

Um Variablen ausfindig zu machen, die mit der maximalen Schmerzintensität aller Patienten assoziiert sind, erfolgte zunächst eine univariate Analyse für die jeweils betrachteten Tage: Aufnahmetag, Tag 4 und Tag7 / Entlassung. Dabei wurden die in Tabelle 13 aufgeführten Parameter berücksichtigt. Signifikante Korrelationen aus der univariaten Analyse wurden in eine multivariate Analyse einbezogen (vgl. Kapitel 3.3.1). 


\subsubsection{Berechnung der Einflussfaktoren auf die Intensität des akuten Zoster- schmerzes, Unterschied zwischen den beiden zu vergleichenden Gruppen: Patienten mit und ohne vorbestehende chronische Schmerzen}

Um in den beiden zu vergleichenden Gruppen (Patienten mit und ohne vorbestehende chronische Schmerzen) Parameter ausfindig zu machen, die mit der Intensität der Zosterschmerzen in Verbindung stehen, wurde zunächst eine kumulative Schmerzintensität errechnet.

Die kumulative stärkste Schmerzintensität (NRS) ergibt sich aus der Summe der maximalen Intensität der Zosterschmerzen am Aufnahmetag, Tag 4 und Tag 7 / Entlassungstag. Die kumulative stärkste bewegungsabhängige Schmerzintensität (NRS) errechnet sich aus der Summe der maximalen bewegungsabhängigen Schmerzintensität der Zosterschmerzen am Aufnahmetag, Tag 4 und Tag 7 / Entlassungstag. Die Berechnung der kumulativen Schmerzintensität erfolgte, um die Ergebnisse übersichtlicher gestalten zu können. Für jede der beiden Gruppen wurde eine univariate Analyse durchgeführt, um Parameter ausfindig zu machen, die mit der kumulativen Schmerzintensität assoziiert waren. Die Parameter, die für die univariate Analyse verwendet wurden, sind im Kapitel 3.3.2 aufgeführt. Der dabei verwendete kumulative MQS Gesamtscore errechnet sich aus der Summe der MQS Gesamtscore für die jeweils betrachteten Tage (Aufnahmetag, Tag 4, Tag 7 / Entlassungstag). Auch hier wurden die Parameter, die sich in der univariaten Analyse als signifikant herausstellten (vgl. Tabelle 15) in die Erstellung der multivariaten Analyse mit einbezogen.

\subsubsection{Berechnung der Einflussfaktoren auf die Intensität der persistierenden Zosterschmerzen}

Im Rahmen der Langzeitbetrachtung der Patienten wurden Einflussfaktoren auf die Intensität der persistierenden Zosterschmerzen differenziert dargestellt. Als persistierende Zosterschmerzen sind in dieser Untersuchung Schmerzen (NRS $>0$ ) definiert, die im Zeitraum von drei bis sechs Monaten nach Entlassung aus der stationären Behandlung im Bereich der von Herpes Zoster befallenen Dermatome fortbestehen. Es erfolgte eine univariate Analyse (vgl. Tabelle 24) und eine multivariate Analyse (vgl. Tabelle 25) für die abhängige Variable (Outcome Parameter) ,,maximale Intensität der Zosterschmerzen“. „,Maximale Intensität der Zosterschmerzen" beschreibt die maximal aufgetretene Schmerzintensität im Zeitraum zwischen drei und sechs Monaten nach Entlassung (vgl. Kapitel 3.4.4). 


\subsubsection{Definition der Post-Zoster-Neuralgie}

Die Post-Zoster-Neuralgie wurde in der vorliegenden Studie wie folgt definiert: Maximale Intensität der Zosterschmerzen NRS $>3$, die länger als drei Monate nach der Entlassung aus der stationären Behandlung fortbestehen. Hierfür wurden die Patienten sechs Monate nach der Entlassung wie folgte befragt: „,Wie stark war der stärkste Schmerz durch Ihre Gürtelrose / Herpes Zoster in den letzten drei Monaten?“

Hierdurch ist einerseits sichergestellt, dass die Schmerzen tatsächlich länger als drei Monate bestehen. Zum anderen spiegelt die maximale Schmerzintensität im Zeitraum drei bis sechs Monate nach der Entlassung eine wesentlich längere Zeitspanne wider als beispielsweise die aktuelle Schmerzintensität zum Zeitpunkt der Nachbefragung nach drei Monaten und ist daher weniger fehleranfällig. Aus Tabelle 6 geht hervor, dass die maximale Schmerzintensität im Zeitraum drei bis sechs Monate nach der Entlassung stark mit der aktuellen Schmerzintensität zum Zeitpunkt der Nachbefragung drei Monate nach Entlassung korreliert.

Tabelle 6 Zusammenhang der gemessenen Schmerzintensitäten zu verschiedenen Zeitpunkten

\begin{tabular}{cccc} 
& Spearmans rho & $\mathbf{t}(\mathbf{n}-\mathbf{2})$ & $\mathbf{p ~}$ \\
\hline \hline $\begin{array}{l}\text { Aktuelle Schmerzintensität 6M\& } \\
\text { maximale Schmerzintensität 6M }\end{array}$ & 0,886 & 13,115 & $<0,001$ \\
$\begin{array}{c}\text { aktuelle Schmerzintensität 6M\& } \\
\text { durchschnittliche Schmerzintensität 6M }\end{array}$ & 0,920 & 16,087 & $<0,001$ \\
$\begin{array}{l}\text { maximale Schmerzintensität 6M\& } \\
\text { durchschnittliche Schmerzintensität 6M }\end{array}$ & 0,926 & 16,834 & $<0,001$ \\
$\begin{array}{l}\text { Aktuelle Schmerzintensität 3M\& } \\
\text { maximale Schmerzintensität 6M }\end{array}$ & 0,706 & & \\
\end{tabular}

\subsection{Datenschutz}

Die Erfassung der Patientendaten erfolgte pseudonymisiert. 


\section{Ergebnisse}

\subsection{Patientenkollektiv}

78 potenziell geeignete Patienten wurden im Zeitraum vom April 2012 bis zum Juni 2014 für diese Studie identifiziert. Davon waren 63 Patienten bereit, an der Studie teilzunehmen. Drei Patienten haben die Studie abgebrochen und ein Patient wurde nachträglich ausgeschlossen, da sich die Verdachtsdiagnose nicht bestätigte. Die Daten von 59 Patienten wurden ausgewertet.

$49 \%(n=29)$ der betrachteten Patienten waren Frauen, 51 \% $(n=30)$ waren Männer. Die Patienten waren zwischen 19 und 92 (68 \pm 15$)$ Jahre alt und der Body Mass Index (BMI) betrug im Durchschnitt 27,3 $\pm 4,4 \mathrm{~kg} / \mathrm{m}^{2}$. Die stationäre Behandlung betrug im Mittel 8,6 $\pm 2,2$ Tage. Der Großteil der Patienten hatte einen Volksschulabschluss (64 \%), eine Berufsausbildung (78 \%) absolviert und war zum Zeitpunkt der Untersuchung in Rente (74\%) (vgl. Tabelle 7).

Tabelle 7 Schulbildung, Ausbildung und aktuelle Tätigkeit der Patienten

\begin{tabular}{lr|lr|lr} 
Schulbildung & & Ausbildung & \multicolumn{2}{|l}{ aktuelle Tätigkeit } \\
\hline \hline keinen Schulabschluss & $3 \%$ & keine & $10 \%$ & Schule & $2 \%$ \\
Hauptschul- / Volksschulabschluss & $64 \%$ & Berufsausbildung & $78 \%$ & Arbeit & $22 \%$ \\
Realschulabschluss / Mittlere Reife & $21 \%$ & (Fach-) Hochschulstudium & $12 \%$ & Arbeitslos & $2 \%$ \\
(Fach-) Abitur & $12 \%$ & & & Rente & $74 \%$
\end{tabular}

\subsubsection{Charakterisierung der Patienten mit chronischen Schmerzen}

$48 \%$ der Patienten wiesen zum Aufnahmezeitpunkt unabhängig von den Zosterschmerzen mindestens eine chronische Schmerzentität auf. Unter Berücksichtigung der gängigen Definition von chronischen Schmerzen (Schmerzdauer > sechs Monate) hatten 42 \% der Patienten $(n=25)$ chronische Schmerzen. $58 \%$ der Patienten $(n=34)$ hatten keine vorbestehenden chronischen Schmerzen.

$\mathrm{Zu}$ Beginn der stationären Behandlung wurde die Lokalisation der chronischen Schmerzen erhoben. Dabei konnten mehrere Lokalisationen angegeben werden. Patienten mit mehr als drei Schmerlokalisationen fielen zusätzlich unter die Kategorie ,generalisiert“. Der Großteil der Patienten (40\%) hatte chronische Rückenschmerzen. Jeweils $28 \%$ gaben chronische 
Schmerzen an der Schulter und den Knien an, $20 \%$ gaben chronischen Schmerzen an den Beinen an (vgl. Abbildung 2).

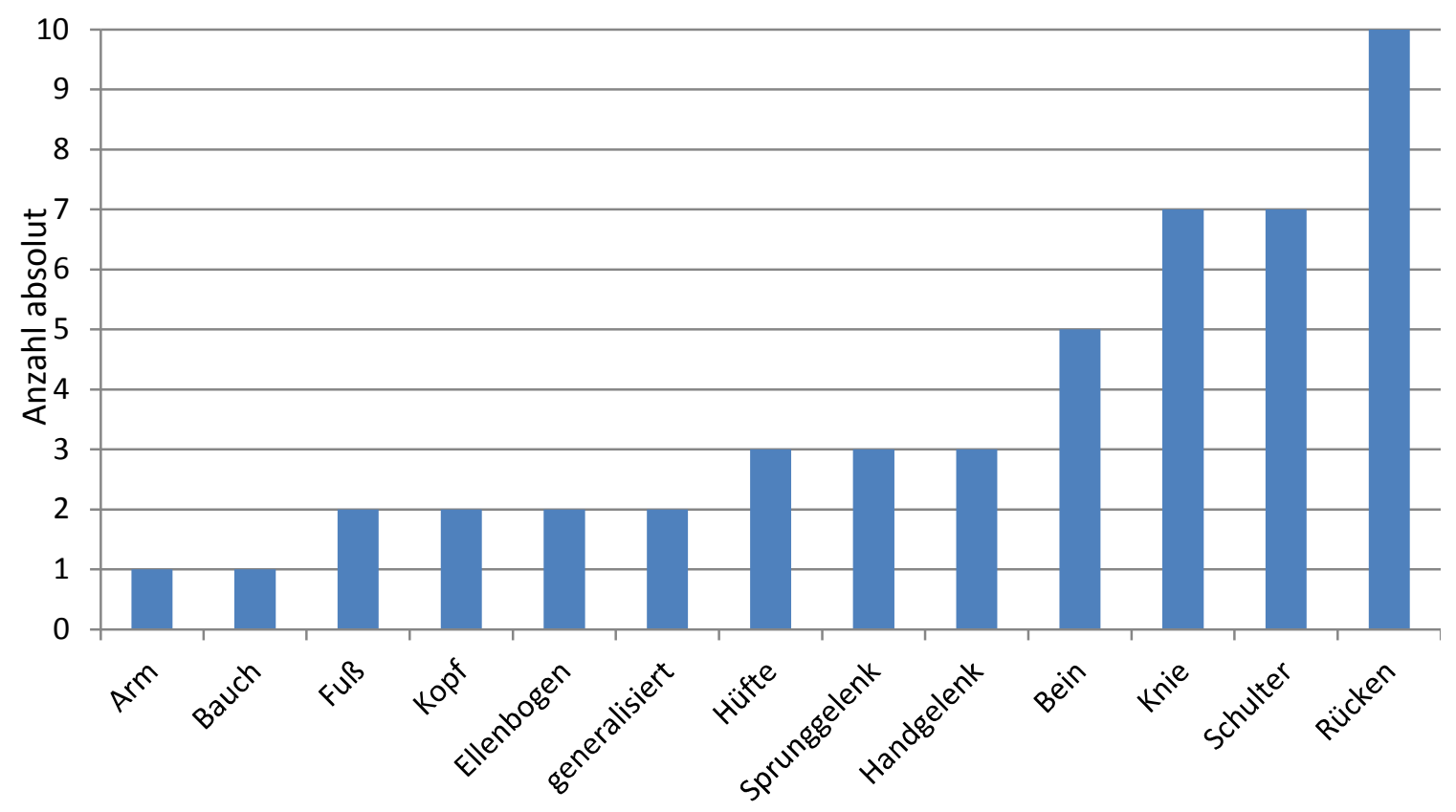

Abbildung 2 Absolute Anzahl der Schmerzlokalisationen bei Patienten mit chronischen Schmerzen. Mehrfachnennungen möglich.

$60 \%$ der Patienten mit vorbestehenden chronischen Schmerzen hatten ein Chronifizierungsstadium nach Gerbershagen (MPSS) von II. $24 \%$ befanden sich im Stadium I und $16 \%$ im Stadium III. Hinsichtlich des Schweregrades (von Korrf) der Schmerzerkrankung wurden $56 \%$ der Patienten Grad drei und vier zugeordnet, was einem dysfunktionalen chronischen Schmerz mit Beeinträchtigung der täglichen Aktivitäten durch den Schmerz entspricht (vgl. Abbildung 3).

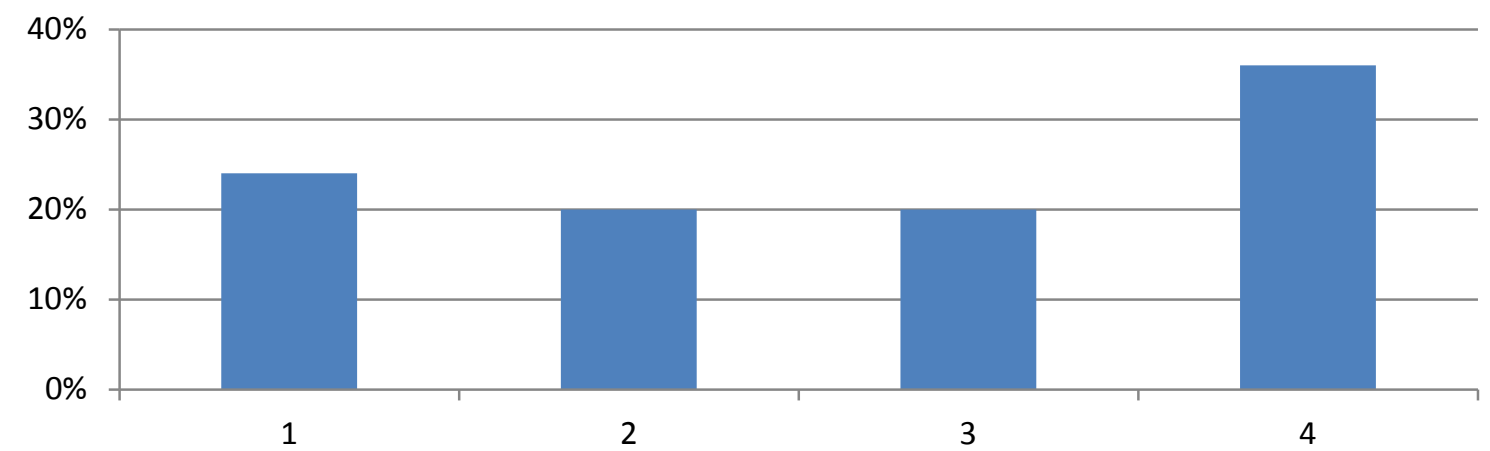

Abbildung 3 Verteilung der Chronic Pain Grade nach von Korff bei den Patienten mit vorbestehenden chronischen Schmerzen

Bei den meisten (64 \%) Patienten mit chronischen Schmerzen bestanden diese bereits länger als fünf Jahre. $20 \%$ der Patienten hatten zum Aufnahmezeitpunt 2-5 Jahre und jeweils $8 \%$ 
der Patienten hatten 1-2 Jahre bzw. 6-12 Monate chronische Schmerzen. Der Median der maximalen Intensität der chronischen Schmerzen innerhalb der letzten drei Monate vor der stationären Aufnahme betrug NRS 8 (6-9). Der Median der durchschnittlichen Intensität des chronischen Schmerzes binnen der letzten drei Monate war 4 (2,5-6).

$20 \%$ der Patienten mit chronischen Schmerzen erhielten in der Vormedikation Opioide (Transdermales Fentanyl 12 und $50 \mu \mathrm{g} / \mathrm{h}$, eine tägliche Dosis von $100 \mathrm{mg}$ Tilidin, $4 \mathrm{mg}$ Hydromorphon und $30 \mathrm{mg}$ Oxycodon). Patienten ohne chronische Schmerzen hatten keine Opioide in der Vormedikation.

\subsubsection{Charakterisierung der Zosterschmerzen}

Im Mittel wurden die Patienten 4,8 $( \pm 3,4)$ Tage nach Beginn der akuten Zosterschmerzen stationär aufgenommen. Bei $68 \%$ der Patienten war das Dermatom des Nervus Trigeminus mit einem oder mehreren seiner Äste betroffen (vgl. Abbildung 4).

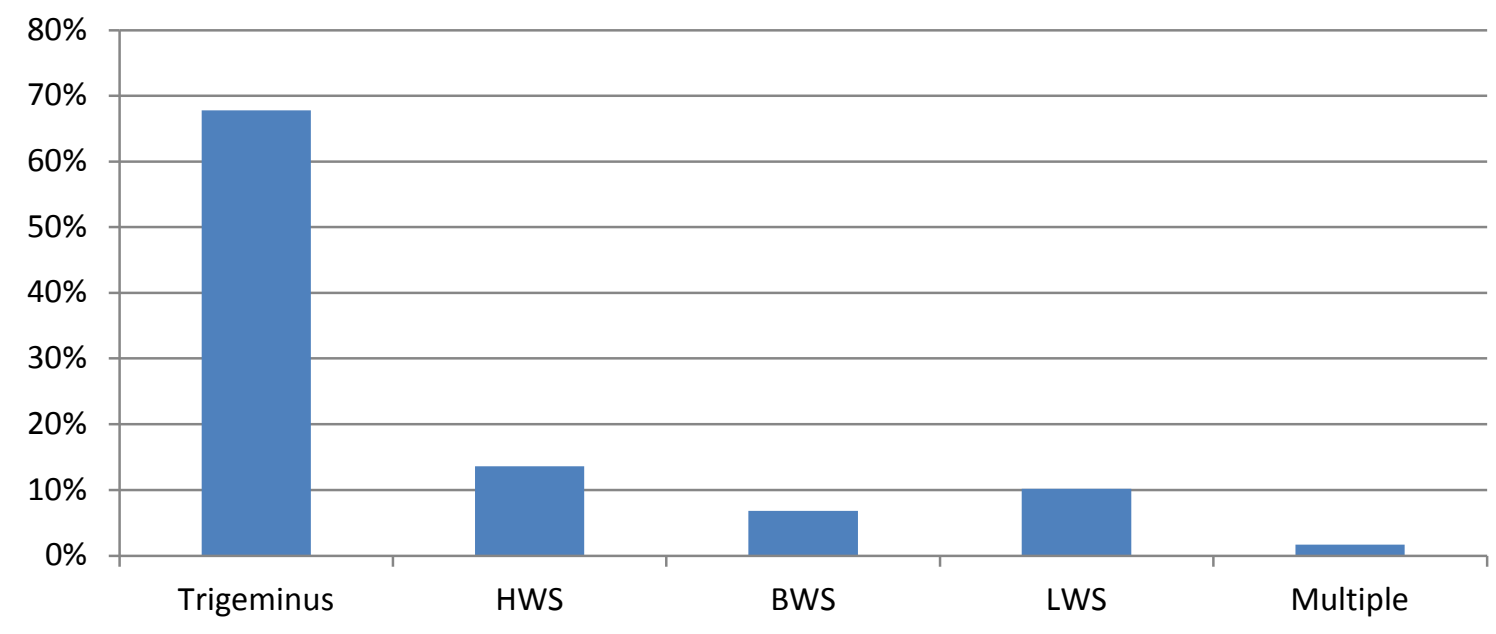

Abbildung 4 Betroffene Dermatome der Herpes-Zoster-Infektion

Mit dem Deutschen Schmerzfragebogen wurde unter anderem anhand von vorgegebenen Adjektiven die Beschreibung der Zosterschmerzen bei Aufnahme untersucht. $81 \%$ beschrieben die Schmerzen als stechend. Jeweils ca. 70 \% der Patienten empfanden den Schmerz als brennend und ziehend (vgl. Abbildung 5). 


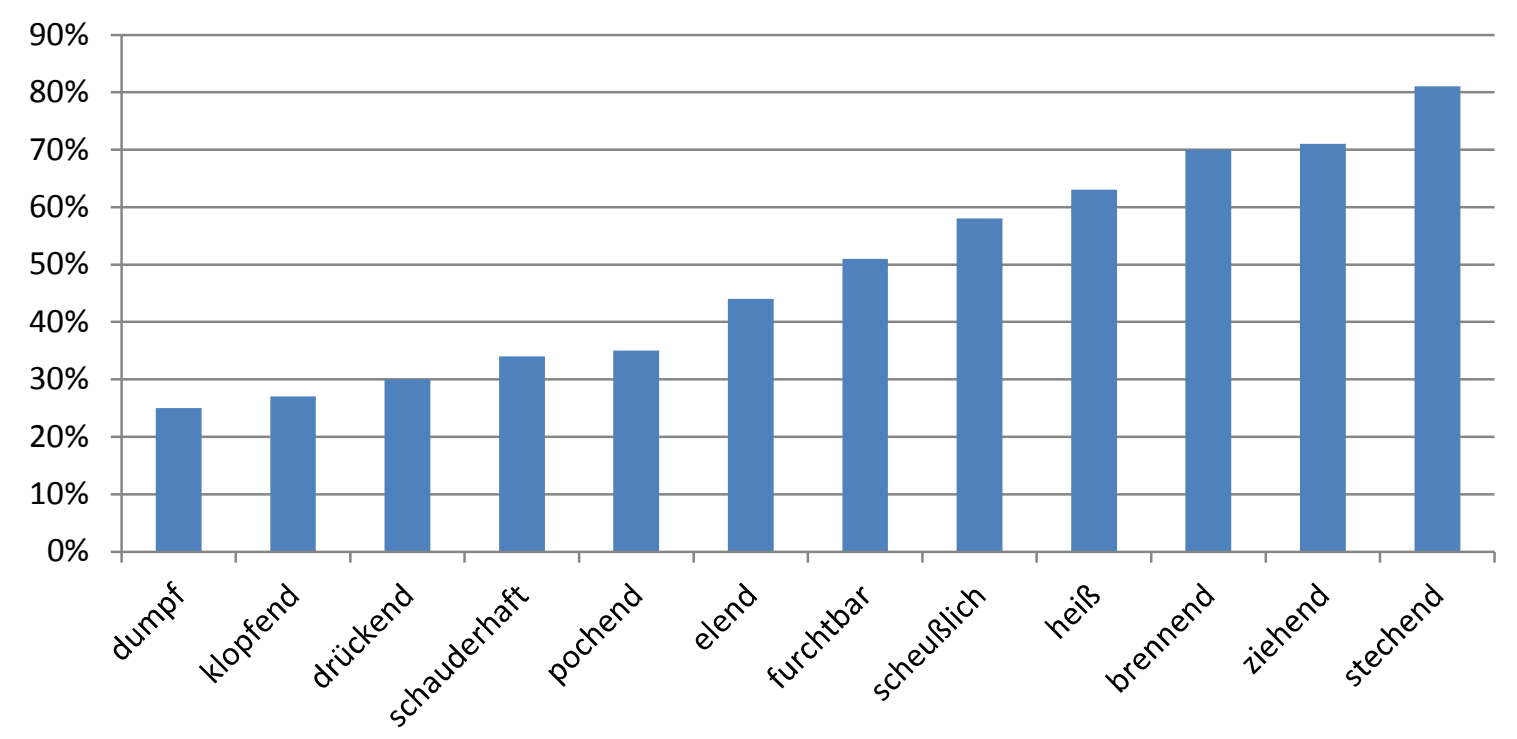

Abbildung 5 Beschreibung der Zosterschmerzen

Insgesamt beschrieben $68 \%$ der an Zoster erkrankten Personen ein durch Schmerzattacken charakterisiertes Schmerzbild. Bei 14 \% der Patienten unterlagen die Schmerzen starken Schwankungen und bei $18 \%$ leichten Schwankungen im Tagesverlauf.

Für 48 \% der Patienten wurde das Vorhandensein von neuropathischen Schmerzkomponenten (painDETECT) als ,unwahrscheinlich“ eingestuft. Als „,wahrscheinlich neuropathisch“ wurden die Schmerzen von 27 \% der Patienten eingeordnet. Bei $25 \%$ der Patienten war beim Screening eine neuropathische Schmerzkomponente nicht eindeutig darzustellen.

\subsubsection{Verlauf der Intensität der Zosterschmerzen während der stationären Be- handlung}

Der Median der maximale Schmerzintensität betrug am Aufnahmetag NRS 5 (3-8) und reduzierte sich im Verlauf auf NRS 3 (3-4) an Tag 7 / Entlassungstag (vgl. Abbildung 6). Auch die Intensität der Zosterschmerzen bei Belastung reduzierte sich bei den Patienten von NRS 4 (2-7) am Aufnahmetag auf NRS 2 (0-3) an Tag 7 / Entlassungstag. 


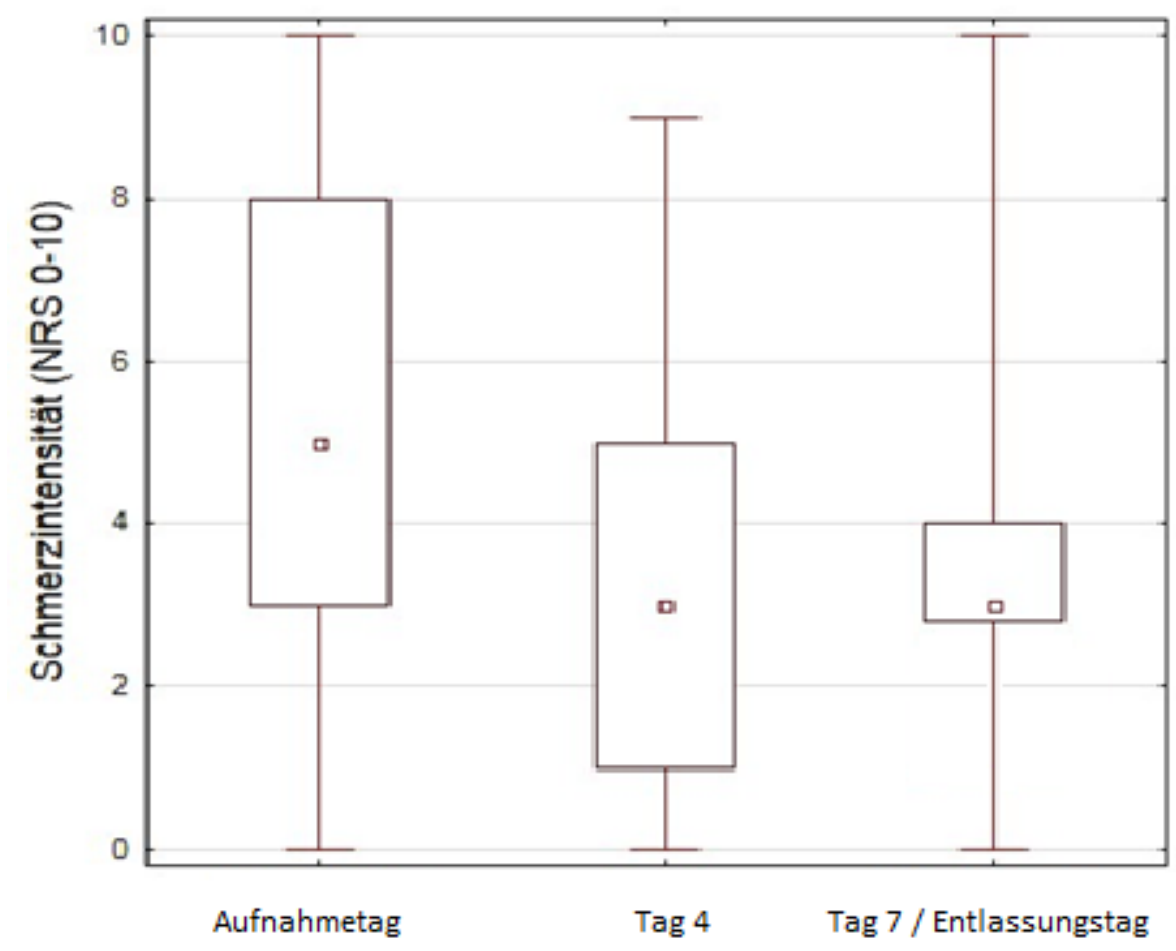

Abbildung 6 Maximale Intensität der Zosterschmerzen im Verlauf der stationären Behandlung. In dem Box-Plot ist jeweils dargestellt der Median (mit Quartile und Extremwerten) der maximalen Schmerzintensität (NRS).

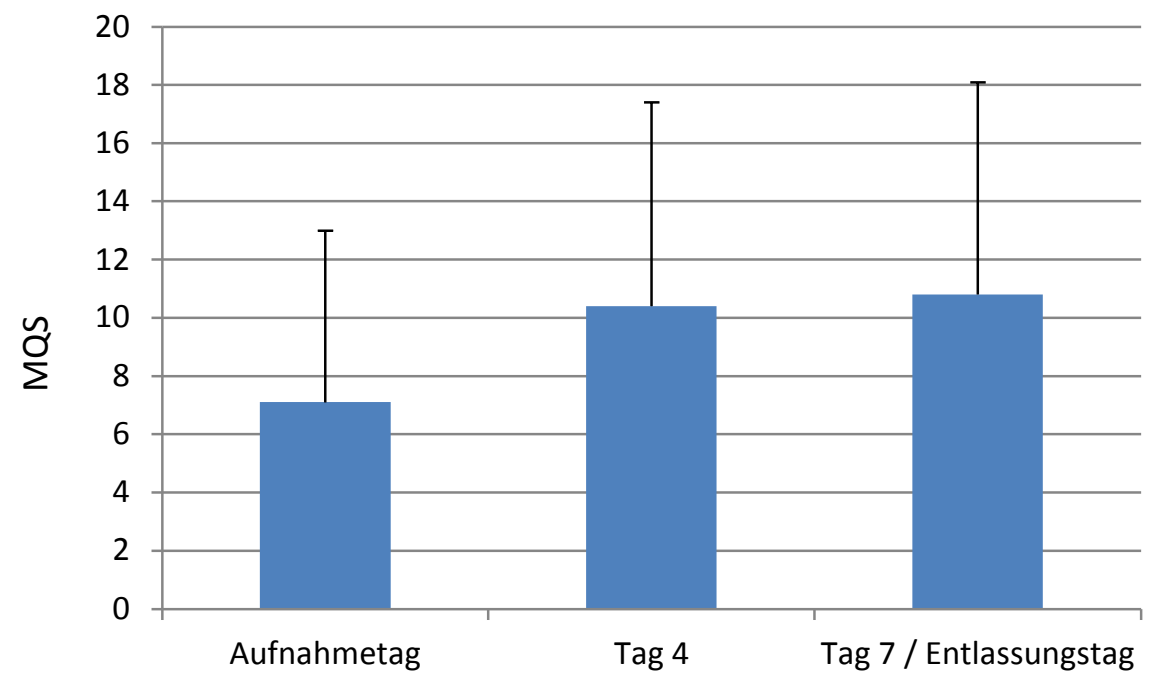

Abbildung 7 Schmerzmedikation (MQS) im Verlauf der stationären Behandlung. Dargestellt ist der Mittelwert (mit Standardabweichung) des MQS-Gesamtscores. 
Im Rahmen der analgetischen Behandlung erhielt rund die Hälfe der Patienten (49 \%) Opioide, $88 \%$ der Patienten wurden mit Nicht-Opioiden therapiert und $53 \%$ der Patienten erhielten Co-Analgetika (39\% Antikonvulsiva, 14 \% Antidepressiva). In Abbildung 7 sind die verabreichten Schmerzmedikamente (MQS) im Verlauf der stationären Behandlung dargestellt. Die antivirale Therapie wurde während der stationären Behandlung bei allen Patienten mittels intravenös applizierten Aciclovir (5 bis 10 mg/kg Körpergewicht alle 8 Stunden) durchgeführt.

\subsubsection{Psychologische Charakterisierung bei Aufnahme}

Im Zuge dieser Studie wurden auch psychologische Charakteristika der Patienten ermittelt. Nachfolgend sind die Ergebnisse aller an der akuten Herpes-Zoster-Infektion erkrankten Patienten deskriptiv dargestellt.

Anhand der Depression Anxiety Stress Scale (DASS) wurden die Patienten ermittelt, die die jeweiligen Cut-Off-Werte für Depression, Angst und Stress überschritten hatten. 80 \% der Patienten hatten Werte unterhalb der jeweiligen Cut-off-Werte und waren keiner der genannten Störungen zuzuordnen. 3 \% $(n=2)$ der Patienten hatten eine erhöhte Wahrscheinlichkeit für das Vorliegen einer depressiven Störung. Jeweils $5 \%(n=3)$ der Patienten hatten die CutOff-Werte für Angst bzw. Stress überschritten. Bei weiteren $5 \%(n=3)$ der Patienten lagen Hinweise auf eine Kombination von Depression, Angst und Stress vor. Bei $2 \%$ der Patienten $(n=1)$ bestanden Hinweise auf das Vorliegen einer Kombination aus Despression und Stress.

Mit dem PHQ wurde für 33 \% der Patienten eine geringe Somatisierungstendenz aufgezeigt. Bei $60 \%$ der Patienten wurden Werte unterhalb der jeweiligen Cut-off-Werte ermittelt und daher keine Tendenz zur Somatisierung nachgewiesen (vgl. Abbildung 8).

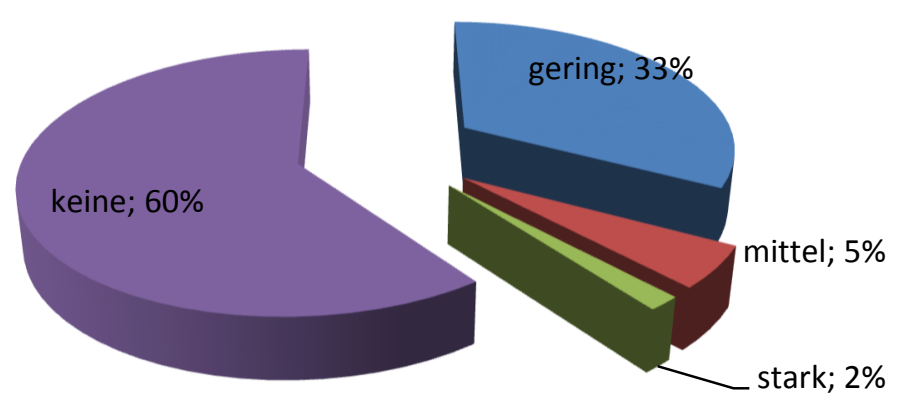

Abbildung 8 Somatisierungstendenz (PHQ) 
Weitere psychologische Charakteristika für den Aufnahmetag lassen sich aus Tabelle 8 entnehmen.

Tabelle 8 Psychologische Charakteristika für den Aufnahmetag

Merkmal

Mittelwert

Bewegungsbezogene Angst (TSK)

$39,6 \pm 7,5$

Hilfs- und Hoffnungslosigkeit (KPI)

$1,7 \pm 1,1$

Katastrophisieren (KPI)

$2,2 \pm 1,1$

Durchhalteappell (KPI)

$2,9 \pm 1,4$

Gesundheitsbezogene Lebensqualität (SF 12):

-Körperliche Gesundheit

$38,9 \pm 10,9$

-Psychische Gesundheit

$47,0 \pm 10,1$

\subsection{Vergleich der Patienten mit und ohne vorbestehende chronische Schmerzen}

Ein Ziel dieser Studie war es, die Unterschiede hinsichtlich des klinischen Verlaufes zwischen Patienten mit vorbestehenden chronischen Schmerzen und ohne chronische Schmerzen zu untersuchen. Dazu wurden die Patienten in zwei Gruppen aufgeteilt. Der Gruppe „chronische Schmerzen“ (chronic pain; CP) wurden die Patienten mit zusätzlich zu dem akuten Zosterschmerz vorbestehenden chronischen Schmerzen zugeordnet. Patienten ohne vorbestehende chronische Schmerzen fielen in die Gruppe „keine chronischen Schmerzen“ (non-chronic pain; nCP). Der Gruppe CP wurden insgesamt 25 Patienten zugeordnet, 13 Frauen und 12 Männer. In der Gruppe nCP befanden sich 34 Patienten (16 Frauen und 18 Männer) (vgl. Abbildung 9).

Der Unterschied in der Verteilung von Frauen und Männern zwischen den Gruppen war nicht signifikant $\left(p=0,446, X^{2}=0,141\right)$.

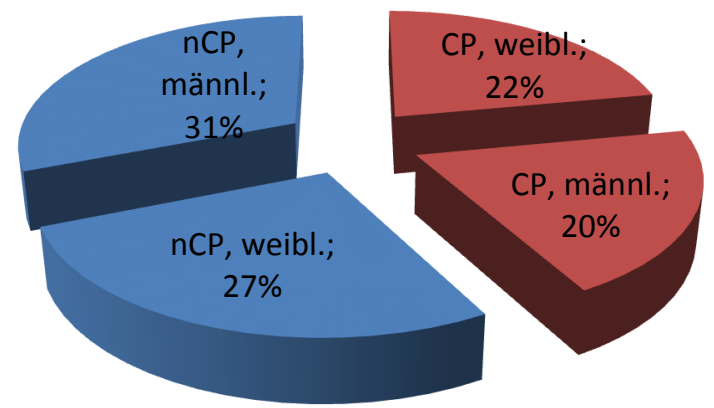

Abbildung 9 Verteilung der Geschlechter in den Gruppen nCP und CP 
Die Patienten mit chronischen Schmerzen waren signifikant älter als Patienten ohne chronische Schmerzen (CP: 74,2 $\pm 11,3$ Jahre; nCP: 63,9 $\pm 16,2$ Jahre; $\mathrm{p}=0,008, \mathrm{t}=-2,739$ ). Der Body Mass Index (BMI) unterschied sich nicht signifikant (CP 26,8 $\pm 4,2$, nCP 27,7 $\pm 4,5 ; \mathrm{p}=0,455$, $\mathrm{t}=0,753)$.

\subsubsection{Psychologische Charakterisierung}

Die gesundheitsbezogene Lebensqualität (SF 12) war bei den Patienten mit chronischen Schmerzen signifikant vermindert. Dieses gilt sowohl für die körperliche als auch für die psychische Gesundheit (vgl. Abbildung 10).

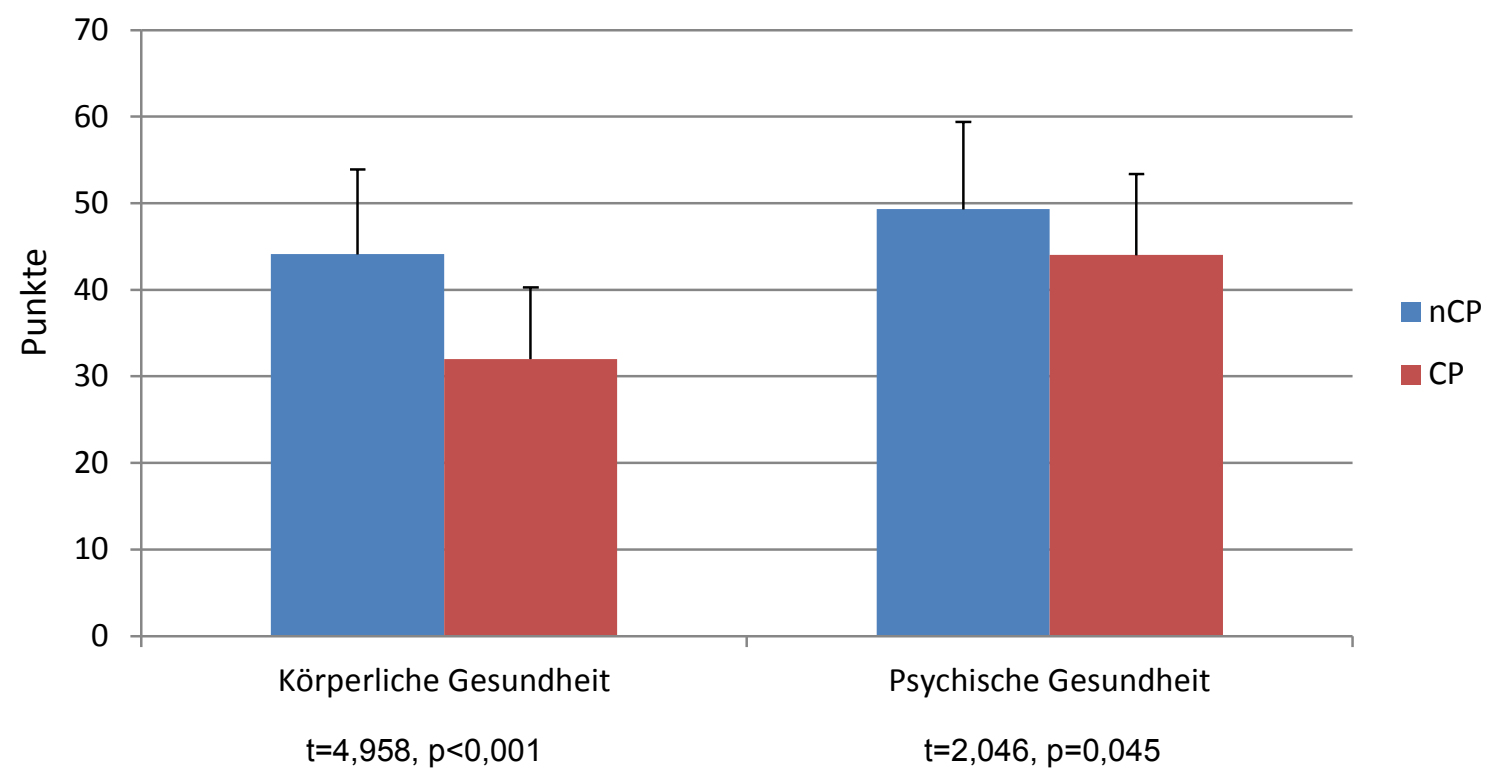

Abbildung 10 Körperliche und psychische Lebensqualität (SF 12). Dargestellt ist der Mittelwert (mit Standardabweichung).

Bezüglich der Werte für Depression, Angst und Stress konnte zum Aufnahmezeitpunkt kein signifikanter Unterschied gezeigt werden (vgl. Tabelle 9). Ebenfalls keinen signifikanten Unterschied gab es zum Aufnahmetag in der Anzahl der Patienten, welche die jeweiligen Cut-Off-Werte überschritten hatten (Depression: $\mathrm{nCP}=15 \%, \mathrm{CP}=4 \%$; Angst: $\mathrm{nCP}=6 \%$, $\mathrm{CP}=16 \%$; Stress: $\mathrm{nCP}=15 \%, \mathrm{CP}=8 \%$ ). Patienten der Gruppe $\mathrm{CP}$ hatten allerdings am Ende des stationären Krankenhausaufenthaltes signifikant höhere Werte für Stress und Angst (vgl. Abbildung 11). 


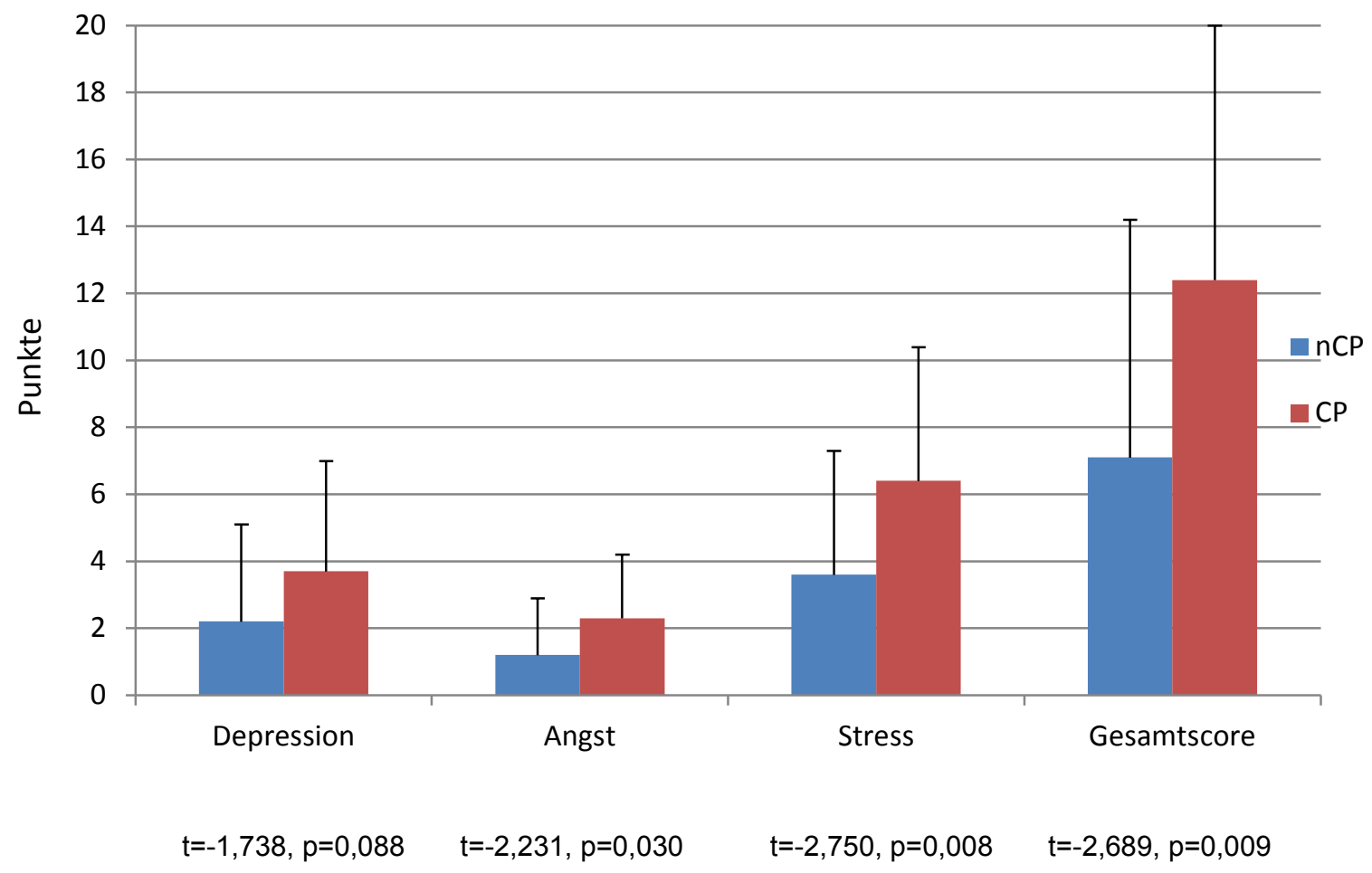

Abbildung 11 Depression, Angst und Stress während der stationären Behandlung, Erhebung am Entlassungstag. Dargestellt ist der Mittelwert (mit Standardabweichung).

Tabelle 9 Depression, Angst und Stress zum Aufnahmezeitpunkt

\begin{tabular}{lcccc} 
Parameter & $\mathbf{C P}$ & $\mathbf{n C P}$ & $\mathbf{t}$ & $\mathbf{p}$ \\
\hline \hline Depression & $4,4 \pm 4,0$ & $3,9 \pm 4,4$ & $-0,545$ & 0,588 \\
Angst & $3,0 \pm 2,6$ & $2,0 \pm 2,3$ & $-1,588$ & 0,118 \\
Stress & $5,9 \pm 4,0$ & $4,6 \pm 4,8$ & $-1,074$ & 0,287
\end{tabular}

Patienten mit chronischen Schmerzen zeigten bei Aufnahme ein signifikant häufigeres Auftreten von „Hilf- und Hoffnungslosigkeit“ als Schmerzverarbeitungsmuster (vgl. Abbildung 12). 


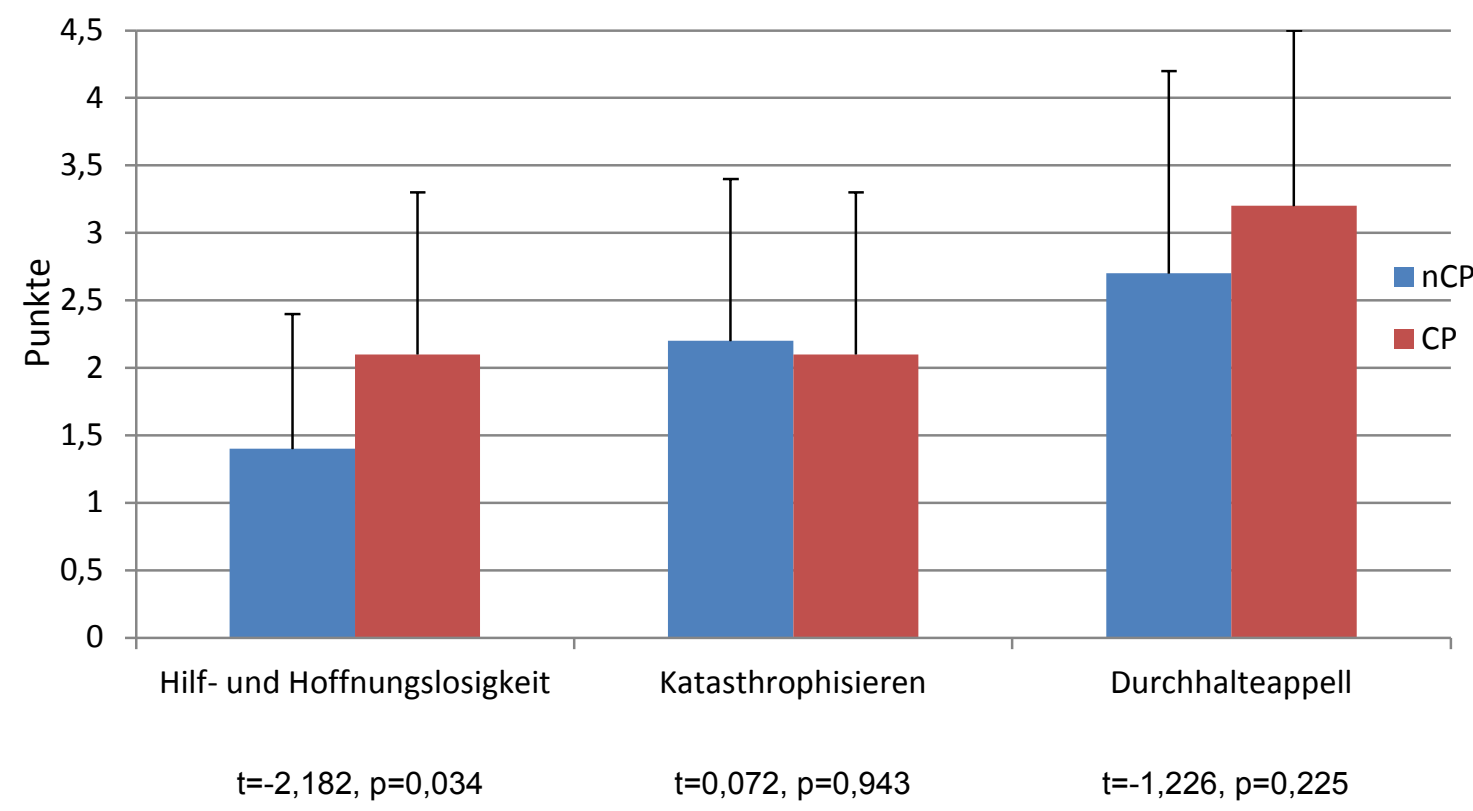

Abbildung 12 Schmerzverarbeitungsmuster (KPI). Dargestellt ist der Mittelwert (mit Standardabweichung).

Patienten mit chronischen Schmerzen hatten des Weiteren eine signifikant höhere bewegungsbezogene Angst (TSK) zum Aufnahmezeitpunkt (nCP: 37,3 $\pm 6,3$ Punkte; CP: 42,6 \pm 8 $\mathrm{t}=-2,793, \mathrm{p}=0,007$ ) und zum Zeitpunkt der Entlassung (nCP: 36,1 $\pm 7,1$ Punkte; CP: 42,2 $\pm 8 ; 8 \mathrm{t}=-2,793, \mathrm{p}=0,006)$. Außerdem zeigten CP-Patienten sowohl am Aufnahmetag als auch am Entlassungstag eine signifikant höhere Tendenz zur Somatisierung (vgl. Abbildung 13).

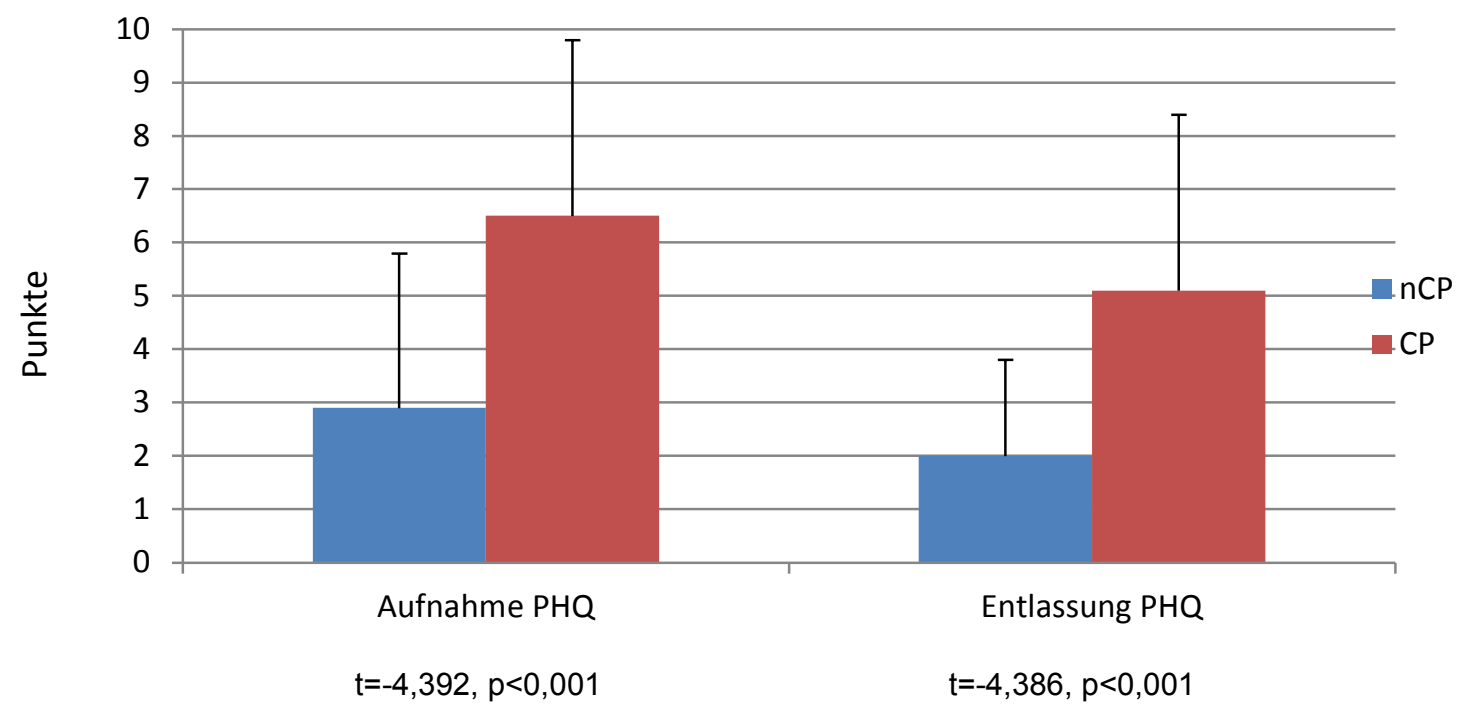

Abbildung 13 Somatisierungstendenz (PHQ) am Aufnahme- und Entlassungstag. Dargestellt ist der Mittelwert (mit Standardabweichung). 


\subsubsection{Charakterisierung der Zosterschmerzen}

Patienten mit chronischen Schmerzen hatten an allen Tagen der Erhebung eine signifikant höhere Intensität der durch Zoster verursachten Schmerzen als Patienten ohne vorbestehende chronische Schmerzen (vgl. Abbildung 14).

a) Maximale Schmerzintensität

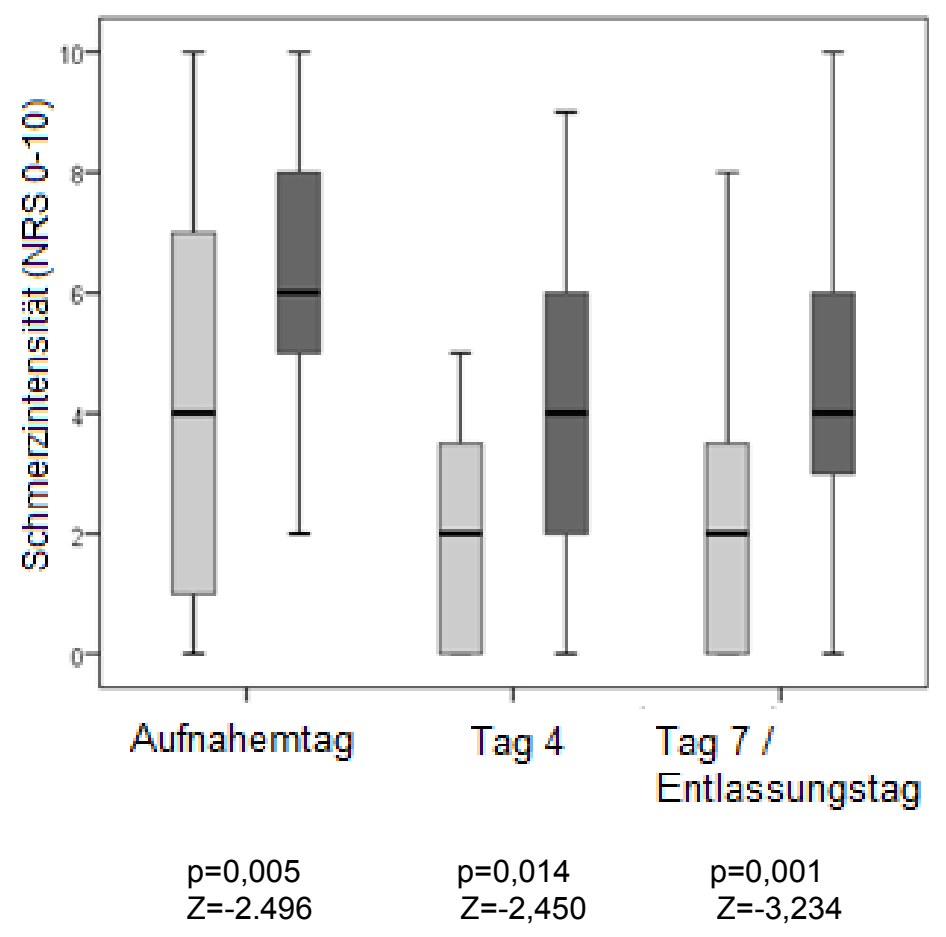

b) Minimale Schmerzintensität

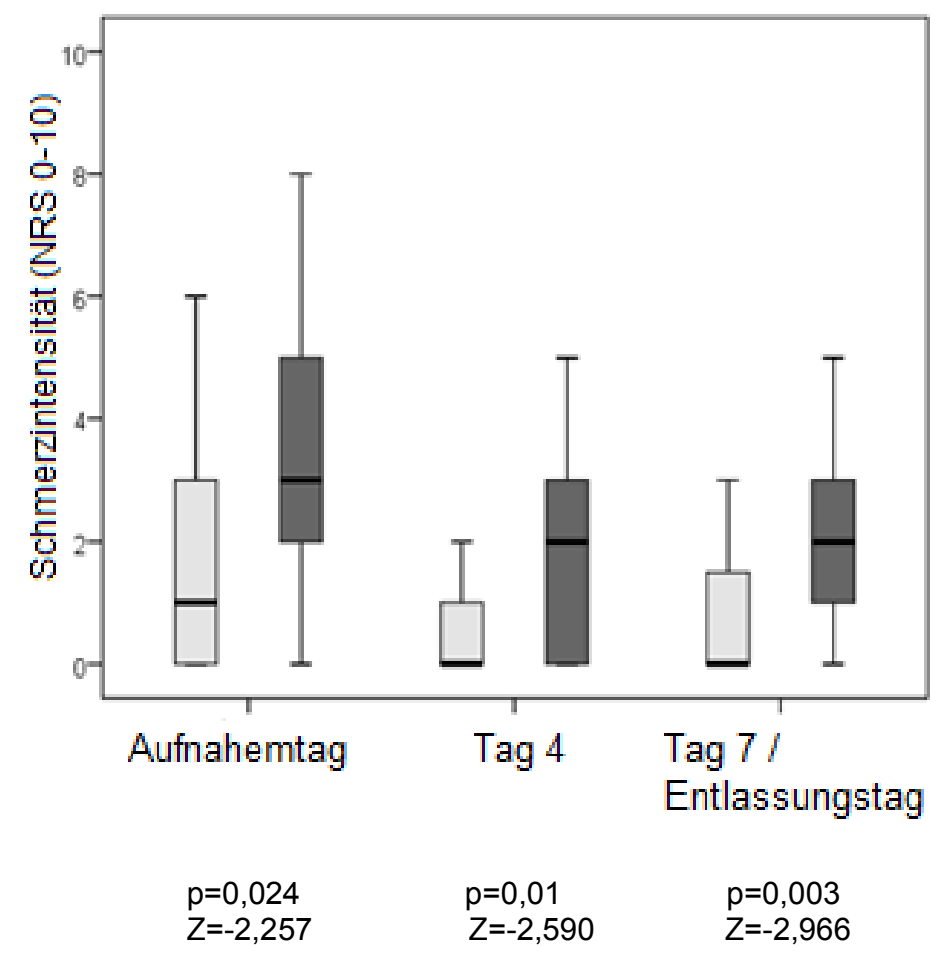




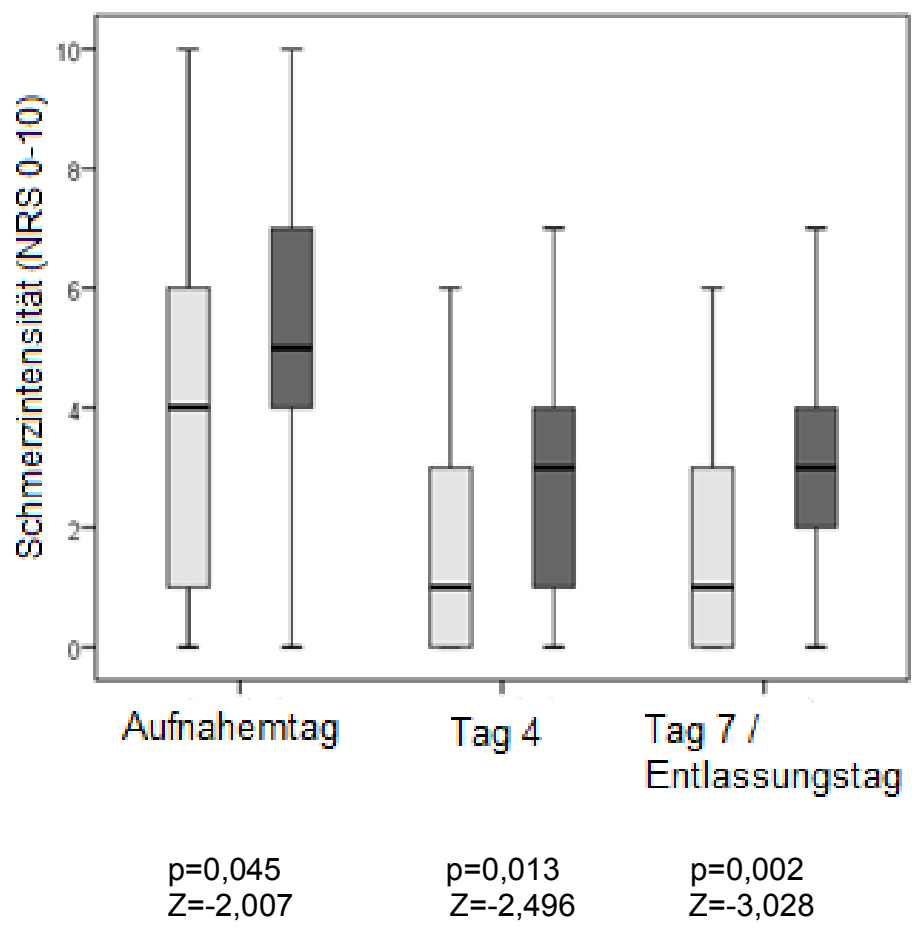

Abbildung 14 Intensität der Zosterschmerzen im Verlauf. Graue Balken: nCP; schwarze Balken: CP. In dem Box-Plots sind jeweils dargestellt der Median (mit Quartile und Extremwerten) der Schmerzintensität (NRS).

Es gab am Aufnahmetag und am Entlassungstag keinen signifikanten Unterschied hinsichtlich des Vorhandenseins von neuropathischen Schmerzkomponenten (painDETECT) (vgl. Tabelle 10).

Tabelle 10 Ergebnisse des painDETECT

Parameter

\begin{tabular}{ccccc}
\hline PainDETECT Aufnahmetag & $15,6 \pm 8,9$ & $11,6 \pm 7,3$ & $-1,906$ & 0,062 \\
& & & & \\
PainDETECT Entlassungstag & $11,9 \pm 6,9$ & $8,3 \pm 7,2$ & $-1,927$ & 0,059
\end{tabular}

Es zeigt sich kein signifikanter Unterschied in der Dauer der Zosterschmerzen bis zur stationären Aufnahme (CP: 5 (3-7) Tage, nCP: 5 (3-5) Tage; $Z=-1,600, p=0,110)$. Patienten mit chronischen Schmerzen hatten jedoch einen signifikant längeren stationären Aufenthalt (CP: $9(8-10,5)$ Tage, $n C P: 8(6,25-9)$ Tage; $p=0,021, Z=-2,302)$. 


\subsubsection{Beeinträchtigungen während des Aufenthaltes}

Mittels einer modifizierten Version des QUIPS wurden sowohl die Beeinträchtigungen der Patienten durch die Schmerzen als auch die Beeinträchtigung durch weitere Symptome (Vorhandensein von Müdigkeit, Übelkeit und Erbrechen) untersucht. Dabei zeigte sich, dass Patienten mit vorbestehenden chronischen Schmerzen während der stationären Behandlung durch die Schmerzen in ihrer Stimmung beeinträchtigt waren. Ferner zeigte sich bei den Patienten der Gruppe CP an zwei erhobenen Zeitpunkten im Trend eine Beeinträchtigung in der Mobilität durch die Schmerzen (vgl. Tabelle 11).

Tabelle 11 Beeinträchtigungen während des stationären Aufenthalts

\begin{tabular}{|c|c|c|c|c|c|}
\hline Fragestellung & & $\underset{*}{\text { Aufn. }}$ & Tag 4 & $\begin{array}{c}\text { Tag } 7 \text { I } \\
\text { Entl. } \\
\star \star \star\end{array}$ & Statistik \\
\hline $\begin{array}{l}\text { Sind Sie durch die Schmerzen in Ihrer } \\
\text { Mobilität bzw. Bewegung beeinträchtigt? } \\
\text { [,,ja“] }\end{array}$ & $\begin{array}{l}\mathrm{CP} \\
\mathrm{nCP}\end{array}$ & $\begin{array}{l}60 \\
27\end{array}$ & 24 & $\begin{array}{l}28 \\
9\end{array}$ & $\begin{array}{l}p=0,01, X^{2}=6,712^{*} \\
p=0,026, X^{2}=5,461 \text { ** } \\
p=0,087, X^{2}=3,366 \text { *** }\end{array}$ \\
\hline $\begin{array}{l}\text { Sind Sie durch die Schmerzen beim } \\
\text { Husten oder tiefen Luft holen beein- } \\
\text { trächtigt? [,ja“] }\end{array}$ & $\mathrm{CP}$ & $\begin{array}{l}12 \\
24\end{array}$ & $\begin{array}{l}12 \\
0\end{array}$ & $\begin{array}{l}12 \\
3\end{array}$ & $\begin{array}{l}p=0,297, X^{2}=1,532 * \\
p=0,083, X^{2}=3,931 * * \\
p=0,309, X^{2}=1,694 * *\end{array}$ \\
\hline $\begin{array}{l}\text { Sind Sie durch die Schmerzen heute } \\
\text { Nacht aufgewacht? [,,ja“] }\end{array}$ & $\mathrm{CP}$ & $\begin{array}{l}68 \\
35\end{array}$ & 44 & 36 & $\begin{array}{l}p=0,013, X^{2}=6,166^{*} \\
p=0,023, X^{2}=5,268^{* *} \\
p=0,022, X^{2}=5,986 \text { *** }\end{array}$ \\
\hline $\begin{array}{l}\text { Sind Sie durch die Schmerzen in der } \\
\text { Stimmung beeinträchtigt? [,,ja“] }\end{array}$ & $\begin{array}{l}\mathrm{CP} \\
\mathrm{nCP}\end{array}$ & $\begin{array}{l}80 \\
44\end{array}$ & $\begin{array}{l}64 \\
16\end{array}$ & $\begin{array}{l}60 \\
6\end{array}$ & $\begin{array}{l}p=0,005, X^{2}=7,687^{*} \\
p<0,001, X^{2}=13,532 \text { ** } \\
p<0,001, X^{2}=19,374 \text { *** }\end{array}$ \\
\hline $\begin{array}{l}\text { Hätten Sie sich gewünscht, mehr Mittel } \\
\text { gegen Schmerzen zu bekommen? [,,ja“] }\end{array}$ & $\begin{array}{l}\mathrm{CP} \\
\mathrm{nCP}\end{array}$ & $\begin{array}{l}32 \\
18\end{array}$ & 16 & 16 & 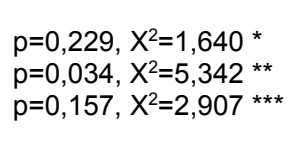 \\
\hline $\begin{array}{l}\text { Haben Sie sich heute sehr müde ge- } \\
\text { fühlt? [,ja“] }\end{array}$ & $\begin{array}{l}\mathrm{CP} \\
\mathrm{nCP}\end{array}$ & $\begin{array}{l}76 \\
56\end{array}$ & $\begin{array}{l}56 \\
39\end{array}$ & $\begin{array}{l}64 \\
22\end{array}$ & $\begin{array}{l}p=0,169, X^{2}=2,543 * \\
p=0,282, X^{2}=1,663 \text { ** } \\
p=0,001, X^{2}=10,348 \text { *** }\end{array}$ \\
\hline $\begin{array}{l}\text { Haben Sie heute unter Übelkeit gelitten? } \\
\text { [,ja“] }\end{array}$ & $\begin{array}{l}\mathrm{CP} \\
\mathrm{nCP}\end{array}$ & $\begin{array}{l}20 \\
12\end{array}$ & $\begin{array}{l}12 \\
10\end{array}$ & $\begin{array}{l}24 \\
3\end{array}$ & $\begin{array}{l}p=0,474, X^{2}=0,756 * \\
p=0,556, X^{2}=0,078 * * \\
p=0,037, X^{2}=5,461 * * *\end{array}$ \\
\hline
\end{tabular}




\subsubsection{Schmerztherapie}

Während der stationären Behandlung bestand kein signifikanter Unterschied bezüglich der Menge der applizierten Analgetika zwischen den beiden Gruppen (vgl. Abbildung 15). Der Analgetikakonsum wurde mit Hilfe des MQS quantifiziert. In diesen Score gingen Opioide, Nicht-Opioide und Co-Analgetika (Antikonvulsiva und Antidepressiva) ein.

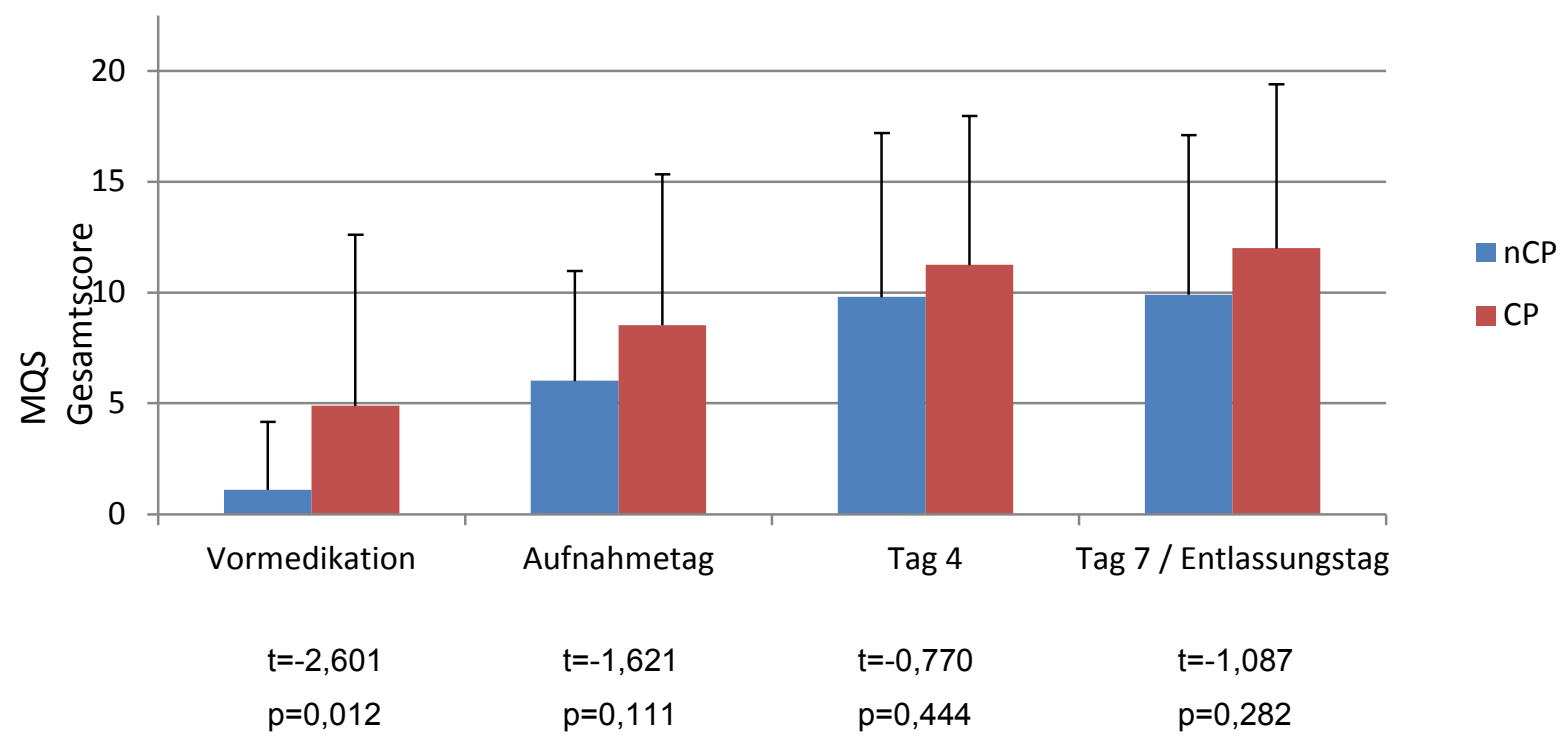

Abbildung 15 Analgetikakonsum (MQS Gesamtscore). Dargestellt ist der Mittelwert (mit Standardabweichung) des MQS Gesamtscores.

Des Weiteren zeigte sich kein signifikanter Unterschied in der Applikation der verschiedenen Analgetika-Gruppen (vgl. Tabelle 12). Patienten mit vorbestehenden chronischen Schmerzen erhielten allerdings mehr Opioide (in mg Morphinäquivalent). Bei der Betrachtung der Opioide (in mg Morphinäquivalent), die die Patienten zusätzlich zu ihrer Vormedikation im Rahmen der Therapie des Herpes Zoster erhielten, bestand kein signifikanter Unterschied zwischen den beiden Gruppen. 
Tabelle 12 Analgetikakonsum während der stationären Behandlung

\begin{tabular}{l|ccc} 
& nCP & CP & Statistik \\
\hline \hline Analgetika ( \%) & & & \\
Nicht-Opioide & 85 & 92 & $\mathrm{p}=0,447, \mathrm{X}^{2}=1,144$ \\
Opioide & 41 & 60 & $\mathrm{p}=0,605, \mathrm{X}^{2}=0,359$ \\
Antikonvulsiva & 41 & 36 & $\mathrm{p}=0,790, \mathrm{X}^{2}=0,162$ \\
Antidepressiva & 6 & 24 & $\mathrm{p}=0,122, \mathrm{X}^{2}=2,746$ \\
\hline Opioide (ME in mg) & & & \\
Aufnahmetag & $2,9 \pm 4,3$ & $15,8 \pm 28,5$ & $\mathrm{p}=0,013, \mathrm{t}=-2,573$ \\
Tag 4 & $5,8 \pm 8,2$ & $17,3 \pm 28,2$ & $\mathrm{p}=0,030, \mathrm{t}=-2,216$ \\
Tag 7 / Entlassungstag & $7,4 \pm 16,8$ & $18,2 \pm 27,7$ & $\mathrm{p}=0,074, \mathrm{t}=-1,819$ \\
\hline $\begin{array}{l}\text { Opioide zusätzlich zur } \\
\text { Vormedikation (ME in mg) }\end{array}$ & & & $\mathrm{p}$ \\
Aufnahmetag & $2,9 \pm 4,3$ & $2,4 \pm 8,7$ & $\mathrm{p}=0,901, \mathrm{t}=0,126$ \\
Tag 4 & $6,0 \pm 8,3$ & $3,9 \pm 19,2$ & $\mathrm{p}=0,572, \mathrm{t}=0,568$ \\
Tag 7 / Entlassungstag & $7,2 \pm 16,6$ & $4,8 \pm 19,1$ & $\mathrm{p}=0,614, \mathrm{t}=0,507$ \\
& & &
\end{tabular}

Patienten der Gruppe CP waren zu allen betrachteten Zeitpunkten signifikant weniger zufrieden mit der Schmerztherapie als Patienten der Gruppe nCP (vgl. Tabelle 13).

Tabelle 13 Zufriedenheit mit der Schmerztherapie (NRS 0-15)

\begin{tabular}{ccccc} 
Zeitpunkt & CP & nCP & Z & p \\
\hline \hline Aufnahmetag & $13(12-14)$ & $14,5(13,75-15)$ & 2,454 & 0,014 \\
Tag 4 & $14(11,5-14)$ & $15(14-15)$ & 3,511 & $<0,001$ \\
Tag 7 / Entlassungstag & $14(13-15)$ & $15(14-15)$ & 2,518 & 0,012
\end{tabular}




\subsubsection{Druckschmerzschwelle}

Patienten mit chronischen Schmerzen hatten zum Zeitpunkt der Aufnahme eine signifikant geringere Druckschmerzschwelle (vgl. Abbildung 16).

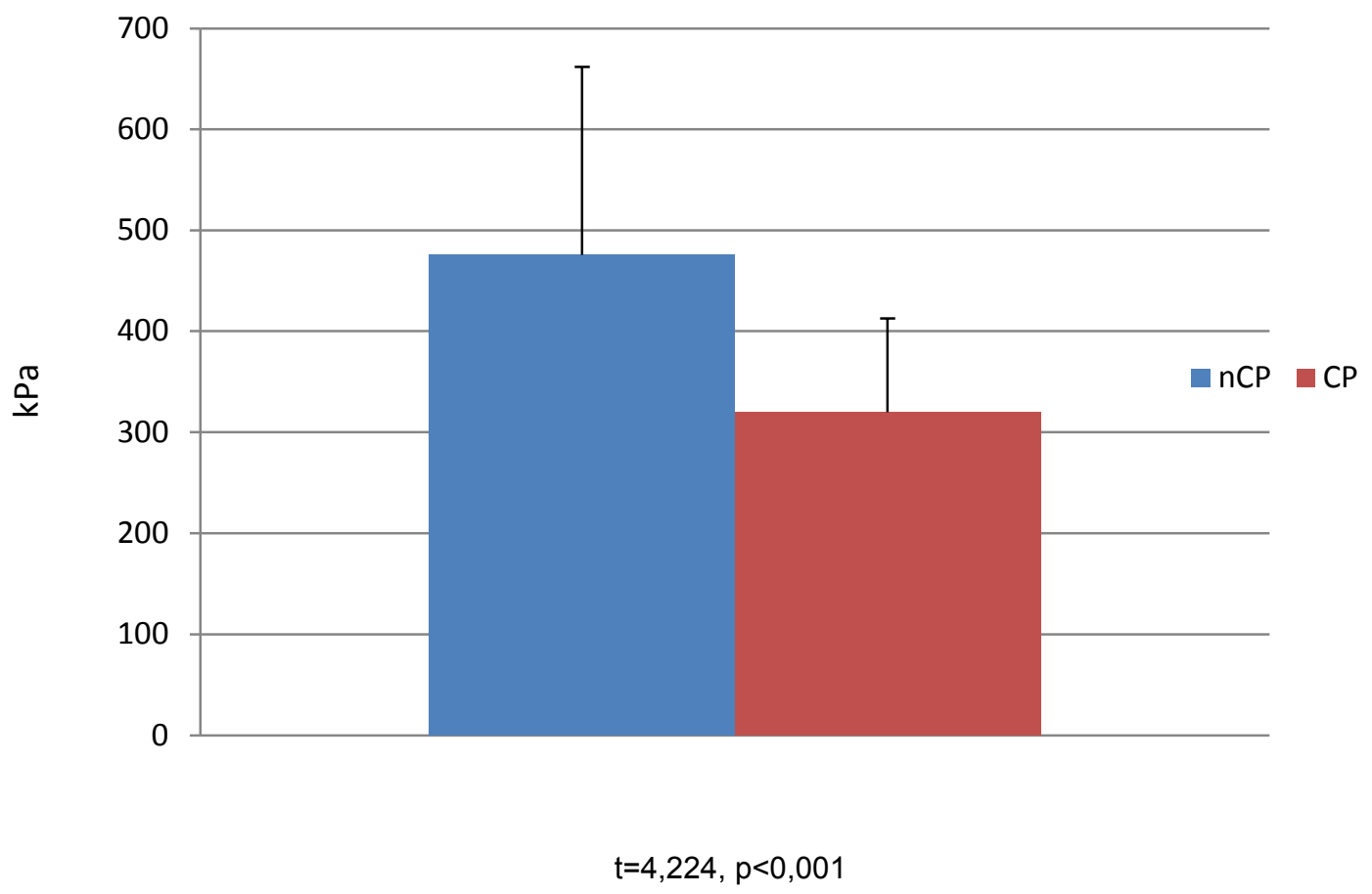

Abbildung 16 Mittelwert und Standardabweichung der Druckschmerzschwelle bei den Patienten mit und ohne vorbestehende chronische Schmerzen

\subsection{Einflussfaktoren auf die Intensität des akuten Zosterschmerzes}

\subsubsection{Bezogen auf das gesamte Patientenkollektiv}

In diesem Abschnitt wird dargestellt, welche Faktoren Einfluss auf die maximale Intensität des akuten Zosterschmerzes (QUIPS) hatten. Zunächst wurde für den Aufnahmetag, Tag 4 der stationären Behandlung und Tag 7 / Entlassungstag eine univariate Analyse mit dem Ziel durchgeführt, mögliche Einflussfaktoren auf die maximale Schmerzintensität zu identifizieren (vgl. Tabelle 14). In einem zweiten Schritt wurden dann signifikante Parameter aus der univariaten Analyse ausgewählt und in die Erstellung einer multivariaten Analyse miteinbezogen. Hierbei zeigten sich für die jeweiligen betrachteten Tage unterschiedliche Ergebnisse: Unabhängige Prädiktoren für die maximale Schmerzintensität am Aufnahmetag waren das Vorhandensein von neuropathischen Schmerzkomponenten (painDETECT Gesamtscore bei Aufnahme) und die körperliche Gesundheit (SF 12). Für den vierten Tag der stationären 
Behandlung war die körperliche Gesundheit (SF 12) der einzige unabhängige Prädiktor für die maximale Schmerzintensität. Als unabhängige Prädiktoren für die maximale Schmerzintensität an Tag 7 / Entlassungstag stellten sich das Vorhandensein von neuropathischen Schmerzkomponenten (painDETECT Gesamtscore bei Aufnahme) und die Somatisierungstendenz (PHQ Gesamtscore bei Aufnahme) heraus (vgl. Tabelle 15).

Tabelle 14 Univariate Analyse: Korrelation zwischen den Parametern und der maximalen Schmerzintensität bei Aufnahme, Tag 4 und Tag 7 / Entlassung

\begin{tabular}{|c|c|c|c|}
\hline Parameter & $\begin{array}{c}\text { Aufnahme } \\
\mathbf{p}\end{array}$ & $\begin{array}{c}\text { Tag } 4 \\
p\end{array}$ & $\begin{array}{c}\text { Tag } 7 \text { / Ent- } \\
\text { lassung } \\
p\end{array}$ \\
\hline Alter & n.s. & n.s. & n.s. \\
\hline BMI & n.s. & n.s. & n.s. \\
\hline Dauer der Zosterschmerzen vor Aufnahme 23 & n.s. & 0,015 & 0,031 \\
\hline PainDETECT Gesamtscore Aufnahme ${ }^{123}$ & $<0,001$ & $<0,001$ & 0,016 \\
\hline SF 12 Körperliche Summenskala ${ }^{123}$ & $<0,001$ & $<0,001$ & $<0,001$ \\
\hline SF 12 Psychische Summenskala & n.s. & n.s. & n.s. \\
\hline DASS Depression Aufnahme & n.s. & n.s. & n.s. \\
\hline DASS Angst Aufnahme & n.s. & n.s. & n.s. \\
\hline DASS Stress Aufnahme & n.s. & n.s. & n.s. \\
\hline DASS Gesamtscore Aufnahme & n.s. & n.s. & n.s. \\
\hline KPI Hilf- und Hoffnungslosigkeit ${ }^{12}$ & 0,012 & 0,040 & n.s. \\
\hline KPI Katastrophisieren & n.s. & n.s. & n.s. \\
\hline KPI Durchhalteappell & n.s. & n.s. & n.s. \\
\hline Tampa Scale of Kinesiophobia Aufnahme 123 & 0,002 & 0,001 & 0,036 \\
\hline PHQ Gesamtscore Aufnahme 123 & 0,012 & 0,041 & 0,003 \\
\hline Druckschmerzschwelle $^{3}$ & n.s. & n.s. & 0,009 \\
\hline
\end{tabular}

Einbeziehung in die multivariate Analyse: ${ }^{1}$ Aufnahmetag ${ }^{2}$ Tag $4{ }^{3}$ Tag 7 / Entlassungstag 
Tabelle 15 Unabhängige Prädiktoren für die maximale Schmerzintensität bei Aufnahme, Tag 4 und Tag 7 / Entlassung

\begin{tabular}{|c|c|c|c|c|}
\hline $\begin{array}{l}\text { Max. } \\
\text { Schmerzin- } \\
\text { tensität }\end{array}$ & $\begin{array}{l}\text { R2 I } \\
\text { korr. } \\
\text { R2 }\end{array}$ & $\mathbf{F}$ & p & Unabhängiger Prädiktor \\
\hline Aufnahmetag & $\begin{array}{l}0,421 \\
0,399\end{array}$ & 18,907 & $<<0,001$ & $\begin{array}{l}\text { PainDETECT Gesamtscore Aufnahme (neuro- } \\
\text { pathische Schmerzkomponente), } \\
\text { Körperliche Gesundheit (SF 12) }\end{array}$ \\
\hline Tag 4 & $\begin{array}{l}0,313 \\
0,298\end{array}$ & 20,069 & $<0,001$ & Körperliche Gesundheit (SF 12) \\
\hline $\begin{array}{l}\text { Tag } 7 \text { / Ent- } \\
\text { lassungstag }\end{array}$ & $\begin{array}{l}0,225 \\
0,190\end{array}$ & 6,380 & 0,004 & $\begin{array}{l}\text { PainDETECT Gesamtscore Aufnahme (neuro- } \\
\text { pathische Schmerzkomponente), Somatisie- } \\
\text { rungstendenz (PHQ Gesamtscore Aufnahme) }\end{array}$ \\
\hline
\end{tabular}

\subsubsection{Einflussfaktoren: Unterschiede zwischen den beiden Gruppen (CP vs. $\mathrm{nCP})$}

Im Folgenden wird erläutert, welche Parameter mit der stärksten kumulativen Intensität der Zosterschmerzen und der stärksten kumulativen bewegungsabhängigen Intensität der Zosterschmerzen in den beiden betrachteten Gruppen (CP, nCP) assoziiert waren. Dafür wurden die folgenden Parameter (ermittelt am Tag der Aufnahme) für die univariate Analyse mit der stärksten bzw. stärksten bewegungsabhängigen kumulativen Intensität der Zosterschmerzen korreliert: Alter, Body mass index (BMI), painDETECT, SF 12-Subskalen, DASS-Subskalen, TSK, PHQ-15, KPI-Kategorien und der Analgetikakonsum (kumulativer MQS).

In der univariaten Analyse zeigte sich, dass bei Patienten ohne vorbestehende chronische Schmerzen der Analgetikakonsum (kumulativer MQS), die Lebensqualität (körperliche Summenskala des SF 12) und das Vorhandensein von neuropathischen Schmerzcharakteristika (painDETECT) mit der stärksten kumulativen Schmerzintensität korrelierten. Bei Patienten mit vorstehenden chronischen Schmerzen korrelierte in der univariaten Analyse ebenfalls die Lebensqualität (körperliche Summenskala des SF 12) und das Vorhandensein von neuropathischen Schmerzcharakteristika (painDETECT) mit der stärksten kumulativen Schmerzintensität. Des Weiteren zeigte sich ein Zusammenhang zwischen dem Vorhandensein von Stress (DASS) und der stärksten kumulativen Schmerzintensität in der Gruppe CP. Weitere Ergebnisse lassen sich Tabelle 16 entnehmen. 
In der multivariaten Analyse stellte sich als unabhängiger Prädiktor sowohl für die stärkste kumulative Intensität der Zosterschmerzen als auch für die stärkste kumulative bewegungsabhängigen Intensität der Zosterschmerzen bei Patienten mit vorbestehenden chronischen Schmerzen die Lebensqualität (körperliche Summenskala des SF 12) und das Vorhandensein von neuropathischen Schmerzcharakteristika (painDETECT) heraus. In der Gruppe der Patienten ohne vorbestehende chronische Schmerzen war der Analgetikakonsum (kumulativer MQS) der einzige unabhängige Prädiktor für die stärkste und stärkste bewegungsabhängige kumulative Intensität der Zosterschmerzen (vgl. Tabelle 17).

Eine Korrelation zwischen Alter und Schmerzintensität konnte in beiden Gruppen nicht ausfindig gemacht werden (CP: kumulative stärkste Schmerzintensität: $p=0,371$, kumulative stärkste bewegungsabhängige Schmerzintensität: $\mathrm{p}=0,466$; $\mathrm{nCP}$ : kumulative stärkste Schmerzintensität $p=0,900$, kumulative stärkste bewegungsabhängige Schmerzintensität $\mathrm{p}=0,693)$.

Tabelle 16 Ergebnisse der univariaten Analyse

\begin{tabular}{|c|c|c|c|c|}
\hline Outcome & Gruppe & Variable & $\begin{array}{l}\text { Kendall- } \\
\text { Tau- b }\end{array}$ & $\mathbf{p}$ \\
\hline $\begin{array}{l}\text { kumulative stärkste } \\
\text { Schmerzintensität } \\
\text { (NRS) }\end{array}$ & $\mathrm{nCP}$ & $\begin{array}{l}\text { Analgetikakonsum (kumulativer } \\
\text { MQS) } \\
\text { Neuropathische Schmerzkompo- } \\
\text { nente (painDETECT) } \\
\text { Körperliche Gesundheit (SF 12) }\end{array}$ & $\begin{array}{r}0,378 \\
0,323 \\
-0,303\end{array}$ & $\begin{array}{l}0,004 \\
0,016 \\
0,022\end{array}$ \\
\hline & $\mathrm{CP}$ & $\begin{array}{l}\text { Neuropathische Schmerzkompo- } \\
\text { nente (painDETECT) } \\
\text { Körperliche Gesundheit (SF 12) } \\
\text { Stress (DASS) }\end{array}$ & $\begin{array}{r}0,323 \\
-0,363 \\
0,374\end{array}$ & $\begin{array}{l}0,028 \\
0,012 \\
0,021\end{array}$ \\
\hline $\begin{array}{l}\text { kumulative stärkste be- } \\
\text { wegungsabhängige } \\
\text { Schmerzintensität } \\
\text { (NRS) }\end{array}$ & $\mathrm{nCP}$ & $\begin{array}{l}\text { Analgetikakonsum (kumulativer } \\
\text { MQS) } \\
\text { Körperliche Gesundheit (SF 12) }\end{array}$ & $\begin{array}{r}0,335 \\
-0,303\end{array}$ & $\begin{array}{l}0,011 \\
0,022\end{array}$ \\
\hline & $\mathrm{CP}$ & $\begin{array}{l}\text { Neuropathische Schmerzkompo- } \\
\text { nente (painDETECT) } \\
\text { Körperliche Gesundheit (SF 12) } \\
\text { Kinesiophobie (TSK) }\end{array}$ & $\begin{array}{r}0,389 \\
-0,366 \\
0,367\end{array}$ & $\begin{array}{l}0,008 \\
0,011 \\
0,014\end{array}$ \\
\hline
\end{tabular}


Tabelle 17 Ergebnisse der multivariaten Analyse

\begin{tabular}{|c|c|c|c|c|c|}
\hline Outcome & Gruppe & $\begin{array}{l}\mathbf{R}^{2} \\
\text { mult. / korr }\end{array}$ & $\mathbf{F}$ & $\mathbf{P}$ & Unabhängige Variable \\
\hline \multirow[t]{2}{*}{$\begin{array}{l}\text { kumulative stärkste } \\
\text { Schmerzintensität }\end{array}$} & $\mathrm{nCP}$ & $\begin{array}{l}0,307 / \\
0,283\end{array}$ & 12,422 & 0,001 & $\begin{array}{l}\text { Analgetikakonsum (kumula- } \\
\text { tiver MQS) }\end{array}$ \\
\hline & $\mathrm{CP}$ & $\begin{array}{l}0,501 / \\
0,453\end{array}$ & 10,542 & $<0,001$ & $\begin{array}{l}\text { Neuropathische Schmerz- } \\
\text { komponente (painDETECT) } \\
\text { Körperliche Gesundheit (SF } \\
\text { 12) }\end{array}$ \\
\hline \multirow[t]{2}{*}{$\begin{array}{l}\text { kumulative stärkste be- } \\
\text { wegungsabhängige } \\
\text { Schmerzintensität }\end{array}$} & $\mathrm{nCP}$ & $0,234 /$ & 8,542 & 0,006 & $\begin{array}{l}\text { Analgetikakonsum (kumula- } \\
\text { tiver MQS) }\end{array}$ \\
\hline & $\mathrm{CP}$ & $\begin{array}{l}0,580 / \\
0,541\end{array}$ & 14,559 & $<0,001$ & $\begin{array}{l}\text { Neuropathische Schmerz- } \\
\text { komponente (painDETECT) } \\
\text { Körperliche Gesundheit (SF } \\
\text { 12) }\end{array}$ \\
\hline
\end{tabular}

\subsection{Ergebnisse aus der Langzeitbefragung}

Die Patienten aus dieser Studie wurden drei (3M) und sechs Monate (6M) nach der Entlassung aus dem Krankenhaus telefonisch kontaktiert und zu Schmerz, Funktionseinschränkungen, Lebensqualität und Analgetikabedarf befragt. Von den 59 in der Studie eingeschlossenen Patienten ließen sich 50 Patienten (85 \%) nach drei Monaten und 49 Patienten (83\%) nach sechs Monaten telefonisch kontaktieren und in die weitere Studie einschließen. Tabelle 18 gibt einen Überblick über das Patientenkollektiv.

Tabelle 18 Charakterisierung des Patientenkollektivs

\begin{tabular}{lcc} 
& 3 Monate & 6 Monate \\
\hline \hline Alter & $70,2 \pm 13,1$ & $71,0 \pm 2,3$ \\
Geschlecht $(\boldsymbol{w} / \mathbf{m})$ & $\mathrm{n}=25(50 \%) / \mathrm{n}=25(50 \%)$ & $\mathrm{n}=24(49 \%) / \mathrm{n}=25(51 \%)$ \\
$\mathrm{nCP} /$ CP & $\mathrm{n}=28(56 \%) / \mathrm{n}=22(44 \%)$ & $\mathrm{n}=27(55 \%) / \mathrm{n}=22(45 \%)$
\end{tabular}

\subsubsection{Prävalenz und Intensität der fortbestehenden Zosterschmerzen}

$35 \%(n=17)$ der Patienten hatten im Zeitraum von drei bis sechs Monaten nach Entlassung aus der stationären Behandlung persistierende Schmerzen im Bereich der von Herpes Zoster 
befallenen Dermatome (NRS $>0$ ). Der Median der maximalen Schmerzintensität dieser Patienten betrug NRS 5 (4-7). Bei $29 \%(n=14)$ aller Patienten konnte unter Berücksichtigung der in dieser Studie gewählten Definition eine Post-Zoster-Neuralgie ausfindig gemacht werden (Schmerzen NRS> 3 im Bereich der von Herpes Zoster befallenen Dermatome, die länger als drei Monate nach der stationären Behandlung fortbestehen). Der Median der maximalen Schmerzintensität bei den Patienten mit einer Post-Zoster-Neuralgie betrug NRS 6 (4-8).

\subsubsection{Vergleich der Patienten mit und ohne einer Post-Zoster-Neuralgie}

Im Folgenden werden die Patienten, die eine Post-Zoster-Neuralgie entwickelt haben (Gruppe PZN), sechs Monate nach der Entlassung hinsichtlich der Stammdaten, Schmerzintensität, Lebensqualität (SF 12), dem Vorhandensein von neuropathischen Schmerzcharakteristika (painDETECT) und dem Analgetikakonsum (MQS) mit den Patienten verglichen, die keine Post-Zoster-Neuralgie entwickelt haben (Gruppe nPZN).

Das Alter der Patienten unterschied sich zwischen den beiden Gruppen nicht. Weibliche Patienten hatten signifikant häufiger eine Post-Zoster-Neuralgie als männliche Patienten (vgl. Tabelle 19).

Tabelle 19 Stammdaten der Patienten mit und ohne einer Post-Zoster-Neuralgie

\begin{tabular}{lccc} 
& PZN & nPZN & Statistik \\
\hline \hline Alter & $73,8 \pm 9,0$ & $69,9 \pm 13,3$ & $t=-0,988, p=0,328$ \\
Geschlecht $(\mathbf{w} / \mathbf{m})$ & $10 / 4$ & $14 / 21$ & $\mathrm{X}^{2}=3,953, \mathrm{p}=0,047$ \\
BMI & $27,4 \pm 4,2$ & $27,5 \pm 4,4$ & $\mathrm{t}=0,085, \mathrm{p}=0,933$
\end{tabular}

Patienten mit einer Post-Zoster-Neuralgie hatten eine signifikant höhere maximale Schmerzintensität (PZN: NRS 6 (4-8), nPZN: 0 (0-1); z=-5,411, p<0,001) und zeigten sechs Monate nach der Entlassung ein signifikant häufigeres Auftreten von neuropathischen Schmerzcharakteristika (painDETECT; PZN: 14,8, nPZN: 2,6; $\mathrm{t}=-6,355, \mathrm{p}<0,001$ ). Der Großteil der Patienten mit einer Post-Zoster-Neuralgie gab sechs Monate nach der Entlassung aus der stationären Behandlung Schmerzen im Bereich des Nervus trigeminus an (vgl. Abbildung 17). 


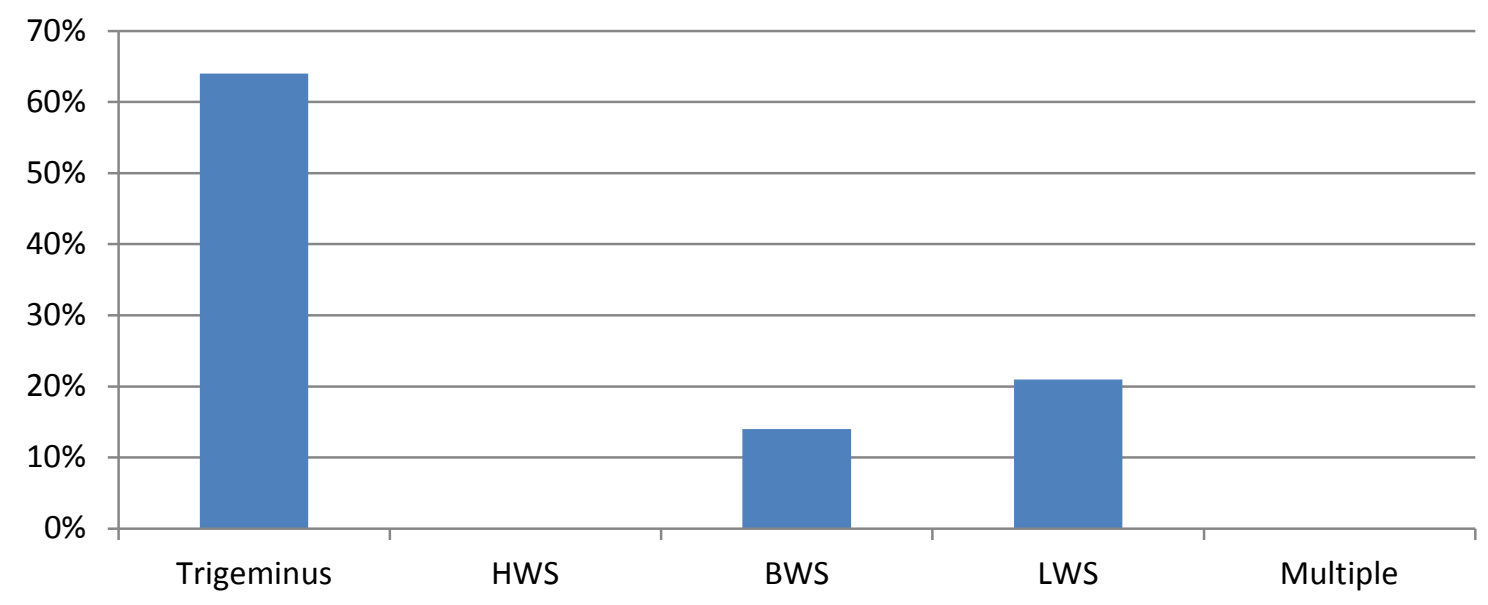

Abbildung 17 Betroffene Dermatome der PZN

$26 \%(n=9)$ der Patienten, die Herpes Zoster im Versorgungsbereich des Nervus trigeminus hatten, entwickelten eine PZN. Von den Patienten, die im Bereich der thorakalen bzw. lumbalen Dermatome einen Befall mit Herpes Zoster zeigten, entwickelten $22 \%(n=2)$ bzw. 50 $\%(n=3)$ eine PZN.

Patienten mit einer Post-Zoster-Neuralgie gaben sechs Monate nach der Entlassung aus dem Krankenhaus eine signifikant geringere Lebensqualität an (vgl. Abbildung 18) und konsumierten häufiger Analgetika (vgl. Tabelle 20).

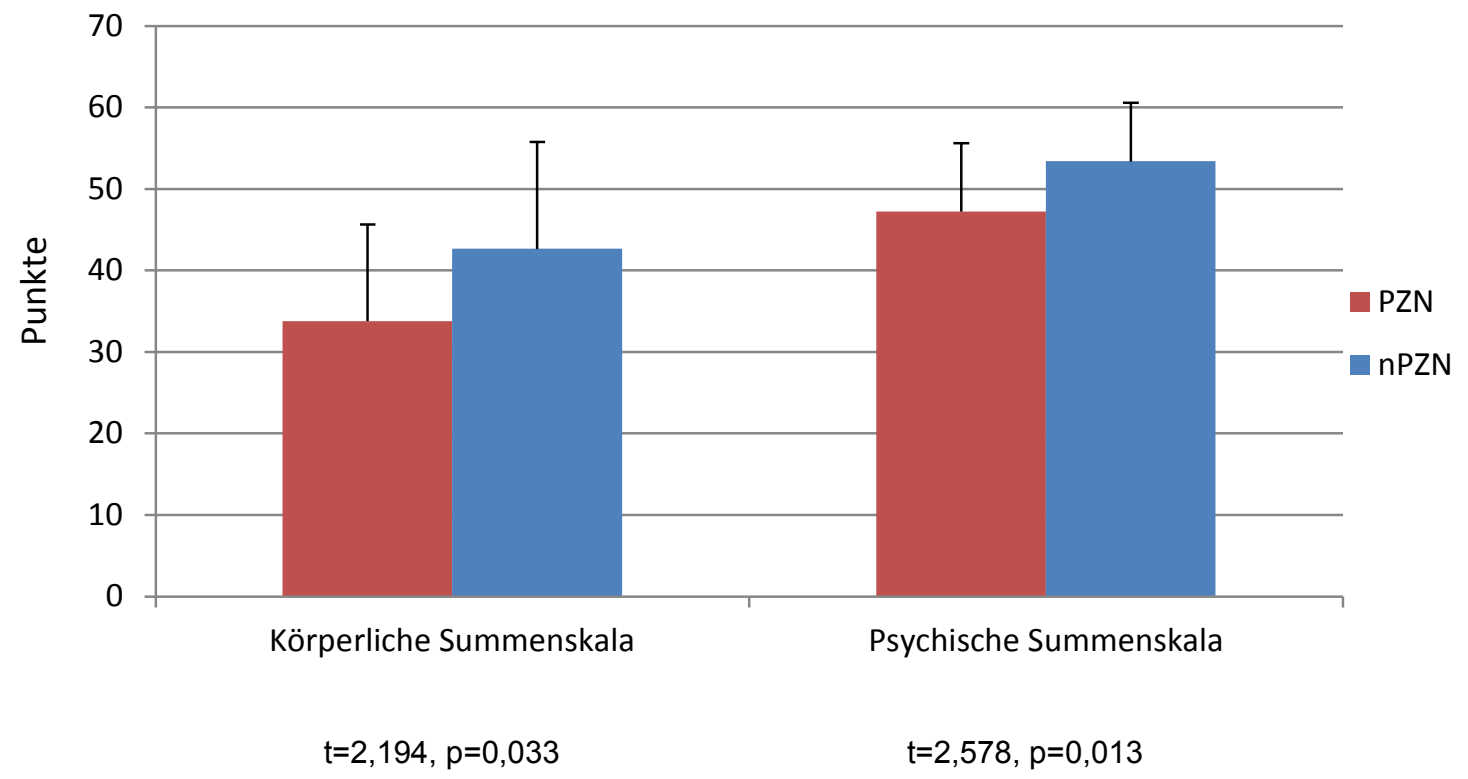

Abbildung 18 Lebensqualität (SF 12), 6 Monate nach der Entlassung 
Tabelle 20 Analgetikakonsum der PZN- und nPZN-Patienten, 6 Monate nach der Entlassung

\begin{tabular}{lcccc} 
& PZN & nPZN & $\mathbf{t}$ & $\mathbf{p}$ \\
\hline \hline Morphinäquivalent & $22,1 \pm 42,4$ & $0,9 \pm 5,1$ & $-2,963$ & 0,005 \\
MQS Opioide & $3,1 \pm 4,1$ & $0,2 \pm 1,1$ & $-3,850$ & $<0,001$ \\
MQS nicht Opioide & $1,8 \pm 3,0$ & $0,9 \pm 3,3$ & $-0,889$ & 0,379 \\
MQS Co-Analgetika & $2,2 \pm 2,7$ & $0,7 \pm 2,1$ & $-2,172$ & 0,035 \\
MQS gesamt & $7,1 \pm 6,2$ & $1,8 \pm 4,0$ & $-3,573$ & $<0,001$
\end{tabular}

\subsubsection{Einfluss vorbestehender chronischer Schmerzen auf die Persistenz von Zosterschmerzen}

Nachfolgend werden die Patienten der Gruppe CP und nCP jeweils drei (3M) und sechs Monate (6M) nach der Entlassung aus dem Krankenhaus hinsichtlich der Stammdaten (vgl. Tabelle 21), der Intensität der persistierenden Zosterschmerzen, der Lebensqualität (SF 12), dem Vorhandensein von neuropathischen Schmerzcharakteristika (painDETECT) und dem Analgetikakonsum (MQS) verglichen.

Tabelle 21 Stammdaten der nCP- und CP-Patienten

\begin{tabular}{lccc|ccc} 
& \multicolumn{3}{c|}{$\mathbf{3}$ Monate } & \multicolumn{3}{c}{$\mathbf{6}$ Monate } \\
& $\mathbf{n C P}$ & $\mathbf{C P}$ & Statistik & $\mathbf{n C P}$ & $\mathbf{C P}$ & Statistik \\
& $\mathbf{n = 2 8}$ & $\mathbf{n = 2 2}$ & & $\mathbf{n = 2 7}$ & $\mathbf{n = 2 2}$ & \\
\hline \hline Alter & $67,0 \pm$ & $74,3 \pm$ & $\mathrm{t}=-2,027$ & $68,4 \pm$ & $74,3 \pm$ & $\mathrm{t}=-1,693$ \\
& 13,2 & 11,9 & $\mathrm{p}=0,048$ & 12,2 & 11,9 & $\mathrm{p}=0,097$ \\
Geschlecht & $15 / 13$ & $10 / 12$ & $\mathrm{X}^{2}=0,325$ & $14 / 13$ & $10 / 12$ & $\mathrm{X}^{2}=0,199$ \\
(w/m) & & & $\mathrm{p}=0,569$ & & & $\mathrm{p}=0,656$ \\
BMI & $28,2 \pm$ & 26,9 & $\mathrm{t}=1,061$ & $28,0 \pm$ & $26,9 \pm$ & $\mathrm{t}=0,9384$ \\
& 4,4 & $\pm 4,4$ & $\mathrm{p}=0,294$ & 4,3 & 4,4 & $\mathrm{p}=0,353$
\end{tabular}

Die mit dem painDETECT erhobene Intensität (NRS) der maximalen Zosterschmerzen unterscheidet sich zwischen den beiden Gruppen nicht signifikant (vgl. Tabelle 22). 
Tabelle 22 Vergleich der Zoster Schmerzintensität zwischen nCP- und CP-Patienten

\begin{tabular}{|c|c|c|c|c|}
\hline & $\mathrm{nCP}$ & $\mathrm{CP}$ & z & p \\
\hline $\begin{array}{l}\text { Max. Zosterschmerzintensität (NRS) im } \\
\text { Zeitraum } 0 \text { bis } 3 \text { Monate nach der Entlas- } \\
\text { sung }\end{array}$ & $2(0-4)$ & $4(1-6)$ & $-1,564$ & 0,118 \\
\hline $\begin{array}{l}\text { Max. Zosterschmerzintensität (NRS) im } \\
\text { Zeitraum } 3 \text { bis } 6 \text { Monate nach der Entlas- } \\
\text { sung }\end{array}$ & $0(0-3)$ & $2(0-4)$ & $-1,327$ & 0,185 \\
\hline
\end{tabular}

Im Screening gab es keinen signifikanten Unterschied hinsichtlich des Vorhandenseins von neuropathischen Schmerzkomponenten (painDETECT; 3M: nCP: 5,9 $\pm 8,7, \mathrm{CP}: 7,9 \pm 6,8$; $\mathrm{t}=$ $-0,895, \mathrm{p}=0,3755$ / 6M: nCP: 5,3 $\pm 7,1, \mathrm{CP}: 7,1 \pm 7,9 ; \mathrm{t}=-0,836, \mathrm{p}=0,407)$. Patienten mit chronischen Schmerzen konsumierten drei und sechs Monate nach der Entlassung häufiger Schmerzmedikamente (MQS; 3M: nCP: 1,4 $\pm 3,8, \mathrm{CP}: 4,6 \pm 6,7 ; \mathrm{t}=-2,182, \mathrm{p}=0,034$ / 6M: nCP $1,1 \pm 2,8, \mathrm{CP}: 6,0 \pm 6,3 ; \mathrm{t}=-3,618, \mathrm{p}<0,001)$. Außerdem zeigten sie eine verminderte gesundheitsbezogene Lebensqualität (Körperliche Summenskala SF 12: 3M: nCP: 44,7 $\pm 11,5$, $\mathrm{CP}: 33,5 \pm 11,0 ; \mathrm{t}=3,461, \mathrm{p}=0,001 / 6 \mathrm{M}: \mathrm{nCP}: 47,6 \pm 10,6, \mathrm{CP}: 30,9 \pm 10,1 ; \mathrm{t}=5,583, \mathrm{p}<0,001$ / Psychische Summenskala SF 12: 3M: nCP: 53,0 $\pm 8,5$, CP: 45,5 $\pm 9,8 ; \mathrm{t}=2,912, \mathrm{p}=0,005$ / 6M: nCP: 54,4 $\pm 6,6, \mathrm{CP}: 48,3 \pm 8,4 ; \mathrm{t}=2,870, \mathrm{p}=0,006)$.

Es bestand kein Zusammenhang zwischen dem Vorhandensein von vorbestehenden chronischen Schmerzen und dem Auftreten einer Post-Zoster-Neuralgie (vgl. Tabelle 23).

Tabelle 23 Verteilung der Patienten mit und ohne vorstehende chronische Schmerzen in den Gruppen PZN und $\mathrm{nPZN}$

\begin{tabular}{|c|c|c|}
\hline & PZN & $\mathrm{nPZN}$ \\
\hline$\overline{C P}$ & $57 \%(n=8)$ & $40 \%(n=14)$ \\
\hline $\mathrm{nCP}$ & $43 \%(n=6)$ & $60 \%(n=21)$ \\
\hline Statistik: & & \\
\hline
\end{tabular}

\subsubsection{Einflussfaktoren auf die Intensität der persistierenden Zosterschmerzen}

Um die Einflussfaktoren auf die Schmerzintensität der persistierenden Zosterschmerzen differenziert darzustellen, wurden eine univariate Analyse (vgl. Tabelle 24) und eine multivariate Analyse (vgl. Tabelle 25) für die abhängige Variable (Outcome Parameter) „maximale 
Zosterschmerzintensität (NRS) im Zeitraum 3 bis 6 Monate nach der Entlassung“ durchgeführt.

Für folgende Parameter zeigte sich eine Assoziation mit der maximalen Intensität (NRS) der Zosterschmerzen im Zeitraum von drei bis sechs Monaten nach der Entlassung aus dem Krankenhaus in der univariaten Analyse: Dauer der stationären Behandlung (Liegetage), Lebensqualität (Körperliche Summenskala des SF 12), Katastrophisieren (KPI), das Vorhandensein einer Kinesiophobie während der stationären Behandlung (TSK) und die Somatisierungstendenz. Außerdem korrelieren die Intensität der Zosterschmerzen (Stärkste Schmerzintensität; QUIPS) an allen ermittelten Tagen (Aufnahme, Tag 4, Entlassung) und die Druckschmerzschwelle mit der maximalen Schmerzintensität.

Unabhängige Prädiktoren für die maximalen Intensität (NRS) der Zosterschmerzen im Zeitraum von drei bis sechs Monaten nach der Entlassung aus dem Krankenhaus waren in der multivariaten Analyse die Dauer der stationären Behandlung (Liegetage) und die stärkste Intensität der Zosterschmerzen am Entlassungstag (QUIPS). 
Tabelle 24 Ergebnisse der univariate Analyse

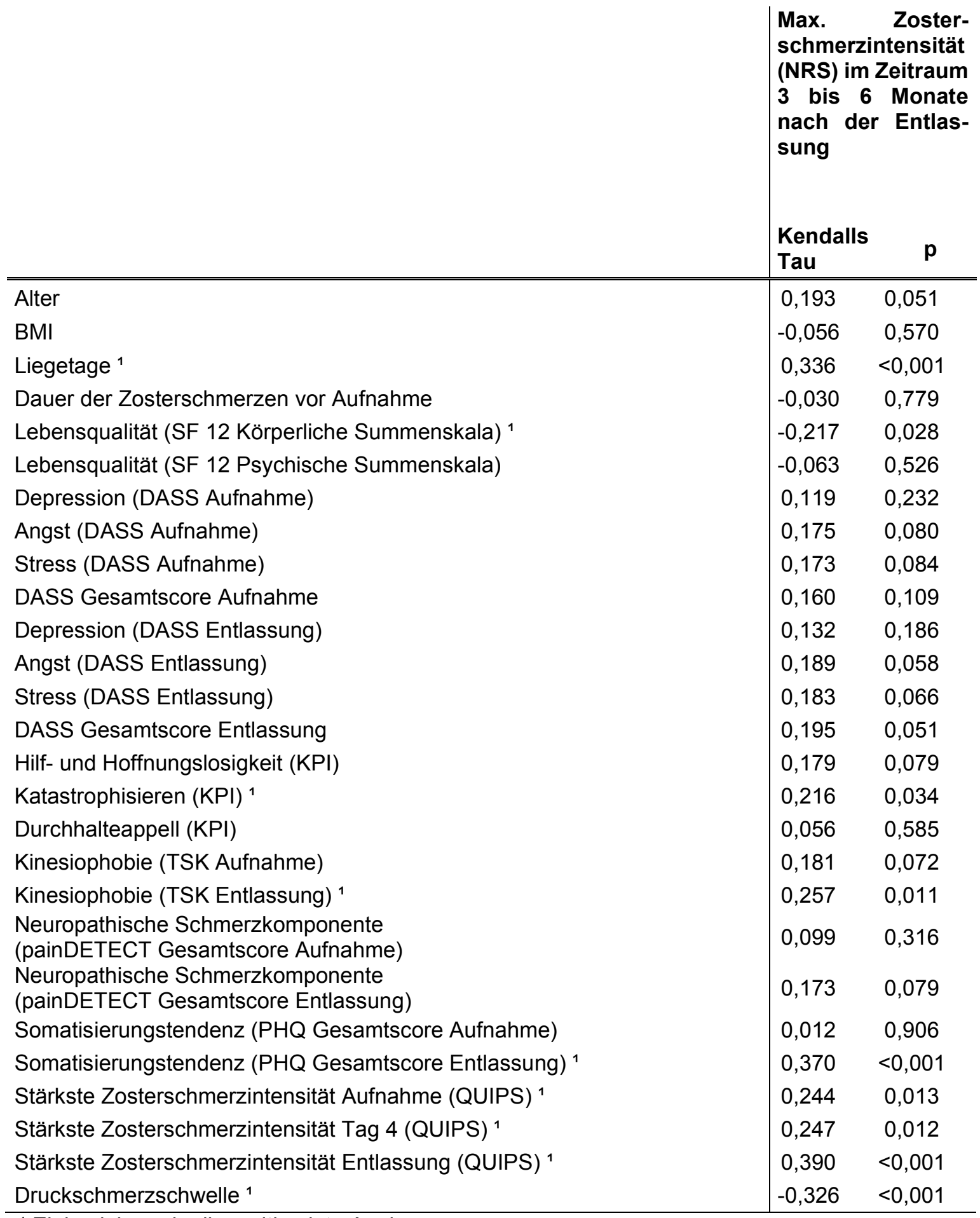

${ }^{1}$ Einbeziehung in die multivariate Analyse 
Tabelle 25 Ergebnisse der multivariaten Analyse

\begin{tabular}{lccc|l} 
& $\begin{array}{c}\text { R2 I } \\
\text { korr. R2 }\end{array}$ & F & p & Unabhängiger Prädiktor \\
\hline $\begin{array}{l}\text { Max. Zosterschmerzintensität (NRS) } \\
\text { im Zeitraum 3 bis 6 Monate nach der }\end{array}$ & 0,338 & 10,966 & $<0,001$ & Liegetage \\
Entlassung & 0,307 & & $\begin{array}{l}\text { Stärkste Zoster- } \\
\text { schmerzintensität Entlas- } \\
\text { sung (QUIPS) }\end{array}$
\end{tabular}




\section{Diskussion}

\subsection{Patienten mit vorbestehenden chronischen Schmerzen}

Aus dem operativen Bereich ist bekannt, dass chronische Schmerzen ein relevanter und konsistenter Risikofaktor für eine hohe Intensität akuter postoperativer Schmerzen sind. Sie gehen nach Operation auch mit einer verlangsamten Rehabilitation und Funktion einher (Gerbershagen et al. 2014; Gerbershagen et al. 2009; Hinrichs-Rocker et al. 2009; Ip et al. 2009; Erlenwein et al. 2016). Die vorgelegte Untersuchung konnte darstellen, dass auch bei Patienten mit akuter Herpes-Zoster-Infektion vorbestehende chronische Schmerzen mit einer höheren Schmerzintensität der Zosterschmerzen assoziiert sind. Außerdem zeigten Patienten mit vorbestehenden chronischen Schmerzen relevante funktionelle Einschränkungen und erhöhten psychologischen Distress. Diese Erkenntnisse sind insbesondere wichtig, weil viele Menschen unter chronischen Schmerzen leiden. Dabei wird in der Literatur eine Prävalenz zwischen 19 und 28,3 \% angegeben, im Patientenkollektiv der vorliegenden Untersuchung betrug die Prävalenz in einem allerdings älteren Patientenkollektiv sogar $42 \%$ (Kurita et al. 2012; Breivik et al. 2006; Häuser et al. 2015).

\subsubsection{Einflussfaktoren auf die Intensität der akuten Zosterschmerzen}

In der vorliegenden Untersuchung ist bei den Patienten mit vorbestehenden chronischen Schmerzen die Intensität der Zosterschmerzen erstens assoziiert mit der gesundheitsbezogenen Lebensqualität und zweitens mit dem Vorhandensein von neuropathischen Schmerzcharakteristika.

Das Vorhandensein von neuropathischen Schmerzcharakteristika scheint bei Patienten mit vorbestehenden chronischen Schmerzen neben nozizeptiven Mechanismen ein wesentlicher Grund für die Generierung stärkerer akuter Zosterschmerzen zu sein. Diese Abhängigkeit zeigte sich zwar auch bei der Betrachtung des gesamten Patientenkollektives. Wurden in die Analyse aber nur Patienten ohne chronische Schmerzen einbezogen, so ließ sich dieser $\mathrm{Zu}-$ sammenhang nicht darstellen. Ursächlich hierfür könnte eine zentrale Sensibilisierung sein, welche bei Patienten mit chronischen Schmerzen beschrieben worden ist (Koroschetz et al. 2011; Rehm et al. 2010). Bei der zentralen Sensibilisierung kommt es durch langanhaltende Schmerzen zu funktionellen Veränderungen im zentralen Nervensystem, die zu einer erhöhten Erregbarkeit von Neuronen führen. Bereits geringste Verletzungen von Gewebe können 
so zu ausgeprägten Schmerzen führen und sind somit Ausdruck einer erhöhten Schmerzempfindlichkeit (Oosterwijck et al. 2013). In experimentellen Szenarien konnte diese Beziehung unter anderem anhand einer verminderten Druckschmerzschwelle bei Patienten mit chronischen Schmerzen gezeigt werden (Petzke et al. 2003; Giesecke et al. 2004). Eine erhöhte Druckschmerzempfindlichkeit der chronischen Schmerzpatienten konnte auch in der vorliegenden Studie reproduziert werden. Hingegen ließ sich eine Beziehung zwischen Druckschmerzempfindlichkeit und akuter Schmerzintensität, wie sie in klinischen Untersuchungen aus dem operativen Bereich ausfindig gemacht werden konnte, für das in dieser Studie untersuchte Patientenkollektiv nicht darstellen (Pan et al. 2006; Hsu et al. 2005). Dies liegt möglicherweise daran, dass die Erfassung der Druckschmerzschwelle nicht wie in den operativen Untersuchungen präoperativ, also in einem „,neutralen“ Intervall erfolgte, sondern erst nach Beginn der Erkrankung und der Beschwerden.

Wie bereits in der Literatur beschrieben, war auch in dieser Untersuchung die gesundheitsbezogene Lebensqualität sowohl für die körperliche als auch für die psychische Gesundheit bei den Patienten mit chronischen Schmerzen vermindert (Løyland et al. 2010; Antonopoulou et al. 2009; Lee et al. 2005). Außerdem konnte für die Patienten mit vorbestehenden chronischen Schmerzen ein Zusammengang zwischen einer verminderten gesundheitsbezogenen Lebensqualität und der Intensität von akuten Zosterschmerzen gezeigt werden. Dieser Zusammenhang ist nach Wissen des Autors bisher in der Literatur nicht beschrieben worden.

Wie auch in der allgemeinen Bevölkerung nahm der Anteil an Patienten mit chronischen Schmerzen mit zunehmenden Alter im hier untersuchten Studienkollektiv zu, sodass das Alter eine mögliche Erklärung für die höhere Schmerzintensität sein könnte (Dworkin et al. 2001). Wenn auch ein signifikanter Unterschied in der Intensität der Zosterschmerzen zwischen den untersuchten Gruppen vorlag und in der Gruppe mit vorbestehenden chronischen Schmerzen der Altersdurchschnitt signifikant höher war, zeigte sich in der Analyse der einzelnen Einflussfaktoren kein Zusammenhang zwischen Alter und Intensität der akuten Zosterschmerzen. Dies zeigte sich sowohl in der gruppenspezifischen Einflussanalyse als auch für das Gesamtkollektiv. 


\subsubsection{Funktionalität}

Patienten mit chronischen Schmerzen hatten häufiger funktionelle Einschränkungen. Dies stellte sich vor allem in Bezug auf Einschränkungen durch Schmerz bei Mobilisation, der Schlafqualität und der Stimmung dar. Entsprechende Einschränkungen sind jedoch bereits grundsätzlich im Kontext chronischer Schmerzen häufig beschrieben, sodass hier zu einem relevanten Anteil der chronische Schmerz als Komorbidität zur Geltung kommt (Taylor et al. 2005; Karaman et al. 2014; Tang et al. 2007). Das Ausmaß der funktionellen Einschränkungen mag Ausdruck einer höheren Belastung durch die akute Erkrankung bei geringerer Stresstoleranz und maladaptiven krankheitsbezogenen Verarbeitungsmechanismen sein. Jene bestehen ebenfalls häufig im Kontext chronischer Schmerzen (Hildebrandt et al. 1992). Ferner zeigte sich, dass diese Patientengruppe im Trend häufiger Nebenwirkungen und Zusatzsymptome wie Müdigkeit und Übelkeit angab.

\subsubsection{Psychologischer Distress}

Von Untersuchungen aus dem operativen Bereich ist bekannt, dass die postoperative Schmerzintensität unter anderem von psychologischen Charakteristika wie Depression, Angst und Stress, beeinflusst wird (Ip et al. 2009; Kroenke et al. 2002; Noiseux et al. 2014). Das Auftreten von Depressionen ist für Patienten mit chronischen Schmerzen mehrfach beschrieben worden (Holmes et al. 2013; Arnow et al. 2006). Im Gegensatz hierzu zeigte sich für das hier untersuchte Kollektiv von Patienten mit akuter Zosterinfektion bei der Ersterfassung psychologischen Distresses (Depression, Angst und Stress) kein signifikanter Unterschied zwischen den Patienten mit und ohne vorbestehende chronische Schmerzen. Ein Faktor für die abweichenden Ergebnisse ist möglicherweise, dass das Patientenkollektiv in dieser Studie vorselektioniert ist. Hier wurden nur Patienten betrachtet, die aufgrund der Schwere der akuten Zoster Erkrankung stationär behandelt werden mussten. Daher ist es denkbar, dass ein nicht zu unterschätzender Einfluss durch den Zeitpunkt der Befragung bedingt ist: Die Erhebung der Distress-Faktoren erfolgte in beiden Gruppen erst nach der stationären Aufnahme in der akuten Erkrankungsphase. Somit könnten die Zosterschmerzen sowohl bei den Patienten mit als auch ohne vorbestehende chronische Schmerzen gleichermaßen Symptome einer Depression hervorgerufen haben (Barman 2015).

Auch zeigten die Patienten mit vorbestehenden chronischen Schmerzen eine höhere Tendenz zur Somatisierung und hatten ein häufigeres Auftreten von „Hilf- und Hoffnungslosigkeit““ als Schmerzverarbeitungsmuster. Somatisierung stellt bei Patienten mit chronischen 
Schmerzen eine häufige Komorbidität dar (Birket-Smith 2001). Außerdem konnten Studien zeigen, dass negative Emotionen wie Hilf- und Hoffnungslosigkeit ebenso wie Angststörungen mit chronischen Schmerzen assoziiert sind (Kiosses et al. 2017; McWilliams et al. 2003). Des Weiteren stellten sich bei den Patienten mit zusätzlich zum akuten Herpes Zoster bestehenden chronischen Schmerzen häufiger bewegungsbezogene Ängste dar (Nicholas et al. 2009). Bewegungsbezogene Angst ist als wichtiger Chronifizierungsfaktor bei muskuloskelettalen Schmerzen bekannt (Houben et al. 2005).

\subsection{Risikoindikatoren für ungünstige Krankheitsverläufe}

Ein Ziel dieser Studie war es, Risikoindikatoren ausfindig zu machen, die mit ungünstigen Krankheitsverläufen unabhängig von vorbestehenden chronischen Schmerzen einhergehen. Dabei wurde insbesondere der Frage nachgegangen, welche Indikatoren mit einer hohen Schmerzintensität der akuten Zosterschmerzen beziehungsweise mit einer hohen Schmerzintensität der persistierenden Zosterschmerzen assoziiert sind.

Dabei stellte sich heraus, dass die Indikatoren, die mit einer hohen Intensität der akuten Zosterschmerzen einhergehen, sich im Verlauf der stationären Behandlung der Patienten unterscheiden. Für den Beginn des Krankheitsverlaufs erscheint das Vorkommen von neuropathischen Schmerzkomponenten und eine verminderte körperliche Gesundheit (SF 12) für die Generierung einer hohen Intensität der Zosterschmerzen mit verantwortlich zu sein. Das Schmerzbild einer akuten Zosterinfektion ist durch nozizeptive und neuropathische Schmerzen charakterisiert (Dworkin et al. 2008; Johnson 2007; Oaklander 2008). Es ist denkbar, dass die Patienten unzureichend analgetisch therapiert wurden, denn neuropathische Schmerzen sind komplex und die Behandlung stellt eine Herausforderung dar (Dworkin et al. 2010). Dieses wird deutlich anhand einer Studie, in der Patienten mit neuropathischen Schmerzen in nicht einmal der Hälfte der Fälle eine klinisch bedeutsame Schmerzlinderung erfahren haben (O’Connor 2009). Auch ist möglich, dass antineuropathische Medikamente noch keine ausreichende Wirkung erzielt hatten, da hier bis zum Wirkeintritt Tage bis Wochen notwendig sind (Binder und Baron 2016).

Zum Ende der stationären Behandlung (Tag 7 / Entlassung) konnte ebenfalls das Vorkommen von neuropathischen Schmerzkomponenten als Indikator für eine hohe Schmerzintensität identifiziert werden. Außerdem zeigte sich, dass die Somatisierungstendenz (PHQ) mit der Generierung einer hohen Intensität der Zosterschmerzen assoziiert zu sein scheint. Es ist bekannt, dass psychologische Faktoren wie Somatisierungsstörungen ein Risikofaktor für 
akute und anhaltende Schmerzen nach einer Operation sind (Pogatzki-Zahn et al. 2009). Für Patienten, die eine Kniegelenksprothese erhielten, konnte gezeigt werden, dass eine Somatisierungsstörung mit höheren postoperativen Schmerzen einherging (Bierke et al. 2016).

Folgende Indikatoren waren sechs Monate nach der Entlassung der Patienten mit der maximalen Intensität der persistierenden Zosterschmerzen assoziiert: 1. Dauer der stationären Behandlung. 2. Die maximale Intensität der Zosterschmerzen am Entlassungstag. Bisher wurden in mehreren Studien Risikofaktoren für das Auftreten einer Post-Zoster-Neuralgie identifiziert (vgl. Kapitel 1.3.2). Welche Faktoren Einfluss auf die Intensität der persistierenden Zosterschmerzen haben, wurde nach Wissen des Autors bisher nicht untersucht.

Eine im Vergleich verlängerte stationäre Liegezeit könnte Ausdruck einer schweren HerpesZoster-Infektion beziehungsweise der Notwendigkeit einer komplexen Schmerztherapie sein und daher mit der maximalen Intensität der persistierenden Zosterschmerzen in Verbindung stehen.

Dass die Intensität der Zosterschmerzen am Entlassungstag Einfluss auf die Intensität der persistierenden Zosterschmerzen hat, geht einher mit Studien aus dem chirurgischen Bereich, in welchen das Ausmaß der Schmerzen nach einer Operation als etablierter Prädiktor für das Auftreten von chronischen Schmerzen identifiziert worden ist (Schnabel und Pogatzki-Zahn 2010; Althaus et al. 2012). Analog hierzu konnte gezeigt werden, dass ein starker akuter Zosterschmerz ein Risikofaktor für das Auftreten einer Post-Zoster-Neuralgie ist (Jung et al. 2004; Scott et al. 2003; Whitley et al. 1998; Dworkin et al. 2001).

Des Weiteren entwickelten Frauen in der vorliegenden Studie häufiger eine Post-ZosterNeuralgie als Männer. Weibliches Geschlecht wird in viele Studien als ein Risikofaktor für das Entwickeln einer Post-Zoster-Neuralgie gesehen (Jung et al. 2004; Scott et al. 2003; Whitley et al. 1998; Dworkin et al. 2001). Die Ergebnisse eines umfangreichen Review kommen aber zu dem Ergebnis, dass weibliches Geschlecht kein eindeutiger Risikofaktor zu sein scheint (Forbes et al. 2016a).

Patienten, die eine Post-Zoster-Neuralgie entwickelt haben, hatten eine geringere subjektive gesundheitsbezogene Lebensqualität. Studien zum Einfluss der Post-Zoster-Neuralgie auf die Lebensqualität untermauern diese Ergebnisse (Johnson et al. 2010; Drolet et al. 2010; Schmader et al. 2007). Des Weiteren hatten Patienten mit einer Post-Zoster-Neuralgie erwartungsgemäß eine höhere maximale Schmerzintensität und konsumierten höhere Mengen 
an Analgetika. Insbesondere erhielten diese Patienten häufiger Opioide und Co-Analgetika. Dieses entspricht der empfohlenen Therapie bei neuropathischen Schmerzen bzw. einer Post-Zoster-Neuralgie (Edelsberg et al. 2011; Goßrau 2014). Außerdem zeigten sie bei Aufnahme analog zu weiteren Studien ein häufigeres Auftreten von neuropathischen Schmerzcharakteristika (Gilden et al. 2000; Baron 2006).

Die hohe Prävalenz der Post-Zoster-Neuralgie in dieser Studie und die damit verbundenen Einschränkungen der Patienten in Hinblick auf die Lebensqualität verdeutlichen die Wichtigkeit der Prävention und Therapie der Post-Zoster-Neuralgie, reflektieren aber auch die Rekrutierung einer Hochrisikogruppe mit der Notwendigkeit einer stationären Behandlung.

\subsection{Analgetikakonsum}

Eine konsequente Schmerztherapie spielt in der Behandlung des Herpes Zoster eine entscheidende Rolle (Gross et al. 2003). Daher wurde der Analgetikagebrauch sowohl bei den Patienten mit als auch ohne vorbestehende chronische Schmerzen im Rahmen der vorliegenden Untersuchung ausführlich betrachtet. Hier konnten keine signifikanten Unterschiede in der Menge der konsumierten Analgetika zwischen den beiden Gruppen ausfindig gemacht werden. Dieses Ergebnis ist besonders hervorzuheben, denn aufgrund der höheren Intensität der Zosterschmerzen bei den Patienten mit vorbestehenden chronischen Schmerzen hätte man in dieser Gruppe auch einen höheren Analgetikakonsum vermutet.

Allerdings erhielten diese Patienten höhere Dosen von Opioiden, wenn die Äquivalenzdosis in mg Morphin betrachtet wurde. Dieses ist aber darauf zurückzuführen, dass diese Patienten schon vor der stationären Behandlung, vermutlich aufgrund ihrer chronischen Schmerzen, Opioide eingenommen haben und diese während des stationären Aufenthaltes weiter verabreicht wurden. Betrachtet man nur die Opioide, die zusätzlich aufgrund der Zosterschmerzen verabreicht wurden, lässt sich auch hier kein signifikanter Unterschied zwischen den beiden Gruppen feststellen. Hervorzuheben ist, dass bei den Patienten ohne vorbestehende chronische Schmerzen die Intensität der Zosterschmerzen nur mit der Menge der konsumierten Analgetika assoziiert war. In der Gruppe der Patienten mit vorbestehenden chronischen Schmerzen konnte dieser Zusammenhang entgegen der Erwartung nicht gezeigt werden. Dieses bedeutet, dass mit der Zunahme der Schmerzintensität die Menge an konsumierten Analgetika nicht zugenommen hat und könnte daher ebenfalls auf eine Unterversorgung der Patienten mit vorbestehenden chronischen Schmerzen hinweisen. Dies wird dadurch unter- 
mauert, dass diese Patienten ein vermehrtes Verlangen nach Analgetika hatten und insgesamt weniger zufrieden mit der Schmerztherapie waren. Andererseits machen diese Daten auch die Herausforderung einer angemessenen Behandlung neuer und akuter Schmerzen bei Patienten mit bereits vorbestehenden Schmerzen deutlich, da von diesen Patienten auch im Trend mehr Begleitsymptome berichtet wurden.

\subsection{Versorgungsaspekte}

Wie bereits deutlich geworden ist, lassen die Ergebnisse dieser Untersuchung eine Unterversorgung, bzw. eine schwierigere und komplexere Therapie mit Analgetika bei Patienten mit chronischen Schmerzen vermuten. Dieses geht einher mit einer Studie von Maier et al. 2010, die zeigt, dass die Versorgung der Schmerzen in den konservativen Fächern schlechter ist als in operativen Fächern. Neben dem Leid, welches den Patienten mit chronischen Schmerzen widerfährt, rücken auch zunehmend finanzielle Aspekte in den Vordergrund. In dieser Studie war die Liegedauer der Patienten mit vorbestehenden chronischen Schmerzen signifikant erhöht. Aus dem operativen Bereich ist bekannt, dass durch ungenügend behandelte starke Schmerzen der stationäre Aufenthalt der Patienten verlängert wurde. Das Einhalten der in den DRGs vorgesehen Liegezeiten wird sowohl durch die Unterbehandlung als auch durch die schwierigere Einstellung der Schmerzen bei komplexen Patienten erschwert (Kehlet 2004; Capdevila et al. 1999).

Vor dem Hintergrund einer ethisch-moralischen Verpflichtung einer angemessenen Schmerztherapie und der ökonomischen Bedeutung ungenügend behandelter Schmerzen erscheinen die Einführung von festen Schmerzkonzepten und die Einbeziehung von Akutschmerzdiensten in konservativen Bereichen sinnvoll. Korczak et al. 2013 konnten zeigen, dass es durch die Etablierung von Akutschmerzdiensten zu einer Kostenreduktion kommt, die im Wesentlichen auf einer Verkürzung der Krankenhausverweildauer und einer verringerten Wiederaufnahmerate der Patienten beruht. Doch gerade bei der Etablierung von festen Schmerzkonzepten beziehungsweise Akutschmerzdiensten besteht ein deutlicher Optimierungsbedarf. Eine Studie aus dem nicht-operativen Bereich kommt zu dem Ergebnis, dass in nur der Hälfte der deutschen Krankenhäuser schriftliche Standards zur Akutschmerztherapie existieren (Erlenwein et al. 2014). Außerdem verfügen nur $58 \%$ der deutschen Krankenhäuser über eine ausgewiesene schmerzmedizinische Versorgung (Erlenwein et al. 2017). 
Bei der Einführung beziehungsweise Optimierung von festen Schmerzkonzepten ist es wichtig, Patienten mit vorbestehenden chronischen Schmerzen besondere Aufmerksamkeit zu schenken. Denn die vorliegende Untersuchung lässt vermuten, dass diese Patienten unzureichend analgetisch therapiert wurden. Außerdem waren chronische Schmerzen ein Risikoindikator für die Entwicklung von starken Akutschmerzen. Auch in Anbetracht der hohen Prävalenz hat die Identifizierung von Patienten mit chronischen Schmerzen daher eine entscheidende Bedeutung für eine adäquate Schmerztherapie.

Diese Studie stellt ferner dar, dass es unzureichend ist, Schmerzen ausschließlich eindimensional anhand von Schmerzskalen in Bezug zur Schmerzintensität zu erheben. Aufgrund der Einschränkungen in den Funktionen und des psychologischen Disstress der Patienten während der stationären Behandlung sollten diese Faktoren in die Bewertung der Schmerzen mit einbezogen werden. Bei Bedarf sollte dann ein multimodales Therapieregime zur Anwendung kommen (Casser und Nagel 2016) . Eine regelmäßige Reevaluation der Schmerzen ist notwendig, um den Therapieerfolg zu kontrollieren und die Analgesie zu adaptieren. Wie diese Studie darstellt, kommt auch der Identifizierung neuropathischer Schmerzcharakteristika eine besondere Bedeutung zu, da diese für die Generierung eines starken Akutschmerzes mit verantwortlich sind. Die Behandlung von neuropathischen Schmerzen sollte auch in die genannten Standards einfließen. Dabei gilt es zu beachten, dass die Therapie von neuropathischen Schmerzen sehr komplex ist und anhand der Leitlinien erfolgen sollte (Dworkin et al. 2010; Werner et al. 2017a).

\subsection{Definition der chronischen Schmerzen und der Post-Zoster-Neuralgie}

In dieser Studie wurden chronische Schmerzen definiert als Schmerzen, die länger als sechs Monate bestehen (Gerbershagen et al. 2009). Unter Berücksichtigung dieser Definition ist die Prävalenz von Patienten mit chronischen Schmerzen in der vorliegenden Untersuchung mit $42 \%$ wesentlich höher als in der Literatur angegeben. Die Prävalenz von chronischen Schmerzen in der Bevölkerung ist Gegenstand zahlreicher Untersuchungen und variiert von Studie zu Studie. Differenzen ergeben sich hier vor allen in der Betrachtung unterschiedlicher Patientenkollektive (vgl. Tabelle 26). 
Tabelle 26 Prävalenz chronischer Schmerzen

\begin{tabular}{ll|l} 
Prävalenz & Patientenkollektiv & Studie \\
\hline \hline $26,8 \%$ & $>16$ Jahre, inklusive Krebspatienten & Kurita et al. 2012 \\
\hline $19 \%$ & $>18$ Jahre, exklusive Krebspatienten & Breivik et al. 2006 \\
\hline $28,3 \%$ & $>14$ Jahre, inklusive Krebspatienten, & Häuser et al. 2015 \\
& Schmerzen länger als drei Monate & \\
&
\end{tabular}

Ursächlich für die hohe Prävalenz in dieser Studie könnte zum einen die Selektion der Patienten durch die stationäre Aufnahme und zum anderen das hohe Alter (68 \pm 15 Jahre) der betrachteten Patienten sein. Denn es ist bekannt, das mit zunehmenden Alter die Prävalenz von chronischen Schmerzen zunimmt (Häuser et al. 2015).

Bei der näheren Differenzierung der chronischen Schmerzerkrankung zeigte sich, dass mehr als die Hälfte (56 \%) der Patienten mit vorbestehenden chronischen Schmerzen hierdurch beeinträchtigt war, welches einem Chronic Pain Grade Stadium III und IV entspricht. Häuser et al. 2015 hatten Daten von über 2500 Personen aus einer für die deutsche Bevölkerung repräsentativen Stichprobe ausgewertet und ordneten 7,3 \% der betrachteten Personen dem CPG Stadium III und IV zu. Für das Vereinte Königreich (UK) konnte in einem Review gezeigt werden, dass die Prävalenz von Patienten mit beeinträchtigenden chronischen Schmerzen (CPG Stadium III und IV) zwischen 10,4 \% und 14,3 \% liegt (Fayaz et al. 2016). In dieser Untersuchung gaben $29 \%$ der Patienten drei Monate nach der Entlassung Schmerzen NRS $>3$ im Bereich des vom Herpes Zoster befallenden Dermatoms an (Definition der Post-Zoster-Neuralgie in dieser Studie). Die in der Literatur beschriebene Prävalenz der Post-Zoster-Neuralgie variiert je nach Definition: Schmerzintensität (NRS $>0$ oder NRS $>3$ ) nach Abheilung einer Zosterinfektion innerhalb von 4 Monaten / 90 Tagen / 6 Wochen (vgl. Kapitel 1.3.2). Außerdem schließen Studien immunsupprimierte Patienten in das Patientenkollektiv ein (Forbes et al. 2016b) und häufig werden nur Patienten aus dem ambulanten Bereich betrachtet. In einer weiteren Studie wurden nur Patienten über 50 Jahre in die Studie eingeschlossen (Benbernou et al. 2011) und nicht alle Patienten erhielten beispielsweise eine antivirale Therapie (Bouhassira et al. 2012). Die Prävalenz der Post-Zoster-Neuralgie aus 
den eben genannten Studien variiert daher von 5,8 \% bis $33 \%$ aller Patienten mit einer vorrausgegangenen Herpes-Zoster-Infektion. Dieses zeigt, dass die Prävalenz der Post-ZosterNeuralgie stark abhängig ist von der Definition.

\subsection{Stärken und Schwächen}

Nach Wissen des Autors konnte in der vorliegenden Untersuchung erstmals gezeigt werden, dass bei Patienten mit akuter Herpes-Zoster-Infektion vorbestehende chronische Schmerzen mit einer höheren Intensität der Zosterschmerzen einhergehen.

Für diese Studie wurden Patienten mit Herpes Zoster ausgewählt, da dieses Krankheitsbild mit einem gut abzugrenzenden nicht-operativen Akutschmerz vergesellschaftet ist. Außerdem können diese Schmerzen nicht selten chronifizieren und als Post-Zoster-Neuralgie fortbestehen (Benbernou et al. 2011). Die Auswahl von Patienten mit Herpes Zoster ist daher als Beispiel für konservative Schmerzen sehr gut geeignet.

Eine weitere Stärke der vorliegenden Studie ist die gleichzeitige Berücksichtigung klinischer, psychologischer und physiologischer Parameter. Dieses ist wichtig, da es sich bei Schmerzen um ein multidimensionales Konstrukt handelt, welches eine multidimensionale Erfassung erfordert (Dworkin et al. 2016). Daher erfolgte zu Beginn der stationären Behandlung eine sehr differenzierte Charakterisierung des Patientenkollektivs. Neben der Schmerzintensität wurden auch funktionelle und psychologische Outcome-Parameter zur Beurteilung des klinischen Verlaufs erfasst. Auch die chronischen Schmerzen wurden in dieser Studie sehr genau differenziert. Eine weitere Stärke der vorliegenden Untersuchung liegt in der kontinuierlichen Beobachtung von Beginn der stationären Behandlung bis zum Entlassungstag und der nachfolgenden Befragung der Patienten drei und sechs Monate nach der Entlassung.

Da die Therapie der von Zoster ausgehenden Schmerzen sehr komplex ist (Allegri et al. 2014; Marchettini et al. 2006; Johnson 2007) wurde im Vergleich zu Studien aus dem postoperativen Bereich nicht nur der Opioidverbrauch berücksichtigt, sondern zusätzlich auch NichtOpioide, Antidepressiva und Antikonvulsiva. Um dennoch eine Vergleichbarkeit der Patienten hinsichtlich des Analgetikakonsums zu erreichen, wurde dieser durch den MQS quantifiziert, der allerdings nur eine grobe Vergleichbarkeit des Analgetikakonsums ermöglicht, weshalb diese Daten mit Vorsicht zu betrachten sind. 
Die Verteilung der Geschlechter und des BMI war in beiden Gruppen homogen. Durch den großen Anteil von Patienten mit chronischen Schmerzen lassen sich die beiden Gruppen gut vergleichen. Allerdings waren die betrachteten Gruppen bezogen auf das Alter inhomogen. An dieser Stellte könnte argumentiert werden, dass nicht etwa das Vorhandensein von chronischen Schmerzen mit einer höheren Intensität der Zosterschmerzen einhergeht, sondern das Alter der Patienten (Dworkin et al. 2001). Dies ist aber unwahrscheinlich, da das Alter in der univariaten Analyse nicht mit der Intensität der Zosterschmerzen korrelierte.

Zwar wurden alle Patienten speziell nach Schmerzen durch die Herpes-Zoster-Infektion befragt, eine Vermischung der Zosterschmerzen und der chronischen Schmerzen in der subjektiven Bewertung der Patienten lässt sich aber nicht gänzlich ausschließen. Es kann daher auch nicht eindeutig ausgeschlossen werden, dass die höhere Schmerzintensität der Patienten mit vorbestehenden chronischen Schmerzen einen größeren Leidensdruck widerspiegelt und eine schlechte Abgrenzung (trotz unterschiedlichem Fragemodus) durch den Patienten erfolgte. Die genutzten Instrumente zur Beurteilung der Funktionen und Beeinträchtigung im akuten Verlauf (QUIPS) sind bisher nur für die postoperative Nutzung validiert und bisher nicht an einem nicht-operativen Patientenkollektiv wissenschaftlich genutzt worden.

Die Patienten waren aufgrund des Studiendesigns sehr vorselektioniert. Daher lassen sich die Ergebnisse dieser Studie nur eingeschränkt auf ambulante Patienten mit Herpes Zoster übertragen. Dieses wird beispielsweise dadurch verdeutlicht, dass bei $68 \%$ der Patienten das Dermatom des Nervus Trigeminus mit einem oder mehreren seiner Äste befallen war. Da diese Variante des Herpes Zoster besonders mit Komplikationen behaftet sein kann, wird eine stationäre Behandlung empfohlen (Gross et al. 2003). Im Gegensatz hierzu zeigen Studien, dass bei ambulant behandelten Patienten der Nervus trigeminus nur in $5 \%$ bis $25 \%$ der Fälle befallen ist (Benbernou et al. 2011; Meister et al. 1999). Außerdem zeigen Daten aus einem französischen Hausarztnetzwerk, dass nur $1 \%$ der an Herpes Zoster erkrankten Patienten stationär behandelt werden (Gonzalez Chiappe et al. 2010). 


\section{Zusammenfassung}

Aus dem operativen Bereich ist bekannt, dass das Vorhandensein von chronischen Schmerzen mit einer erhöhten postoperativen Schmerzintensität assoziiert ist. Ziel dieser Studie ist es, den Einfluss von vorbestehenden chronischen Schmerzen auf akute Schmerzen bei Patienten mit akuter Herpes-Zoster-Infektion zu untersuchen.

Patienten, die aufgrund einer akuten Herpes-Zoster-Infektion stationär zur Therapie aufgenommen wurden, wurden hinsichtlich ihrer Schmerzgeschichte charakterisiert und verglichen. Dabei wurden Schmerzintensität, schmerzbedingte Funktionseinschränkungen, Analgetikakonsum, psychologischer Distress und die Druckschmerzschwelle als Maß der Schmerzempfindlichkeit an Tag 1, 4 und 7 des stationären Aufenthaltes und am Entlassungstag erhoben. Nach der Entlassung erfolgte nach drei und sechs Monaten eine telefonische Nachbefragung zu o. g. Parametern.

59 Patienten wurden in die Studie eingeschlossen. 25 Patienten (42\%) hatten vorbestehende chronische Schmerzen. Diese Patienten hatten im Rahmen ihrer akuten Herpes-Zoster-Infektion eine erhöhte Schmerzintensität an allen erhobenen Tagen während des stationären Aufenthaltes, zeigten mehr funktionelle Einschränkungen und mehr Nebenwirkungen, die mit der Schmerzbehandlung assoziiert sein könnten. Ein signifikanter Unterschied im Analgetikakonsum zeigte sich nicht. Die Patienten mit vorbestehenden chronischen Schmerzen wiesen eine längere stationäre Liegezeit auf. Bei Patienten ohne chronische Schmerzen korrelierte die zosterbedingte Schmerzintensität nur mit der Menge der eingenommenen Analgetika und keinen weiteren Faktoren. Im Gegensatz hierzu waren bei den Patienten mit chronischen Schmerzen die körperliche Gesundheit und das Vorhandensein von neuropathischen Schmerzcharakteristika mit der Intensität der Zosterschmerzen assoziiert. Es zeigte sich in der Nachbefragung, dass Patienten, die eine Post-Zoster-Neuralgie (PZN) entwickelten, eine geringere körperliche und psychische Gesundheit hatten und häufiger eine neuropathische Schmerzcharakteristik aufwiesen. Auch konsumierten PZN-Patienten höhere Mengen an Analgetika. Die Intensität der PZN war assoziiert mit der Dauer der stationären Behandlung und der maximalen Intensität der Zosterschmerzen am Entlassungstag.

Patienten mit vorbestehenden chronischen Schmerzen hatten eine erhöhte Intensität der Zosterschmerzen. Außerdem hatten sie vermehrt schmerzbezogene funktionelle Einschränkungen und wiesen eine längere stationäre Liegezeit auf. Diese Ergebnisse zeigten sich auch in Studien bei Patienten aus dem operativen Bereich. Da in dieser Studie stationär behandelte 
Patienten betrachtet wurden, sind die Ergebnisse nur eingeschränkt übertagbar auf ambulant behandelte Patienten mit einer akuten Herpes-Zoster-Infektion. 


\section{Literatur}

Allegri M, Casale R, di Matteo M, Minella C, Fanelli G (2014): Reduction of painful area as new possible therapeutic target in post-herpetic neuropathic pain treated with 5\% lidocaine medicated plaster: a case series. J Pain Res $\underline{7}$, 353-357

Althaus A, Hinrichs-Rocker A, Chapman R, Becker OA, Lefering R, Simanski C, Weber F, Moser K-H, Joppich R, Trojan S, et al. (2012): Development of a risk index for the prediction of chronic post-surgical pain: Chronic post-surgical pain. Eur J Pain $\underline{16}$, 901-910

Antonopoulou MD, Alegakis AK, Hadjipavlou AG, Lionis CD (2009): Studying the association between musculoskeletal disorders, quality of life and mental health. A primary care pilot study in rural Crete, Greece. BMC Musculoskelet Disord 10, 143-151

Arnow BA, Hunkeler EM, Blasey CM, Lee J, Constantino MJ, Fireman B, Kraemer HC, Dea R, Robinson R, Hayward C (2006): Comorbid depression, chronic pain, and disability in primary care. Psychosom Med $\underline{68}, 262-268$

Barman D (2015): Association Between Depression and Acute Pain in Adults Attending a Tertiary Care Hospital in Bhubaneswar. J Clin Diagn Res $\underline{9}, 8-11$

Baron R (2000): Neuropathische Schmerzen-Der lange Weg vom Mechanismus zur mechanismenorientierten Therapie. Anaesthesist 49, 373-386

Baron R (2006): CME: Diagnosis and treatment of neuropathic pain. Dtsch Arztebl 103, $2720-2730$

Benbernou A, Drolet M, Levin MJ, Schmader KE, Oxman MN, Johnson R, Patrick D, Camden S, Mansi JA, Brisson M (2011): Association between prodromal pain and the severity of acute herpes zoster and utilization of health care resources. Eur J Pain $\underline{15}, 1100-1106$

Bennett MI, Attal N, Backonja MM, Baron R, Bouhassira D, Freynhagen R, Scholz J, Tölle TR, Wittchen H-U, Jensen TS (2007): Using screening tools to identify neuropathic pain. Pain 127, 199-203

Bierke S, Häner M, Petersen W (2016): Influence of somatization and depressive symptoms on the course of pain within the first year after uncomplicated total knee replacement: a prospective study. Int Orthop $\underline{40}, 1353-1360$

Binder A, Baron R (2016): The Pharmacological Therapy of Chronic Neuropathic Pain. Dtsch Arzteblatt Int 113, 616-625

Birket-Smith M (2001): Somatization and chronic pain. Acta Anaesthesiol Scand 45, 1114 1120 
Bouhassira D, Chassany O, Gaillat J, Hanslik T, Launay O, Mann C, Rabaud C, Rogeaux O, Strady C (2012): Patient perspective on herpes zoster and its complications: An observational prospective study in patients aged over 50years in general practice. Pain $\underline{153}, 342-349$

Breivik H, Collett B, Ventafridda V, Cohen R, Gallacher D (2006): Survey of chronic pain in Europe: Prevalence, impact on daily life, and treatment. Eur J Pain 10, 287-287

Brown TA, Chorpita BF, Korotitsch W, Barlow DH (1997): Psychometric properties of the Depression Anxiety Stress Scales (DASS) in clinical samples. Behav Res Ther $\underline{35}$, 79-89

Capdevila X, Barthelet Y, Biboulet P, Ryckwaert Y, Rubenovitch J, d'Athis F (1999): Effects of perioperative analgesic technique on the surgical outcome and duration of rehabilitation after major knee surgery. Anesthesiology $\underline{91}, 8-15$

Casser H-R, Nagel B (2016): Qualität der Schmerztherapie in Deutschland - Multimodale Schmerztherapie: Struktur und Qualität. Anasthesiol Intensivmed Notfallmed Schmerzther 51, 56-67

Colloca L, Ludman T, Bouhassira D, Baron R, Dickenson AH, Yarnitsky D, Freeman R, Truini A, Attal N, Finnerup NB, et al. (2017): Neuropathic pain. Nat Rev Dis Primer $\underline{3}, 17002$

Courtney CA, Duffy K, Serpell MG, O’Dwyer PJ (2002): Outcome of patients with severe chronic pain following repair of groin hernia. Br J Surg $\underline{89}, 1310-1314$

Drolet M, Brisson M, Schmader KE, Levin MJ, Johnson R, Oxman MN, Patrick D, Blanchette C, Mansi JA (2010): The impact of herpes zoster and postherpetic neuralgia on health-related quality of life: a prospective study. Can Med Assoc J 182, 17311736

Dworkin RH, Nagasako EM, Johnson RW, Griffin DR (2001): Acute pain in herpes zoster: the famciclovir database project. Pain $\underline{94}, 113-119$

Dworkin RH, Gnann JW, Oaklander AL, Raja SN, Schmader KE, Whitley RJ (2008): Diagnosis and assessment of pain associated with herpes zoster and postherpetic neuralgia. J Pain $\underline{9}, 37-44$

Dworkin RH, O’Connor AB, Audette J, Baron R, Gourlay GK, Haanpää ML, Kent JL, Krane EJ, LeBel AA, Levy RM, et al. (2010): Recommendations for the Pharmacological Management of Neuropathic Pain: An Overview and Literature Update. Mayo Clin Proc $\underline{85}, 3-14$

Dworkin RH, Bruehl S, Fillingim RB, Loeser JD, Terman GW, Turk DC (2016): Multidimensional Diagnostic Criteria for Chronic Pain: Introduction to the ACTTIONAmerican Pain Society Pain Taxonomy (AAPT). J Pain 17, 1-9

Edelsberg JS, Lord C, Oster G (2011): Systematic review and meta-analysis of efficacy, safety, and tolerability data from randomized controlled trials of drugs used to treat postherpetic neuralgia. Ann Pharmacother 45, 1483-1490 
Erlenwein J, Stamer U, Koschwitz R, Koppert W, Quintel M, Meißner W, Petzke F (2014): Akutschmerztherapie in der stationären Versorgung an deutschen Krankenhäusern: Ergebnisse des Akutschmerzzensus 2012. Schmerz 28, 147-156

Erlenwein J, Przemeck M, Degenhart A, Budde S, Falla D, Quintel M, Pfingsten M, Petzke F (2016): The Influence of Chronic Pain on Postoperative Pain and Function After Hip Surgery: A Prospective Observational Cohort Study. J Pain 17, 236-247

Erlenwein J, Petzke F, Stamer U, Meißner W, Nauck F, Pogatzki-Zahn E, Koppert W, Maier C (2017): Rolle der Anästhesiologie in der schmerzmedizinischen und palliativmedizinischen Versorgung in deutschen Krankenhäusern. Anaesthesist 66, 579-588

Fayaz A, Croft P, Langford RM, Donaldson LJ, Jones GT (2016): Prevalence of chronic pain in the UK: a systematic review and meta-analysis of population studies. BMJ Open $\underline{6}$, e010364

Forbes HJ, Thomas SL, Smeeth L, Clayton T, Farmer R, Bhaskaran K, Langan SM (2016a): A systematic review and meta-analysis of risk factors for postherpetic neuralgia: PAIN $\underline{157}, 30-54$

Forbes HJ, Bhaskaran K, Thomas SL, Smeeth L, Clayton T, Mansfield K, Minassian C, Langan SM (2016b): Quantification of risk factors for postherpetic neuralgia in herpes zoster patients: A cohort study. Neurology $\underline{87}, 94-102$

Frettlöh J, Maier C, Gockel H, Zenz M, Hüppe M (2009): Patientenkollektiv deutscher schmerztherapeutischer Einrichtungen: Kerndaten von mehr als 10.000 Patienten. Schmerz 23, 576-591

Freynhagen R, Baron R, Gockel U, Tölle TR (2006): pain DETECT : a new screening questionnaire to identify neuropathic components in patients with back pain. Curr Med Res Opin 22, 1911-1920

Gerbershagen HJ, Dagtekin O, Rothe T, Heidenreich A, Gerbershagen K, Sabatowski R, Petzke F, Özgür E (2009): Risk factors for acute and chronic postoperative pain in patients with benign and malignant renal disease after nephrectomy. Eur J Pain $\underline{13}$, $853-860$

Gerbershagen HJ, Pogatzki-Zahn E, Aduckathil S, Peelen LM, Kappen TH, van Wijck AJM, Kalkman CJ, Meissner W (2014): Procedure-specific risk factor analysis for the development of severe postoperative pain. Anesthesiology 120, 1237-1245

Geurts JW, Willems PC, Kallewaard J-W, van Kleef M, Dirksen C (2018): The Impact of Chronic Discogenic Low Back Pain: Costs and Patients' Burden. Pain Res Manag $\underline{2018}, 4696180$

Giesecke T, Gracely RH, Grant MAB, Nachemson A, Petzke F, Williams DA, Clauw DJ (2004): Evidence of augmented central pain processing in idiopathic chronic low back pain. Arthritis Rheum 50, 613-623

Gilden DH, Kleinschmidt-DeMasters BK, LaGuardia JJ, Mahalingam R, Cohrs RJ (2000): Neurologic complications of the reactivation of varicella-zoster virus. N Engl J Med $\underline{342}, 635-645$ 
Gonzalez Chiappe S, Sarazin M, Turbelin C, Lasserre A, Pelat C, Bonmarin I, Chosidow O, Blanchon T, Hanslik T (2010): Herpes zoster: Burden of disease in France. Vaccine $\underline{28}, 7933-7938$

Goßrau G (2014): Postzosterneuralgie. Schmerz 28, 93-104

Gross G, Schöfer H, Wassilew S, Friese K, Timm A, Guthoff R, Pau HW, Malin JP, Wutzler P, Doerr HW (2003): Herpes zoster guideline of the German Dermatology Society (DDG). J Clin Virol 26, 277-289

Hapidou EG, O’Brien MA, Pierrynowski MR, de las Heras E, Patel M, Patla T (2012): Fear and Avoidance of Movement in People with Chronic Pain: Psychometric Properties of the 11-Item Tampa Scale for Kinesiophobia (TSK-11). Physiother Can 64, 235241

Haroutiunian S, Nikolajsen L, Finnerup NB, Jensen TS (2013): The neuropathic component in persistent postsurgical pain: a systematic literature review. Pain $\underline{154}, 95-102$

Hasenbring M: The Kiel Pain Inventory-Manual. Three questionnaire scales for assessment of pain-related cognitions, emotions and copying strategies. Huber, Bern 1994

Hasenbring M, Hallner D, Klasen B (2001): Psychologische Mechanismen im Prozess der Schmerzchronifizierung: Unter- oder überbewertet? Schmerz 15, 442-447

Häuser W, Schmutzer G, Henningsen P, Brähler E (2014): Chronische Schmerzen, Schmerzkrankheit und Zufriedenheit der Betroffenen mit der Schmerzbehandlung in Deutschland. Schmerz 28, 483-492

Häuser W, Schmutzer G, Hilbert A, Brähler E, Henningsen P (2015): Prevalence of Chronic Disabling Noncancer Pain and Associated Demographic and Medical Variables: A Cross-Sectional Survey in the General German Population. Clin J Pain $\underline{31}$, 886-892

Hildebrandt J, Pfingsten M, Maier C, Klinger R, Hasenbring M (1992): Zum Problem der Klassifikation chronischer Schmerzsyndrome: Multiaxiale Schmerzklassifikation MAS. Anasthesiol Intensivmed Notfallmed Schmerzther 27, 366-373

Hinrichs-Rocker A, Schulz K, Järvinen I, Lefering R, Simanski C, Neugebauer EAM (2009): Psychosocial predictors and correlates for chronic post-surgical pain (CPSP) - A systematic review. Eur J Pain 13, 719-730

Holmes A, Christelis N, Arnold C (2013): Depression and chronic pain. Med J Aust 199, $17-20$

Houben RMA, Leeuw M, Vlaeyen JWS, Goubert L, Picavet HSJ (2005): Fear of Movement/Injury in the General Population: Factor Structure and Psychometric Properties of an Adapted Version of the Tampa Scale for Kinesiophobia. J Behav Med 28, 415424

Hsu Y-W, Somma J, Hung Y-C, Tsai P-S, Yang C-H, Chen C-C (2005): Predicting postoperative pain by preoperative pressure pain assessment. Anesthesiology 103, 613618 
Ip HYV, Abrishami A, Peng PW, Wong J, Chung F (2009): Predictors of postoperative pain and analgesic consumption: a qualitative systematic review. Anesthesiology $\underline{111}$, $657-677$

Jenkinson C, Layte R, Jenkinson D, Lawrence K, Petersen S, Paice C, Stradling J (1997): A shorter form health survey: can the SF-12 replicate results from the SF-36 in longitudinal studies? J Public Health $\underline{19}$, 179-186

Johnson RW (2007): Zoster-associated pain: what is known, who is at risk and how can it be managed? Herpes $\underline{14}, 30-34$

Johnson RW, Bouhassira D, Kassianos G, Leplège A, Schmader KE, Weinke T (2010): The impact of herpes zoster and post-herpetic neuralgia on quality-of-life. BMC Med $\underline{8}$, $37-50$

Jung BF, Ahrendt GM, Oaklander AL, Dworkin RH (2003): Neuropathic pain following breast cancer surgery: proposed classification and research update. Pain $\underline{104}, 1-13$

Jung BF, Johnson RW, Griffin DRJ, Dworkin RH (2004): Risk factors for postherpetic neuralgia in patients with herpes zoster. Neurology $\underline{62}, 1545-1551$

Karaman S, Karaman T, Dogru S, Onder Y, Citil R, Bulut YE, Tapar H, Sahin A, Arici S, Kaya Z, others (2014): Prevalence of sleep disturbance in chronic pain. Eur Rev Med Pharmacol Sci $\underline{18}, 2475-2481$

Katz J, Seltzer Z (2009): Transition from acute to chronic postsurgical pain: risk factors and protective factors. Expert Rev Neurother $\underline{9}, 723-744$

Kehlet H (2004): Effect of postoperative pain treatment on outcome? Current status and future strategies. Langenbecks Arch Surg 389, 244-249

Kehlet H, Jensen TS, Woolf CJ (2006): Persistent postsurgical pain: risk factors and prevention. Lancet $\underline{367}, 1618-1625$

Kiosses D, Ravdin L, Stern A, Bolier R, Kenien C, Reid M (2017): Problem Adaptation Therapy for Pain (PATH-Pain): A Psychosocial Intervention for Older Adults with Chronic Pain and Negative Emotions in Primary Care. Geriatrics 2, $5-14$

Korczak D, Kuczera C, Rust M (2013): Acute pain treatment on postoperative and medical non-surgical wards. GMS Health Technol Assess $\underline{9}, 5$

Koroschetz J, Rehm SE, Gockel U, Brosz M, Freynhagen R, Tölle TR, Baron R (2011): Fibromyalgia and neuropathic pain--differences and similarities. A comparison of 3057 patients with diabetic painful neuropathy and fibromyalgia. BMC Neurol $\underline{11}$, 55-63

Kost RG, Straus SE (1996): Postherpetic Neuralgia - Pathogenesis, Treatment, and Prevention. N Engl J Med 335, 32-42

Kroenke K, Spitzer RL, Williams JBW (2002): The PHQ-15: validity of a new measure for evaluating the severity of somatic symptoms. Psychosom Med $\underline{64}, 258-266$ 
Kurita GP, Sjøgren P, Juel K, Højsted J, Ekholm O (2012): The burden of chronic pain: A cross-sectional survey focussing on diseases, immigration, and opioid use. Pain $\underline{153}$, $2332-2338$

Lapolla W (2011): Incidence of Postherpetic Neuralgia After Combination Treatment With Gabapentin and Valacyclovir in Patients With Acute Herpes Zoster: Open-label Study. Arch Dermatol 147, 901-907

Lee S, Chen PP, Lee A, Ma M, Wong CM, Gin T (2005): A prospective evaluation of healthrelated quality of life in Hong Kong Chinese patients with chronic non-cancer pain. Hong Kong Med J 11, 174-180

Lovibond PF, Lovibond SH (1995): The structure of negative emotional states: Comparison of the Depression Anxiety Stress Scales (DASS) with the Beck Depression and Anxiety Inventories. Behav Res Ther $\underline{33}$, 335-343

Løyland B, Miaskowski C, Paul SM, Dahl E, Rustøen T (2010): The relationship between chronic pain and health-related quality of life in long-term social assistance recipients in Norway. Qual Life Res $\underline{19}, 1457-1465$

Maier C, Nestler N, Richter H, Hardinghaus W, Pogatzki-Zahn E, Zenz M, Osterbrink J (2010): The quality of pain management in German hospitals. Dtsch Ärztebl Int 107, 607-614

Marchettini P, Lacerenza M, Mauri E, Marangoni C (2006): Painful peripheral neuropathies. Curr Neuropharmacol $\underline{4}, 175-181$

McWilliams LA, Cox BJ, Enns MW (2003): Mood and anxiety disorders associated with chronic pain: an examination in a nationally representative sample. Pain $\underline{106}, 127-$ 133

Meissner W, Mescha S, Rothaug J, Zwacka S, Goettermann A, Ulrich K, Schleppers A (2008): Quality improvement in postoperative pain management. Dtsch Ärzeblatt Int $\underline{105}, 865-870$

Meister W, Neiss A, Gross G, Doerr H, Höbel W, Malin P, von Essen J, Reimann B, Witke C, Wutzler P (1998a): A prognostic score for postherpetic neuralgia in ambulatory patients. Infection $\underline{26}, 359-363$

Meister W, Neiss A, Gross G, Doerr H, Höbel W, Malin J, von Essen J, Reimann B, Witke C, Wutzler P (1998b): Demography, symptomatology, and course of disease in ambulatory zoster patients. A physician-based survey in Germany. Intervirology $\underline{41}$, 272-277

Meister W, Neiss A, Gross G, Doerr H-W, Höbel W, Malin J-P, von Essen J, Reimann BY, Witke C, Wutzler P (1999): Demography, symptomatology, and course of disease in ambulatory zoster patients. Intervirology $41,272-277$

Melotti RM, Samolsky-Dekel BG, Ricchi E, Chiari P, Giacinto I, Carosi F, Nino G (2005): Pain prevalence and predictors among inpatients in a major Italian teaching hospital. A baseline survey towards a pain free hospital. Eur J Pain $\underline{9}, 485-485$ 
Merskey H, Albe Fessard D, Bonica JJ, Carmon A, Dubner R, Kerr FWL, Lindblom U Mumford JM, Nathan PW, Noordenbos W et al. (1979): Pain terms: a list with definitions and notes on usage. Recommended by the IASP subcommittee on taxonomy. Pain $\underline{6}, 249-252$

Mikkelsen T, Werner MU, Lassen B, Kehlet H (2004): Pain and sensory dysfunction 6 to 12 months after inguinal herniotomy. Anesth Analg 99, 146-151

Nagel B, Gerbershagen HU, Lindena G, Pfingsten M (2002): Entwicklung und empirische Überprüfung des Deutschen Schmerzfragebogens der DGSS. Schmerz 16, 263-270

Nicholas MK, Coulston CM, Asghari A, Malhi GS (2009): Depressive symptoms in patients with chronic pain. Med J Aust $\underline{190}$, 66-70

Nickel R, Raspe HH (2001): Chronischer Schmerz: Epidemiologie und Inanspruchnahme. Nervenarzt $\underline{72}, 897-906$

Nilges P, Essau C (2015): Die Depressions-Angst-Stress-Skalen. Schmerz Berl Ger 29, 649657

Noiseux NO, Callaghan JJ, Clark CR, Zimmerman MB, Sluka KA, Rakel BA (2014): Preoperative Predictors of Pain Following Total Knee Arthroplasty. J Arthroplasty 29, $1383-1387$

Oaklander AL (2008): Mechanisms of pain and itch caused by herpes zoster (shingles). J Pain $\underline{9}, 10-18$

O'Connor AB (2009): Neuropathic pain: quality-of-life impact, costs and cost effectiveness of therapy. Pharmacoeconomics $\underline{27}, 95-112$

Oosterwijck JV, Nijs J, Meeus M, Paul L (2013): Evidence for central sensitization in chronic whiplash: A systematic literature review. Eur J Pain 17, 299-312

Oxman MN, Levin Mj, Johnson GR, Schmader KE, Straus SE, Gelb LD, Arbeit RD, Simberkoff MS, Gershon AA, Davis LE, others (2005): A vaccine to prevent herpes zoster and postherpetic neuralgia in older adults. N Engl J Med 352, 2271-2284

Pan PH, Coghill R, Houle TT, Seid MH, Lindel WM, Parker RL, Washburn SA, Harris L, Eisenach JC (2006): Multifactorial preoperative predictors for postcesarean section pain and analgesic requirement. Anesthesiology 104, 417-425

Pergolizzi J, Ahlbeck K, Aldington D, Alon E, Coluzzi F, Dahan A, Huygen F, KocotKępska M, Mangas AC, Mavrocordatos P, et al. (2013): The development of chronic pain: physiological CHANGE necessitates a multidisciplinary approach to treatment. Curr Med Res Opin 29, 1127-1135

Peters ML, Sommer M, de Rijke JM, Kessels F, Heineman E, Patijn J, Marcus MAE, Vlaeyen JWS, van Kleef M (2007): Somatic and Psychologic Predictors of Longterm Unfavorable Outcome After Surgical Intervention: Ann Surg 245, 487-494

Petzke F, Clauw DJ, Ambrose K, Khine A, Gracely RH (2003): Increased pain sensitivity in fibromyalgia: effects of stimulus type and mode of presentation. Pain $\underline{105}$, 403-413 
Pogatzki-Zahn E, Englbrecht J, Schug S (2009): Acute pain management in patients with fibromyalgia and other diffuse chronic pain syndromes. Curr Opin Anaesthesiol $\underline{22}$, $627-633$

Poobalan AS, Bruce J, King PM, Chambers WA, Krukowski ZH, Smith WC (2001): Chronic pain and quality of life following open inguinal hernia repair. Br J Surg $\underline{88}$, 11221126

Rehm SE, Koroschetz J, Gockel U, Brosz M, Freynhagen R, Tolle TR, Baron R (2010): A cross-sectional survey of 3035 patients with fibromyalgia: subgroups of patients with typical comorbidities and sensory symptom profiles. Rheumatology $\underline{49}, 1146-1152$

Roelofs J, Goubert L, Peters ML, Vlaeyen JWS, Crombez G (2004): The Tampa Scale for Kinesiophobia: further examination of psychometric properties in patients with chronic low back pain and fibromyalgia. Eur J Pain $\underline{8}, 495-502$

Schaible H-G (2007): Pathophysiologie des Schmerzes. Orthopäde $\underline{36}$, 8-16

Schmader KE, Sloane R, Pieper C, Coplan PM, Nikas A, Saddier P, Chan ISF, Choo P, Levin MJ, Johnson G, et al. (2007): The impact of acute herpes zoster pain and discomfort on functional status and quality of life in older adults. Clin J Pain $\underline{23}, 490$ 496

Schnabel A, Pogatzki-Zahn E (2010): Prädiktoren für chronische Schmerzen nach Operationen: Was wissen wir wirklich? Schmerz 24, 517-533

Schmitt N, Gerbershagen HU (1990): The Mainz pain staging system (MPSS) for chronic pain. Pain $\underline{5}, 484$

Scott FT, Leedham-Green ME, Barrett-Muir WY, Hawrami K, Gallagher WJ, Johnson R, Breuer J (2003): A study of shingles and the development of postherpetic neuralgia in East London. J Med Virol 70, 24-30

Siedler A, Koch J, Garbe E, Hengel H, von Kries R, Ledig T, Mertens T, Zepp F, Überla K (2019): Background paper to the decision to recommend the vaccination with the inactivated herpes zoster subunit vaccine. Bundesgesundheitsblatt Gesundheitsforschung Gesundheitsschutz $\underline{62}$, 352-376

Solomon CG, Johnson RW, Rice ASC (2014): Postherpetic Neuralgia. N Engl J Med $\underline{371}$, $1526-1533$

Song H, Lee J, Lee M, Choi WS, Choi JH, Lee MS, Hashemi M, Rampakakis E, Kawai K, White R, et al. (2014): Burden of illness, quality of life, and healthcare utilization among patients with herpes zoster in South Korea: a prospective clinical-epidemiological study. Int J Infect Dis $\underline{20}, 23-30$

Stormo KJ, Kee WG, Steedham S, Middaugh S (1998): Medication quantification scores and evaluation of patient pharmacology profiles. Curr Rev Pain $\underline{2}, 171-174$

Tang NK, Wright KJ, Salkovskis PM (2007): Prevalence and correlates of clinical insomnia co-occurring with chronic back pain. J Sleep Res $\underline{16}$, 85-95 
Taylor R, Lovibond PF, Nicholas MK, Cayley C, Wilson PH (2005): The utility of somatic items in the assessment of depression in patients with chronic pain: a comparison of the Zung Self-Rating Depression Scale and the Depression Anxiety Stress Scales in chronic pain and clinical and community samples. Clin J Pain 21, 91-100

Treede R-D, Jensen TS, Campbell JN, Cruccu G, Dostrovsky JO, Griffin JW, Hansson P, Hughes R, Nurmikko T, Serra J (2008): Neuropathic pain: redefinition and a grading system for clinical and research purposes. Neurology $\underline{70}$, 1630-1635

Ultsch B, Siedler A, Rieck T, Reinhold T, Krause G, Wichmann O (2011): Herpes zoster in Germany: quantifying the burden of disease. BMC Infect Dis $\underline{11}$, 173-182

van Hecke O, Austin SK, Khan RA, Smith BH, Torrance N (2014): Neuropathic pain in the general population: a systematic review of epidemiological studies. Pain $\underline{155}, 654$ 662

Von Korff M, Ormel J, Keefe FJ, Dworkin SF (1992): Grading the severity of chronic pain. Pain $\underline{50}, 133-149$

Wareham DW, Breuer J (2007): Herpes zoster. BMJ 334, 1211-1215

Werner RN, Nikkels AF, Marinović B, Schäfer M, Czarnecka-Operacz M, Agius AM, BataCsörgő Z, Breuer J, Girolomoni G, Gross GE, et al. (2017a): European consensusbased (S2k) Guideline on the Management of Herpes Zoster - guided by the European Dermatology Forum (EDF) in cooperation with the European Academy of Dermatology and Venereology (EADV), Part 1: Diagnosis. J Eur Acad Dermatol Venereol $\underline{31}, 9-19$

Werner RN, Nikkels AF, Marinović B, Schäfer M, Czarnecka-Operacz M, Agius AM, BataCsörgő Z, Breuer J, Girolomoni G, Gross GE, et al. (2017b): European consensusbased (S2k) Guideline on the Management of Herpes Zoster - guided by the European Dermatology Forum (EDF) in cooperation with the European Academy of Dermatology and Venereology (EADV), Part 2: Treatment. J Eur Acad Dermatol Venereol $\underline{31}, 20-29$

Whitley RJ, Shukla S, Crooks RJ (1998): The identification of risk factors associated with persistent pain following herpes zoster. J Infect Dis $\underline{178}, 71-75$

Wong WS, Fielding R (2011): Prevalence and characteristics of chronic pain in the general population of Hong Kong. J Pain 12, 236-245

Yawn BP, Gilden D (2013): The global epidemiology of herpes zoster. Neurology $\underline{81}, 928$ 930

Zimmermann M (2004): Der Chronische Schmerz. Orthopäde 33, 508-514 


\section{Danksagung}

Als erstes möchte ich mich bei den Patienten dieser Studie bedanken. Ohne sie wäre diese Studie nicht möglich gewesen. Die Patienten wurden inklusive Nachbefragung meist sechsmal kontaktiert und haben weit über fünf Stunden Zeit für diese Studie aufgebracht.

Außerdem möchte ich mich bei dem Team der Stationen 6024 und 6023 der Universitätsmedizin Göttingen für die Unterstützung bedanken. Mein besonderer Dank gilt dabei den Case-Managerinnen Doris Trunk und Marion Buschemöhle, die mich informiert haben, sobald ein Patienten mir Herpes Zoster stationär aufgenommen wurde.

Bei Johanna Rudack und Carina Schröder aus der Schmerzambulanz möchte ich mich für die Einarbeitung in die Studie bedanken. Ebenso gilt mein Dank Dr. Müller und Professor Dr. Pfingsten aus der Schmerzambulanz, die mir bei der Auswertung der Fragemodule stets mit Rat zur Seite standen.

Ganz besonderer Dank gilt meinem Doktorvater Herrn Professor Dr. Petzke und meinem Betreuer Herrn PD Dr. Erlenwein aus der Schmerzambulanz. Sie waren immer für mich erreichbar und haben mich bei der Entstehung dieser Studie bestens unterstützt. 